REPRESENTATION THEORY

An Electronic Journal of the American Mathematical Society

Volume 10, Pages 353-379 (August 17, 2006)

S $1088-4165(06) 00315-3$

\title{
CHARACTER SHEAVES ON DISCONNECTED GROUPS, IX
}

\author{
G. LUSZTIG
}

\begin{abstract}
We associate a two-sided cell to any (parabolic) character sheaf. We study the interaction between the duality operator for character sheaves
\end{abstract} and the operation of "twisted induction".

\section{INTRODUCTION}

Throughout this paper, $G$ denotes a fixed, not necessarily connected, reductive algebraic group over an algebraically closed field $\mathbf{k}$. This paper is a part of a series L9 which attempts to develop a theory of character sheaves on $G$.

One of the main constructions in L3] (going back to L14]) was a procedure which to any character sheaf on $G^{0}$ associates a certain two-sided cell in an (extended) Coxeter group. A variant of this construction (restricted to "unipotent" character sheaves) was later given by Grojnowski Gr. Here we give a construction which generalizes that in [L3] (and takes into account the approach in $\mathrm{Gr}$ ) which to any (parabolic) character sheaf on $Z_{J, D}$ associates a certain type of two-sided cell.

The paper is organized as follows. In Section 40 we study certain equivariant sheaves on $G^{0} / U^{*} \times G^{0} / U^{*}$ (where $U^{*}$ is the unipotent radical of a Borel in $G^{0}$ ) under the convolution operation. Some results in this section are implicit in L14, Ch.1]. In Section 41 we study the character sheaves on $Z_{\emptyset, D}$ (where $D$ is a connected component of $G$ ) by connecting them with sheaves on $G^{0} / U^{*} \times G^{0} / U^{*}$. We use this study to attach a two-sided cell to any character sheaf on $Z_{J, D}$. (See 41.4.) In Section 42 we study the interaction between the duality operation $\mathbf{d}$ (see 38.10 , 38.11 ) and the functor $\mathfrak{f}_{\emptyset, \mathbf{I}}$ (see 36.4). The main result in this section is Proposition 42.9 which contains [L3, III, Cor. 15.8(b)] as a special case (with $G=G^{0}, v=1$ ).

Notation. We fix a 1-dimensional $\overline{\mathbf{Q}}_{l}$-vector space $V$ with a given isomorphism $V^{\otimes 2} \stackrel{\sim}{\longrightarrow} \overline{\mathbf{Q}}_{l}(1)$ (Tate twist of $\overline{\mathbf{Q}}_{l}$ ). For $n \in \mathbf{N}$ we set $\overline{\mathbf{Q}}_{l}(n / 2)=V^{\otimes n}$. For $n \in \mathbf{Z}, n<0$ let $\overline{\mathbf{Q}}_{l}(n / 2)$ be the dual space of $\overline{\mathbf{Q}}_{l}(-n / 2)$. If $X$ is an algebraic variety and $A \in \mathcal{D}(X), n \in \mathbf{Z}$, we write $A(n / 2)$ instead of $A \otimes \overline{\mathbf{Q}}_{l}(n / 2)$ and $A[[n / 2]]$ instead of $A[n](n / 2)$. (When $n$ is even, this agrees with the notation in [L9, II, p. 73].)

\section{Contents}

40. Sheaves on $G^{0} / U^{*} \times G^{0} / U^{*}$.

41. Character sheaves and two-sided cells.

42. Duality and the functor $\mathfrak{f}_{\emptyset, \mathbf{I}}$.

Received by the editors February 8, 2006.

2000 Mathematics Subject Classification. Primary 20G99.

Supported in part by the National Science Foundation.

(C)2006 American Mathematical Society 353

Reverts to public domain 28 years from publication 


\section{SHEAVES ON $G^{0} / U^{*} \times G^{0} / U^{*}$}

40.1. Let $\mathcal{A}=\mathbf{Z}\left[v, v^{-1}\right]$. Let $\hat{H}$ (resp. $H$ ) be the $\mathcal{A}$-module consisting of all formal (resp. finite) linear combinations $\sum_{w \in \mathbf{W}, \lambda \in \underline{\mathfrak{s}}} a_{w, \lambda} \tilde{T}_{w} 1_{\lambda}$ with $a_{w, \lambda} \in \mathcal{A}$. Note that $H$ is naturally an $\mathcal{A}$-submodule of $\hat{H}$ with $\mathcal{A}$-basis $\left\{\tilde{T}_{w} 1_{\lambda} ; w \in \mathbf{W}, \lambda \in \underline{\mathfrak{s}}\right\}$. For any $n \in \mathbf{N}_{\mathbf{k}}^{*}$, the $\mathcal{A}$-submodule of $H$ spanned by $\left\{\tilde{T}_{w} 1_{\lambda} ; w \in \mathbf{W}, \lambda \in \underline{\mathfrak{s}}_{n}\right\}$ may be naturally identified with $H_{n}$ (see $31.2(\mathrm{a})$ ). There is a unique $\mathcal{A}$-algebra structure on $\hat{H}$ in which the product of two elements

$$
h=\sum_{w \in \mathbf{W}, \lambda \in \underline{\mathfrak{s}}} a_{w, \lambda} \tilde{T}_{w} 1_{\lambda}, h^{\prime}=\sum_{w^{\prime} \in \mathbf{W}, \lambda^{\prime} \in \underline{\mathfrak{s}}} a_{w^{\prime}, \lambda^{\prime}}^{\prime} \tilde{T}_{w^{\prime}} 1_{\lambda^{\prime}}
$$

as above is $h h^{\prime}=\sum_{y \in \mathbf{W}, \nu \in \underline{\mathfrak{s}}} b_{y^{\prime}, \nu} \tilde{T}_{y} 1_{\nu}$ where for any $\nu \in \underline{\mathfrak{s}}$,

$$
\sum_{w, w^{\prime} \in \mathbf{W}} a_{w, w^{\prime-1} \nu} a_{w^{\prime}, \nu}^{\prime} \tilde{T}_{w} \tilde{T}_{w^{\prime}} 1_{\nu}=\sum_{y \in \mathbf{W}} b_{y, \nu} \tilde{T}_{y} 1_{\nu}
$$

is computed in the algebra structure of $H_{n}$ for any $n$ such that $\nu \in \underline{\mathfrak{s}}_{n}$. Thus $\hat{H}$ becomes an associative algebra with $1 ; H$ is a subalgebra (without 1 ) and, for $n \in \mathbf{N}_{\mathbf{k}}^{*}, H_{n}$ is a subalgebra (with a different 1 ) with the algebra structure as in 31.2 .

Now in the definition of $\hat{H}$ given above, although $\tilde{T}_{w} 1_{\lambda}$ is defined, the elements $\tilde{T}_{w}, 1_{\lambda}$ are not defined separately (as was the case in $H_{n}$ ). To remedy this we set $\tilde{T}_{w}=\sum_{\lambda \in \underline{\mathfrak{s}}} \tilde{T}_{w} 1_{\lambda} \in \hat{H}$ (for $w \in \mathbf{W}$ ) and $1_{\lambda}=\tilde{T}_{1} 1_{\lambda} \in H$ (for $\lambda \in \underline{\mathfrak{s}}$ ). Then $\tilde{T}_{w} 1_{\lambda}$ is the product of $\tilde{T}_{w}, 1_{\lambda}$ in the algebra $\hat{H}$. Note that $\tilde{T}_{1}$ is the unit element of $\hat{H}$ and the following equalities hold in $\hat{H}$ :

$1_{\lambda} 1_{\lambda}=1_{\lambda}$ for $\lambda \in \underline{\mathfrak{s}}, 1_{\lambda} 1_{\lambda^{\prime}}=0$ for $\lambda \neq \lambda^{\prime}$ in $\underline{\mathfrak{s}}$;

$\tilde{T}_{w} \tilde{T}_{w^{\prime}}=\tilde{T}_{w w^{\prime}}$ for $w, w^{\prime} \in \mathbf{W}$ such that $l\left(w w^{\prime}\right)=l(w)+l\left(w^{\prime}\right)$;

$\tilde{T}_{w} 1_{\lambda}=1_{w \lambda} \tilde{T}_{w}$ for $w \in \mathbf{W}, \lambda \in \underline{\mathfrak{s}}$

$\tilde{T}_{s}^{2}=\tilde{T}_{1}+\left(v-v^{-1}\right) \sum_{\lambda \in \underline{\mathfrak{s}} ; s \in \mathbf{W}_{\lambda}} \tilde{T}_{s} 1_{\lambda}$ for $s \in \mathbf{I}$.

By a standard argument we see that

(a) $H$ is exactly the $\mathcal{A}$-algebra defined by the generators $\tilde{T}_{w} 1_{l}(w \in \mathbf{W}, \lambda \in \underline{\mathfrak{s}})$ and the relations:

$\left(\tilde{T}_{w} 1_{\lambda}\right)\left(\tilde{T}_{w^{\prime}} 1_{\lambda^{\prime}}\right)=0$ if $w, w^{\prime} \in \mathbf{W}, \lambda, \lambda^{\prime} \in \underline{\mathfrak{s}}, w^{\prime} \lambda^{\prime} \neq \lambda$,

$\left(\tilde{T}_{w} 1_{w^{\prime} \lambda^{\prime}}\left(\tilde{T}_{w^{\prime}} 1_{\lambda^{\prime}}\right)=\tilde{T}_{w w^{\prime}} 1_{\lambda^{\prime}}\right.$ if $w, w^{\prime} \in \mathbf{W}, \lambda, \lambda^{\prime} \in \underline{\mathfrak{s}}, l\left(w w^{\prime}\right)=l(w)+l\left(w^{\prime}\right)$,

$\left(\tilde{T}_{s} 1_{s \lambda^{\prime}}\right)\left(\tilde{T}_{s} 1_{\lambda^{\prime}}\right)=\tilde{T}_{1} 1_{\lambda^{\prime}}+\left(v-v^{-1}\right) c \tilde{T}_{s} 1_{\lambda^{\prime}}$ if $s \in \mathbf{I}, \lambda^{\prime} \in \underline{\mathfrak{s}}$ where $c=1$ for $s \in \mathbf{W}_{\lambda^{\prime}}$ and $c=0$ for $s \notin \mathbf{W}_{\lambda^{\prime}}$.

40.2. Let $R, R^{+}$be as in 28.3. The following result is well known:

(a) If $w \in \mathbf{W}, \alpha \in R^{+}$and $s_{\alpha}$ is as in 28.3, then we have $l\left(w s_{\alpha}\right)>l(w)$ if and only if $w(\alpha) \in R^{+}$.

Let $\lambda \in \underline{\mathfrak{s}}$. Let $R_{\lambda}, R_{\lambda}^{+}, \mathbf{W}_{\lambda}, H_{\lambda}$ be as in 34.2 . We write $\vee_{\lambda}$ instead of $\vee_{\lambda}^{D}$ (as in 34.4 with $D=G^{0}$ ). We show that

(b) if $w \in \mathbf{W}$, then $w \mathbf{W}_{\lambda}$ contains a unique element $w_{1}$ of minimal length; it is characterized by the property $w_{1}\left(R_{\lambda}^{+}\right) \subset R^{+}$.

Let $w_{1}$ be an element of minimal length in $w \mathbf{W}_{\lambda}$. Let $\alpha \in R_{\lambda}^{+}$. Then $l\left(w_{1} s_{\alpha}\right) \geq$ $l\left(w_{1}\right)$. Since $l\left(w_{1} s_{\alpha}\right)=l\left(w_{1}\right)+1 \bmod 2$ we see that $l\left(w_{1} s_{\alpha}\right)>l\left(w_{1}\right)$. By (a) we have $w_{1}(\alpha) \in R^{+}$. Thus, $w_{1}\left(R_{\lambda}^{+}\right) \subset R^{+}$. Now let $u \in \mathbf{W}_{\lambda}-\{1\}$. We pick $\alpha \in R_{\lambda}^{+}$ such that $u(\alpha)^{-1} \in R_{\lambda}^{+}$; then $w_{1} u(\alpha)^{-1} \in R^{+}$. If $w_{1} u$ has minimal length in $w \mathbf{W}_{\lambda}$, 
then by an earlier part of the argument applied to $w_{1} u$ instead of $w_{1}$ we have $w_{1} u(\alpha) \in R^{+}$, a contradiction. We see that $w_{1}$ is the unique element of minimal length in $w \mathbf{W}_{\lambda}$. It remains to show that if $u \in \mathbf{W}_{\lambda}$ satisfies $w_{1} u\left(R_{\lambda}^{+}\right) \subset R^{+}$, then $u=1$. If $u \neq 1$, then by an earlier part of the argument we have $w_{1} u(\alpha)^{-1} \in R^{+}$ for some $\alpha \in R_{\lambda}^{+}$, a contradiction. This proves (b).

We show that

(c) if $s \in \mathbf{I}$ and $w \in \mathbf{W}$ has minimal length in $w \mathbf{W}_{\lambda}$, then either (i) sw has minimal length in $s w \mathbf{W}_{\lambda}$ or (ii) $w^{-1} s w \in \mathbf{W}_{\lambda}$.

There is a unique $\beta \in R^{+}$such that $s(\beta)^{-1} \in R^{+}$. Assume that (i) does not hold. By (b) there exists $\alpha \in R_{\lambda}^{+}$such that $s w(\alpha)^{-1} \in R^{+}$; moreover, $w(\alpha) \in R^{+}$. Hence $w(\alpha)=\beta$. We have $w^{-1}(\beta)=\alpha \in R_{\lambda}$, hence $w^{-1} s w \in \mathbf{W}_{\lambda}$ and (ii) holds. This proves (c).

For $z \in \mathbf{W}_{\lambda}$ let $\tilde{T}_{z}^{\lambda}, c_{z}^{\lambda} \in H_{\lambda}$ be as in 34.2. Then $c_{z}^{\lambda}=\sum_{z^{\prime} \in \mathbf{W}_{\lambda}} p_{z^{\prime}, z}^{\lambda} \tilde{T}_{z^{\prime}}^{\lambda}$ where $p_{z^{\prime}, z}^{\lambda} \in \mathbf{Z}\left[v^{-1}\right]$ are uniquely defined.

For any $w \in \mathbf{W}, \lambda \in \underline{\mathfrak{s}}$ there is a unique element of $H$ which is equal to $c_{w, \lambda} \in H_{n}$ (see 34.4) for any $n$ such that $\lambda \in \underline{\mathfrak{s}}_{n}$; we denote this element again by $c_{w, \lambda}$. We have $c_{w, \lambda}=\sum_{w^{\prime} \in \mathbf{W}} \pi_{w^{\prime}, w, \lambda} \tilde{T}_{w^{\prime}} 1_{\lambda}$ where $\pi_{w^{\prime}, w, \lambda} \in \mathbf{Z}\left[v^{-1}\right]$ are uniquely defined. Note that $\left\{c_{w, \lambda} ; w \in \mathbf{W}, \lambda \in \underline{\mathfrak{s}}\right\}$ is an $\mathcal{A}$-basis of $H$.

Now:

(d) Let $w, w^{\prime} \in \mathbf{W}$. We write $w=w_{1} z, w^{\prime}=w_{1}^{\prime} z^{\prime}$ where $w_{1}$ has minimal length in $w \mathbf{W}_{\lambda}$, w $w_{1}^{\prime}$ has minimal length in $w^{\prime} \mathbf{W}_{\lambda}$ and $z, z^{\prime} \in \mathbf{W}_{\lambda}$. If $w_{1} \neq w_{1}^{\prime}$, then $\pi_{w^{\prime}, w, \lambda}=0$. If $w_{1}=w_{1}^{\prime}$, then $\pi_{w^{\prime}, w, \lambda}=p_{z^{\prime}, z}^{\lambda}$.

From the definitions we see that if $w \lambda \neq w^{\prime} \lambda$, then $\pi_{w^{\prime}, w, \lambda}=0$. Thus we may assume that $w \lambda=w^{\prime} \lambda$. We choose a sequence $s_{1}, s_{2}, \ldots, s_{r}$ in $\mathbf{I}$ such that $w \lambda=$ $w^{\prime} \lambda=s_{r} s_{r-1} \ldots s_{1} \lambda \neq s_{r-1} \ldots s_{1} \lambda \neq \ldots \neq s_{1} \lambda \neq \lambda$.

We show that for $k \in[0, r], s_{k} s_{k-1} \ldots s_{1}$ has minimal length in $s_{k} s_{k-1} \ldots s_{1} \mathbf{W}_{\lambda}$. We argue by induction. For $k=0$ the result is obvious. Assume now that $k \in$ $[1, r]$. Since $s_{k-1} \ldots s_{1}$ has minimal length in $s_{k-1} \ldots s_{1} \mathbf{W}_{\lambda}$ and $s_{k} s_{k-1} \ldots s_{1} \lambda \neq$ $s_{k-1} \ldots s_{1} \lambda$, we see from (c) that $s_{k} s_{k-1} \ldots s_{1}$ has minimal length in $s_{k} s_{k-1} \ldots s_{1} \mathbf{W}_{\lambda}$ as required.

In particular, $s_{r} s_{r-1} \ldots s_{1}$ has minimal length in $s_{r} s_{r-1} \ldots s_{1} \mathbf{W}_{\lambda}$. Since $w \lambda=$ $s_{r} s_{r-1} \ldots s_{1} \lambda$ we have $w=s_{r} s_{r-1} \ldots s_{1} h_{1} h_{2}$ where $h_{1} \in \vee_{\lambda}, h_{2} \in \mathbf{W}_{\lambda}$. Then both $w_{1}$ and $s_{r} s_{r-1} \ldots s_{1} h_{1}$ have minimal length in $s_{r} s_{r-1} \ldots s_{1} h_{1} \mathbf{W}_{\lambda}=w \mathbf{W}_{\lambda}=w_{1} \mathbf{W}_{\lambda}$; using (b) we deduce that $s_{r} s_{r-1} \ldots s_{1} h_{1}=w_{1}$. Hence $s_{1} \ldots s_{r} w=s_{1} \ldots s_{r} w_{1} z=$ $h_{1} z$. Similarly, $s_{1} \ldots s_{r} w^{\prime}=h_{1}^{\prime} z^{\prime}$ where $h_{1}^{\prime} \in \vee_{\lambda}$.

From the results in 34.7-34.10 we see that $\pi_{w^{\prime}, w, \lambda}=p_{s_{1} \ldots s_{r} w^{\prime}, s_{1} \ldots s_{r} w}^{\lambda}=p_{h_{1}^{\prime} z^{\prime}, h_{1} z}^{\lambda}$. Using $h_{1}, h_{1}^{\prime} \in V_{\lambda}$ and the definitions (34.2) we see that $p_{h_{1}^{\prime} z^{\prime}, h_{1} z}^{\lambda}=0$ if $h_{1} \neq h_{1}^{\prime}$ and $p_{h_{1}^{\prime} z^{\prime}, h_{1} z}^{\lambda}=p_{z^{\prime}, z}^{\lambda}$ if $h_{1}=h_{1}^{\prime}$.

It remains to show that we have $w_{1}=w_{1}^{\prime}$ if and only if $h_{1}=h_{1}^{\prime}$. We have $s_{r} s_{r-1} \ldots s_{1}=h_{1}^{-1} w_{1}$ and similarly $s_{r} s_{r-1} \ldots s_{1}=\left(h_{1}^{\prime}\right)^{-1} w_{1}^{\prime}$. Hence $h_{1}^{-1} w_{1}=$ $\left(h_{1}^{\prime}\right)^{-1} w_{1}^{\prime}$. We see that $w_{1}=w_{1}^{\prime}$ if and only if $h_{1}=h_{1}^{\prime}$. This proves $(\mathrm{d})$.

For $w^{\prime} \leq w$ in $\mathbf{W}, \lambda \in \underline{\mathfrak{s}}$ and $i \in \mathbf{Z}$ we define $N_{i, w^{\prime}, w, \lambda} \in \mathbf{Z}$ by

(e) $\pi_{w^{\prime}, w, \lambda}=v^{l\left(w^{\prime}\right)-l(w)} \sum_{i \in \mathbf{Z}} N_{i, w^{\prime}, w, \lambda} v^{i}$, that is,

$$
p_{z^{\prime}, z}^{\lambda}=v^{l\left(w^{\prime}\right)-l(w)} \sum_{i \in \mathbf{Z}} N_{i, w^{\prime}, w, \lambda} v^{i}
$$


if $w^{\prime} \mathbf{W}_{\lambda}=w \mathbf{W}_{\lambda}$ and $z, z^{\prime}$ are as in (d), then

$$
N_{i, w^{\prime}, w, \lambda}=0 \text { if } w^{\prime} \mathbf{W}_{\lambda} \neq w \mathbf{W}_{\lambda} .
$$

Note that $N_{i, w^{\prime}, w, \lambda}$ is 0 unless $i$ is even.

40.3. Let $B^{*} \in \mathcal{B}$. Let $U^{*}=U_{B^{*}}$ and let $T$ be a maximal torus of $B^{*}$. Let $\mathbf{r}=\operatorname{dim} \mathbf{T}$. Let $W_{T}=N_{G^{0}} T / T$. We identify $T=\mathbf{T}, W_{T}=\mathbf{W}$ as in 28.5. For any $w \in \mathbf{W}$ we denote by $\dot{w}$ a representative of $w$ in $N_{G^{0}} T$.

Let $C=G^{0} / U^{*} \times G^{0} / U^{*}$. We have a partition $C=\bigcup_{w \in \mathbf{W}} C_{w}$ where

$$
C_{w}=\left\{\left(h U^{*}, h^{\prime} U^{*}\right) \in C ; h^{-1} h^{\prime} \in B^{*} \dot{w} B^{*}\right\} .
$$

For $w \in \mathbf{W}$ let $d_{w}=\operatorname{dim} C_{w}$ and let

$$
\bar{C}_{w}=\left\{\left(h U^{*}, h^{\prime} U^{*}\right) \in C ; h^{-1} h^{\prime} \in \overline{B^{*} \dot{w} B^{*}}\right\}
$$

(closure in $G^{0}$ ). Now $\bar{C}_{w}$ is an irreducible variety and we have a partition $\bar{C}_{w}=$ $\bigcup_{w^{\prime} ; w^{\prime} \leq w} C_{w^{\prime}}$ with $C_{w}$ smooth, open dense in $\bar{C}_{w}$.

Define $\gamma_{\dot{w}}: B^{*} \dot{w} B^{*} \rightarrow T$ by $\gamma_{\dot{w}}(g)=t$ where $g \in U^{*} \dot{w} t U^{*}$ with $t \in T$. Define $\psi: C_{w} \rightarrow T$ by $\psi\left(h U^{*}, h^{\prime} U^{*}\right)=\gamma_{\dot{w}}\left(h^{-1} h^{\prime}\right)$.

For $\mathcal{L} \in \mathfrak{s}$ we set $\mathcal{L}_{w}=\psi^{*} \mathcal{L}$, a local system on $C_{w}$. (Using 28.1(c) we see that the isomorphism class of $\psi^{*} \mathcal{L}$ is independent of the choice of $\dot{w}$.) Let $\mathcal{L}_{w}^{\sharp}=$ $\operatorname{IC}\left(\bar{C}_{w}, \mathcal{L}_{w}\right) \in \mathcal{D}\left(\bar{C}_{w}\right)$.

40.4. For $w \in \mathbf{W}, \mathcal{L} \in \mathfrak{s}$ let $\underline{\mathcal{L}}_{w}=j_{w !} \mathcal{L}_{w}, \underline{\mathcal{L}}_{w}^{\sharp}=\bar{j}_{w !} \mathcal{L}_{w}^{\sharp}$ where $j_{w}: C_{w} \rightarrow C$, $\bar{j}_{w}: \bar{C}_{w} \rightarrow C$ are the inclusions. Let $\hat{C}$ be the full subcategory of $\mathcal{D}(C)$ whose objects are the simple perverse sheaves on $C$ which are equivariant for the $G^{0} \times T \times T$ action

(a) $\left(x, t, t^{\prime}\right):\left(h U^{*}, h^{\prime} U^{*}\right) \mapsto\left(x h t^{n} U^{*}, x h^{\prime} t^{\prime n} U^{*}\right)$

on $C$ (for some $n \in \mathbf{N}_{\mathbf{k}}^{*}$ ) or equivalently, are isomorphic to $\underline{\mathcal{L}}_{w}^{\sharp}\left[d_{w}\right]$ for some $\mathcal{L} \in \mathfrak{s}$ and some $w \in \mathbf{W}$. Let $\mathcal{D}^{c s}(C)$ be the subcategory of $\mathcal{D}(C)$ whose objects are those $K \in \mathcal{D}(C)$ such that for any $j$, any simple subquotient of ${ }^{p} H^{j} K$ is in $\hat{C}$.

If $w, \mathcal{L}$ are as above, then $\underline{\mathcal{L}}_{w} \in \mathcal{D}^{c s}(C)$. Indeed this constructible sheaf is equivariant for the action (a) (for some $n$ ), hence so is each ${ }^{p} H^{j}\left(\underline{\mathcal{L}}_{w}\right)$.

We have a diagram $C \times C \stackrel{r}{\leftarrow}\left(G^{0} / U^{*}\right)^{3} \stackrel{s}{\rightarrow} C$ where

$$
\begin{aligned}
& r\left(h_{1} U^{*}, h_{2} U^{*}, h_{3} U^{*}\right)=\left(\left(h_{1} U^{*}, h_{2} U^{*}\right),\left(h_{2} U^{*}, h_{3} U^{*}\right)\right), \\
& s\left(h_{1} U^{*}, h_{2} U^{*}, h_{3} U^{*}\right)=\left(h_{1} U^{*}, h_{3} U^{*}\right) .
\end{aligned}
$$

We define a bi-functor $\mathcal{D}(C) \times \mathcal{D}(C) \rightarrow \mathcal{D}(C)$ by $A, A^{\prime} \mapsto A * A^{\prime}=s ! r^{*}\left(A \otimes A^{\prime}\right)$. The "product" $A * A^{\prime}$ is associative in an obvious sense. We show that

(b) $A, A^{\prime} \mapsto A * A^{\prime}$ restricts to a bi-functor $\mathcal{D}^{c s}(C) \times \mathcal{D}^{c s}(C) \rightarrow \mathcal{D}^{c s}(C)$.

Let $A, A^{\prime} \in \mathcal{D}^{c s}(C)$. To show that $A * A^{\prime} \in \mathcal{D}^{c s}(C)$ we may assume that $A, A^{\prime} \in \hat{C}$. Then each ${ }^{p} H^{j}\left(A * A^{\prime}\right)$ is equivariant for the action (a) (for some $n$ ). This proves (b).

40.5. For $w^{\prime} \leq w$ in $\mathbf{W}, \lambda \in \underline{\mathfrak{s}}, \mathcal{L} \in \lambda$ and $i \in \mathbf{Z}$ we show that

(a) $\left.\mathcal{H}^{i}\left(\mathcal{L}_{w}^{\sharp}\right)\right|_{C_{w^{\prime}}} \cong\left(\mathcal{L}_{w^{\prime}}(-i / 2)\right)^{\oplus N_{i, w^{\prime}, w, \lambda}}$.

(Both sides are 0 unless $i$ is even.) 
Let

$$
\begin{aligned}
& \tilde{C}_{w}=\left\{\left(h, h^{\prime}\right) \in G^{0} \times G^{0} ; h^{-1} h^{\prime} \in B^{*} \dot{w} B^{*}\right\} \times \mathbf{k}^{*}, \\
& \tilde{\tilde{C}}_{w}=\left\{\left(h, h^{\prime}\right) \in G^{0} \times G^{0} ; h^{-1} h^{\prime} \in \overline{B^{*} \dot{w} B^{*}}\right\} \times \mathbf{k}^{*} .
\end{aligned}
$$

Now $\overline{\tilde{C}}_{w}$ is an irreducible variety and we have a partition $\overline{\tilde{C}}_{w}=\bigcup_{w^{\prime} ; w^{\prime} \leq w} \tilde{C}_{w^{\prime}}$ with $\tilde{C}_{w}$ smooth, open dense in $\overline{\tilde{C}}_{w}$. Define $\bar{d}: \overline{\tilde{C}}_{w} \rightarrow \bar{C}_{w}, d: \tilde{C}_{w} \rightarrow \bar{C}_{w}$ by $\left(h, h^{\prime}, z\right) \mapsto$ $\left(h U^{*}, h^{\prime} U^{*}\right)$. Let $\tilde{\mathcal{L}}_{w}=d^{*} \mathcal{L}_{w}$, a local system on $\tilde{C}_{w}$. Let $\tilde{\mathcal{L}}_{w}^{\sharp}=\operatorname{IC}\left(\tilde{\tilde{C}}_{w}, \tilde{\mathcal{L}}_{w}\right)$. Since $d, \bar{d}$ are principal $U^{*} \times \mathbf{k}^{*}$-bundles, it is enough to show that

(b) $\left.\mathcal{H}^{i}\left(\tilde{\mathcal{L}}_{w}^{\sharp}\right)\right|_{\tilde{C}_{w^{\prime}}} \cong\left(\tilde{\mathcal{L}}_{w^{\prime}}(-i / 2)\right)^{\oplus N_{i, w^{\prime}, w, \lambda}}$.

(Both sides are 0 unless $i$ is even.)

We choose $\kappa \in \operatorname{Hom}\left(T, \mathbf{k}^{*}\right), \mathcal{E} \in \mathfrak{s}\left(\mathbf{k}^{*}\right)$ such that $\mathcal{L} \cong \kappa^{*} \mathcal{E}$; see 28.1(c).

Now $B^{*}$ acts on $\left(B^{*} \dot{w} B^{*}\right) \times \mathbf{k}^{*}$ and on $\left(\overline{B^{*} \dot{w} B^{*}}\right) \times \mathbf{k}^{*}$ by

$$
t_{1} u:(g, z) \mapsto\left(g\left(t_{1} u\right)^{-1}, \kappa\left(t_{1}\right) z\right)
$$

where $t_{1} \in T, u \in U^{*}$. Let $\overline{\mathbf{P}}_{w}^{\kappa}=\left(\left(\overline{B^{*} \dot{w} B^{*}}\right) \times \mathbf{k}^{*}\right) / B^{*}, P P_{w}^{\kappa}=\left(\left(B^{*} \dot{w} B^{*}\right) \times \mathbf{k}^{*}\right) / B^{*}$. Now $\mathbf{P}_{w}^{\kappa}$ is a smooth open dense subvariety of the irreducible variety $\overline{\mathbf{P}}_{w}^{k}$ and $\overline{\mathbf{P}}_{w}^{\kappa}=\bigcup_{w^{\prime} ; w^{\prime} \leq w} \mathbf{P}_{w^{\prime}}^{\kappa}$ is a partition. The morphism $\left(B^{*} \dot{w} B^{*}\right) \times \mathbf{k}^{*} \rightarrow \mathbf{k}^{*}$ given by $(g, z) \mapsto \kappa\left(\gamma_{\dot{w}}(g)\right) z$ factors through a morphism $\phi: \mathbf{P}_{w}^{\kappa} \rightarrow \mathbf{k}^{*}$. Let $\mathcal{E}_{w}^{\kappa}=\phi^{*} \mathcal{E}$, a local system of rank 1 on $\mathbf{P}_{w}^{\kappa}$. Let $\mathcal{E}_{w}^{\kappa \sharp}=I C\left(\overline{\mathbf{P}}_{w}^{\kappa}, \mathcal{E}_{w}^{\kappa}\right) \in \mathcal{D}\left(\overline{\mathbf{P}}_{w}^{\kappa}\right)$. From [L14, 1.24] we see that

(c) $\left.\mathcal{H}^{i}\left(\mathcal{E}_{w}^{\kappa \sharp}\right)\right|_{\mathbf{P}_{w^{\prime}}^{\kappa}} \cong\left(\mathcal{E}_{w^{\prime}}^{\kappa}(-i / 2)\right)^{\oplus N_{i, w^{\prime}, w, \lambda}}$.

(Both sides are 0 unless $i$ is even.)

We can find $n \in \mathbf{N}_{\mathbf{k}}^{*}$ such that $\mathcal{E} \in \mathfrak{s}_{n}\left(\mathbf{k}^{*}\right)$. Define $\bar{c}: \overline{\tilde{C}}_{w} \rightarrow \overline{\mathbf{P}}_{w}, c: \overline{\tilde{C}}_{w} \rightarrow \overline{\mathbf{P}}_{w}$ by $\left(h, h^{\prime}, z\right) \mapsto B^{*}-$ orbit of $\left(h^{-1} h^{\prime}, z^{n}\right)$. Now $\bar{c}, c$ are locally trivial fibrations with smooth fibres of pure dimension. Hence (b) follows from (c) provided that we can show that $c^{*} \mathcal{E}_{w^{\prime}}^{\kappa}=\tilde{\mathcal{L}}_{w^{\prime}}$ for $w^{\prime} \leq w$. We may assume that $w=w^{\prime}$. We have a commutative diagram

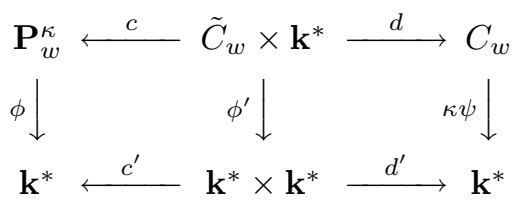

with $\phi, \psi, c, d$ as above, $\phi^{\prime}\left(h, h^{\prime}, z\right)=\left(\kappa\left(\gamma_{\dot{w}}\left(h^{-1} h^{\prime}\right)\right), z\right), c^{\prime}\left(z^{\prime}, z\right)=z^{\prime} z^{n}, d^{\prime}\left(z^{\prime}, z\right)=$ $z^{\prime}$. Using this and the definitions we have $\tilde{\mathcal{L}}_{w}=\phi^{\prime *} d^{\prime *} \mathcal{E}, c^{*} \mathcal{E}_{w}=\phi^{*} c^{\prime *} \mathcal{E}$. It remains to show that $d^{\prime *} \mathcal{E}=c^{\prime *} \mathcal{E}$. This expresses the fact that $\mathcal{E}$ is equivariant for the $\mathbf{k}^{*}$-action $z_{1}: z \mapsto z_{1}^{n} z$ on $\mathbf{k}^{*}$ which follows from $\mathcal{E} \in \mathfrak{s}_{n}\left(\mathbf{k}^{*}\right)$. This proves (b), hence (a).

40.6. Let $w, w^{\prime} \in \mathbf{W}, \mathcal{L}, \mathcal{L}^{\prime} \in \mathfrak{s}$. We set $L=\underline{\mathcal{L}}_{w} * \underline{\mathcal{L}}_{w^{\prime}}^{\prime} \in \mathcal{D}^{c s}(C)$. Let

$$
\begin{gathered}
X=\left\{\left(h_{1} U^{*}, h_{2} U^{*}, h_{3} U^{*}\right) \in\left(G^{0} / U^{*}\right)^{3} ; h_{1}^{-1} h_{2} \in B^{*} \dot{w} B^{*}, h_{2}^{-1} h_{3} \in B^{*} \dot{w}^{\prime} B^{*}\right\}, \\
\bar{X}=\left\{\left(h_{1} U^{*}, h_{2} B^{*}, h_{3} U^{*}\right) \in G^{0} / U^{*} \times G^{0} / B^{*} \times G^{0} / U^{*} ;\right. \\
\left.h_{1}^{-1} h_{2} \in B^{*} \dot{w} B^{*}, h_{2}^{-1} h_{3} \in B^{*} \dot{w}^{\prime} B^{*}\right\} .
\end{gathered}
$$


We have a commutative diagram with a cartesian square

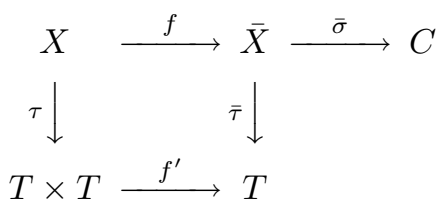

where $f$ is given by $\left(h_{1} U^{*}, h_{2} U^{*}, h_{3} U^{*}\right) \mapsto\left(h_{1} U^{*}, h_{2} B^{*}, h_{3} U^{*}\right)$,

$f^{\prime}$ is $\left(t, t^{\prime}\right) \mapsto \operatorname{Ad}\left(\dot{w}^{\prime}\right)^{-1}(t) t^{\prime}$,

$\tau$ is $\left(h_{1} U^{*}, h_{2} U^{*}, h_{3} U^{*}\right) \mapsto\left(t, t^{\prime}\right)$ with $h_{1}^{-1} h_{2} \in U^{*} \dot{w} t U^{*}, h_{2}^{-1} h_{3} \in U^{*} \dot{w}^{\prime} t^{\prime} U^{*}$,

$\bar{\tau}$ is $\left(h_{1} U^{*}, h_{2} B^{*}, h_{3} U^{*}\right) \mapsto \operatorname{Ad}\left(\dot{w}^{\prime}\right)^{-1}(t) t^{\prime}$ with $t, t^{\prime}$ as in the definition of $\tau$,

$\bar{\sigma}$ is $\left(h_{1} U^{*}, h_{2} B^{*}, h_{3} U^{*}\right) \mapsto\left(h_{1} U^{*}, h_{3} U^{*}\right)$.

From the definitions we have $L=\bar{\sigma}_{!} f_{!} \tau^{*}\left(\mathcal{L} \otimes \mathcal{L}^{\prime}\right)$. Using the diagram above, we have $L=\bar{\sigma}_{!} \bar{\tau}^{*} f_{!}^{\prime}\left(\mathcal{L} \otimes \mathcal{L}^{\prime}\right)$. From the definitions we see that either (i) or (ii) below holds:

(i) $\mathcal{L} \neq\left(\operatorname{Ad}\left(\dot{w}^{\prime}\right)^{-1}\right)^{*} \mathcal{L}^{\prime}$ and $f_{!}^{\prime}\left(\mathcal{L} \otimes \mathcal{L}^{\prime}\right)=0$;

(ii) $\mathcal{L} \cong\left(\operatorname{Ad}\left(\dot{w}^{\prime}\right)^{-1}\right)^{*} \mathcal{L}^{\prime}$ and $\mathcal{L} \otimes \mathcal{L}^{\prime}=f^{\prime *} \mathcal{L}^{\prime}$.

If (i) holds, then $K=0$. If (ii) holds, then, as in 32.16 , we have

$$
\begin{gathered}
f_{!}^{\prime}\left(\mathcal{L} \otimes \mathcal{L}^{\prime}\right)=f_{!}^{\prime} f^{\prime *} \mathcal{L}^{\prime}=\mathcal{L}^{\prime} \otimes f_{!}^{\prime} \overline{\mathbf{Q}}_{l} \approx\left\{\mathcal{L}^{\prime} \otimes \mathcal{H}^{e}\left(f_{!}^{\prime} \overline{\mathbf{Q}}_{l}\right)[-e], e \in \mathbf{Z}\right\}, \\
\mathcal{L}^{\prime} \otimes \mathcal{H}^{e}\left(f_{!}^{\prime} \overline{\mathbf{Q}}_{l}\right)[-e] \approx\left\{\mathcal{L}^{\prime}(\mathbf{r}-e), \ldots, \mathcal{L}^{\prime}(\mathbf{r}-e),\left(\left(\begin{array}{c}
\mathbf{r} \\
2 \mathbf{r}-e
\end{array}\right) \text { copies }\right)\right\} .
\end{gathered}
$$

Setting $\bar{L}=\bar{\sigma}_{!} \bar{\tau}^{*}\left(\mathcal{L}^{\prime}\right)$, it follows that

$$
L \approx\left\{\bar{L}(\mathbf{r}-e)[-e], \ldots, \bar{L}(\mathbf{r}-e)[-e],\left(\left(\begin{array}{c}
\mathbf{r} \\
2 \mathbf{r}-e
\end{array}\right) \text { copies }\right), e \in \mathbf{Z}\right\} .
$$

We now consider $\bar{L}$ for certain choices of $w, w^{\prime}$.

If $w, w^{\prime}$ satisfy $l\left(w w^{\prime}\right)=l(w)+l\left(w^{\prime}\right)$, then $\bar{\sigma}$ restricts to an isomorphism $\bar{X} \rightarrow$ $C_{w w^{\prime}}$ and $\bar{L}=\underline{\mathcal{L}}_{w w^{\prime}}^{\prime}$.

Now assume that $\alpha, \check{\alpha}, s_{\alpha}$ are as in 28.3 and that $w=w^{\prime}=s_{\alpha} \in \mathbf{I}$. We have

$$
\bar{L} \approx\left\{j_{u} \bar{L}_{u} ; u \in \mathbf{W}\right\}
$$

where $j_{u}: C_{u} \rightarrow C$ is the inclusion and $\bar{L}_{u}=j_{u}^{*} \bar{L}$. Let $\bar{X}_{u}=\bar{\sigma}^{-1}\left(C_{u}\right)$. Then $\bar{L}_{u}=\bar{\sigma}_{u} ! \bar{\tau}_{u}^{*}\left(\mathcal{L}^{\prime}\right)$ where $\bar{\sigma}_{u}: \bar{X}_{u} \rightarrow C_{u}, \bar{\tau}_{u}: \bar{X}_{u} \rightarrow T$ are the restrictions of $\bar{\sigma}, \bar{\tau}$.

If $u \notin\left\{1, s_{\alpha}\right\}$, then $\bar{X}_{u}=\emptyset$ and $\bar{L}_{u}=0$. If $u=1$, then $\bar{\sigma}_{u}: \bar{X}_{u} \rightarrow C_{u}$ is an affine line bundle and $\bar{\tau}_{u}^{*}\left(\mathcal{L}^{\prime}\right)=\bar{\sigma}_{u}^{*} \mathcal{L}_{u}^{\prime}$; hence $\bar{\sigma}_{u !} \bar{\tau}_{u}^{*}\left(\mathcal{L}^{\prime}\right)=\bar{\sigma}_{u !} \bar{\sigma}_{u}^{*} \mathcal{L}_{u}^{\prime}=\mathcal{L}_{u}^{\prime}[[-1]]$. If $u=s_{\alpha}$, then $\bar{\sigma}_{u}: \bar{X}_{u} \rightarrow C_{u}$ is a principal $\mathbf{k}^{*}$-bundle and either (iii) or (iv) below holds:

(iii) $\check{\alpha}^{*} \mathcal{L}^{\prime} \neq \overline{\mathbf{Q}}_{l}$ and $\bar{\sigma}_{u} \bar{\tau}_{u}^{*}\left(\mathcal{L}^{\prime}\right)=0$,

(iv) $\check{\alpha}^{*} \mathcal{L}^{\prime} \cong \overline{\mathbf{Q}}_{l}$ and $\bar{\tau}_{u}^{*}\left(\mathcal{L}^{\prime}\right)=\bar{\sigma}_{u}^{*} \mathcal{L}_{u}^{\prime}$.

If (iv) holds, then, as in case (ii) above, we have

$$
\begin{aligned}
& \bar{\sigma}_{u !} \bar{\tau}_{u}^{*}\left(\mathcal{L}^{\prime}\right)=\bar{\sigma}_{u !} \bar{\sigma}_{u}^{*} \mathcal{L}_{u}^{\prime}=\mathcal{L}_{u}^{\prime} \otimes \bar{\sigma}_{u !} \overline{\mathbf{Q}}_{l} \approx\left\{\mathcal{L}_{u}^{\prime} \otimes \mathcal{H}^{e}\left(\bar{\sigma}_{u !} \overline{\mathbf{Q}}_{l}\right)[-e], e \in \mathbf{Z}\right\} \\
& \mathcal{L}_{u}^{\prime} \otimes \mathcal{H}^{e}\left(\bar{\sigma}_{u !} \overline{\mathbf{Q}}_{l}\right)[-e] \approx\left\{\mathcal{L}_{u}^{\prime}(1-e), \ldots, \mathcal{L}_{u}^{\prime}(1-e),\left(\left(\begin{array}{c}
1 \\
2-e
\end{array}\right) \text { copies }\right)\right\}
\end{aligned}
$$


40.7. In this subsection we assume that $\mathbf{k}$ is an algebraic closure of a finite field. Now the $\mathcal{A}$-module $\mathfrak{K}(C)$ is defined as in 36.8 (the character sheaves on $C$ are taken to be the objects in $\hat{C}$ ).

For $(w, \lambda) \in \mathbf{W} \times \underline{\mathfrak{s}}$, let $[w ; \lambda]$ be the basis element of $\mathfrak{K}(C)$ given by $\underline{\mathcal{L}}_{w}^{\sharp}\left[\left[d_{w} / 2\right]\right]$; we choose $\mathcal{L} \in \lambda$ and we regard $\underline{\mathcal{L}}_{w}, \underline{\mathcal{L}}_{w}^{\sharp}$ as mixed complexes on $C$ whose restriction to $C_{w}$ is pure of weight 0 ; then $\operatorname{gr}\left(\underline{\mathcal{L}}_{w}\right), \operatorname{gr}\left(\underline{\mathcal{L}}_{w}^{\sharp}\right)$ are defined in $\mathfrak{K}(C)$ as in 36.8. We denote these elements of $\mathfrak{K}(C)$ by $[w ; \lambda]^{\prime},[w ; \lambda]^{\prime \sharp}$ respectively. From $40.5($ a) we see that

(a) $(-v)^{d_{w}}[w ; \lambda]=[w ; \lambda]^{\sharp \sharp}=\sum_{w^{\prime} \in \mathbf{W}} \sum_{i \in 2 \mathbf{Z}} N_{i, w^{\prime}, w, \lambda} v^{i}\left[w^{\prime} ; \lambda\right]^{\prime}$ in $\mathfrak{K}(C)$

where $N_{i, w^{\prime}, w, \lambda}$ is as in $40.2(\mathrm{e})$.

Let $r, s$ be as in 40.4. By 40.4(b), $s ! r^{*}: \mathcal{D}(C \times C) \rightarrow \mathcal{D}(C)$ restricts to a functor $\mathcal{D}^{c s}(C \times C) \rightarrow \mathcal{D}^{c s}(C)$ where the character sheaves on $C \times C$ are by definition complexes of the form $A \otimes A^{\prime}$ with $A \in \hat{C}, A^{\prime} \in \hat{C}$. Hence the $\mathcal{A}$-linear map $\operatorname{gr}\left(s ! r^{*}\right): \mathfrak{K}(C \times C) \rightarrow \mathfrak{K}(C)$ or equivalently $\mathfrak{K}(C) \otimes_{\mathcal{A}} \mathfrak{K}(C) \rightarrow \mathfrak{K}(C)$ is well defined. (We have canonically $\mathfrak{K}(C \times C)=\mathfrak{K}(C) \otimes_{\mathcal{A}} \mathfrak{K}(C)$.) We write $\xi * \xi^{\prime}$ instead of $\operatorname{gr}\left(s ! r^{*}\right)\left(\xi \otimes \xi^{\prime}\right)$ where $\xi, \xi^{\prime} \in \mathfrak{K}(C)$. Note that $\xi, \xi^{\prime} \mapsto \xi * \xi^{\prime}$ defines an associative $\mathcal{A}$-algebra structure on $\mathfrak{K}(C)$.

Let $w, w^{\prime} \in \mathbf{W}, \lambda, \lambda^{\prime} \in \underline{\mathfrak{s}}$. From 40.6 we see that

if $w^{\prime} \lambda^{\prime} \neq \lambda$, then $[w ; \lambda]^{\prime} *\left[w^{\prime} ; \lambda^{\prime}\right]^{\prime}=0$ in $\mathfrak{K}(C)$;

if $w^{\prime} \lambda^{\prime}=\lambda$ and $l\left(w w^{\prime}\right)=l(w)+l\left(w^{\prime}\right)$, then $[w ; \lambda]^{\prime} *\left[w^{\prime}, \lambda^{\prime}\right]^{\prime}=\left(v^{2}-1\right)^{\mathbf{r}}\left[w w^{\prime} ; \lambda^{\prime}\right]^{\prime}$ in $\mathfrak{K}(C)$;

if $s \in \mathbf{I}$ and $s \lambda^{\prime}=\lambda$, then $[s ; \lambda]^{\prime} *\left[s, \lambda^{\prime}\right]^{\prime}=\left(v^{2}-1\right)^{\mathbf{r}}\left(v^{2}\left[1 ; \lambda^{\prime}\right]^{\prime}+\left(v^{2}-1\right) c\left[s ; \lambda^{\prime}\right]^{\prime}\right)$ where $c=1$ for $s \in \mathbf{W}_{\lambda^{\prime}}$ and $c=0$ for $s \notin \mathbf{W}_{\lambda^{\prime}}$.

Using this and (a), 40.1(a), 40.2(e), we see that

(b) the unique $\mathcal{A}$-linear isomorphism $\omega: \mathfrak{K}(C) \rightarrow H$ ( $H$ as in 40.1) given by $[w, \lambda]^{\prime} \mapsto v^{l(w)} \tilde{T}_{w} 1_{\lambda}$ for $w \in \mathbf{W}, \lambda \in \underline{\mathfrak{s}}$, satisfies $\omega([w, \lambda])=(-v)^{-d_{w}} v^{l(w)} c_{w, \lambda}$ for $w \in \mathbf{W}, \lambda$ in $\underline{\mathfrak{s}}$ and $\omega\left(x * x^{\prime}\right)=\left(v^{2}-1\right)^{\mathbf{r}} \omega(x) \omega\left(x^{\prime}\right)$ for any $x, x^{\prime} \in \mathfrak{K}(C)$.

40.8. For $w, w^{\prime} \in \mathbf{W}$ and $\lambda, \lambda^{\prime} \in \underline{\mathfrak{s}}$ we have $c_{w, \lambda} c_{w^{\prime}, \lambda^{\prime}}=\sum_{y \in \mathbf{W}, \nu \in \underline{\mathfrak{s}}} \gamma_{y, \nu}^{w, \lambda ; w^{\prime}, \lambda^{\prime}} c_{y, \lambda}$ in the algebra $H$. Here $\gamma_{y, \nu}^{w, \lambda ; w^{\prime}, \lambda^{\prime}} \in \mathcal{A}$. We have:

(a) $\gamma_{y, \nu}^{w, \lambda ; w^{\prime}, \lambda^{\prime}} \in \mathbf{N}\left[v, v^{-1}\right]$.

By the arguments in $34.4-34.10$ (with $D=G^{0}$ ) this is reduced to the analogous (well-known) statement for the structure constants of the algebra $H_{\lambda}^{D}$ with its basis $\left(c_{w}^{\lambda}\right)$ (see 34.2).

40.9. For any $J \subset \mathbf{I}$ let $H_{J}$ be the $\mathcal{A}$-submodule of $H$ spanned by $\left\{c_{w, \lambda} ; w \in\right.$ $\left.\mathbf{W}_{J}, \lambda \in \underline{\mathfrak{s}}\right\}$ or equivalently by $\left\{\tilde{T}_{w} 1_{\lambda} ; w \in \mathbf{W}_{J}, \lambda \in \underline{\mathfrak{s}}\right\}$. From the definitions we see that $H_{J}$ is a subalgebra of $H$. For any $J \subset \mathbf{I}, J^{\prime} \subset \mathbf{I}$ we define a relation $\preceq_{J, J^{\prime}}$ on $\mathbf{W} \times \underline{\mathfrak{s}}$ as follows. We say that $(y, \nu) \preceq J_{, J^{\prime}}(w, \lambda)$ if there exist $w_{1} \in \mathbf{W}_{J}, w_{2} \in \mathbf{W}_{J^{\prime}}$, $\lambda_{1}, \lambda_{2} \in \underline{\mathfrak{s}}$ such that in the expansion (in the algebra $H$ )

$$
c_{w_{1}, \lambda_{1}} c_{w, \lambda} c_{w_{2}, \lambda_{2}}=\sum_{y^{\prime} \in \mathbf{W}, \nu^{\prime} \in \underline{\mathfrak{s}}} a_{y^{\prime}, \nu^{\prime}} c_{y^{\prime}, \nu^{\prime}}
$$

(with $a_{y^{\prime}, \nu^{\prime}} \in \mathcal{A}$ ) we have $a_{y, \nu} \neq 0$.

Using the associativity of the product in $H$, the fact that $H_{J}, H_{J^{\prime}}$ are subalgebras of $H$ and 40.8(a), we see that $\preceq_{J, J^{\prime}}$ is transitive. Using the formula $c_{1, w \lambda} c_{w, \lambda} c_{1, \lambda}=c_{w, \lambda}$ we see that it is reflexive. Thus, it is a preorder. Let $\sim_{J, J^{\prime}}$ be the equivalence relation attached to $\preceq_{J, J^{\prime}}$; thus, $(y, \nu) \sim_{J, J^{\prime}}(w, \lambda)$ if 
$(y, \nu) \preceq_{J, J^{\prime}}(w, \lambda)$ and $(w, \lambda) \preceq_{J, J^{\prime}}(y, \nu)$. The equivalence classes for $\sim_{J, J^{\prime}}$ are called $\left(J, J^{\prime}\right)$-two-sided cells. The $(\mathbf{I}, \mathbf{I})$-two-sided cells in $\mathbf{W} \times \underline{\mathfrak{s}}$ are also called two-sided cells.

40.10. Let $w, w^{\prime}, w^{\prime \prime} \in \mathbf{W}, \mathcal{L}, \mathcal{L}^{\prime}, \mathcal{L}^{\prime \prime} \in \mathfrak{s}$. We set $K=\underline{\mathcal{L}}_{w} * \underline{\mathcal{L}}_{w^{\prime}}^{\prime} * \underline{\mathcal{L}}_{w^{\prime \prime}}^{\prime \prime} \in \mathcal{D}^{c s}(C)$. Let

$$
\begin{aligned}
& X=\left\{\left(h_{1} U^{*}, h_{2} U^{*}, h_{3} U^{*}, h_{4} U^{*}\right) \in\left(G^{0} / U^{*}\right)^{4} ;\right. \\
& \left.h_{1}^{-1} h_{2} \in B^{*} \dot{w} B^{*}, h_{2}^{-1} h_{3} \in \overline{B^{*} \dot{w}^{\prime} B^{*}}, h_{3}^{-1} h_{4} \in B^{*} \dot{w}^{\prime \prime} B^{*}\right\},
\end{aligned}
$$

an irreducible variety. Let $X_{0}$ be the smooth open dense subset of $X$ defined by the condition $h_{2}^{-1} h_{3} \in B^{*} \dot{w}^{\prime} B^{*}$. Define $\sigma: X \rightarrow C$ by

$$
\left(h_{1} U^{*}, h_{2} U^{*}, h_{3} U^{*}, h_{4} U^{*}\right) \mapsto\left(h_{1} U^{*}, h_{4} U^{*}\right) .
$$

Define $\tau: X_{0} \rightarrow T \times T \times T$ by

$$
\left(h_{1} U^{*}, h_{2} U^{*}, h_{3} U^{*}, h_{4} U^{*}\right) \mapsto\left(t, t^{\prime}, t^{\prime \prime}\right)
$$

with $h_{1}^{-1} h_{2} \in U^{*} \dot{w} t U^{*}, h_{2}^{-1} h_{3} \in U^{*} \dot{w}^{\prime} t^{\prime} U^{*}, h_{3}^{-1} h_{4} \in U^{*} \dot{w}^{\prime \prime} t^{\prime \prime} U^{*}$.

Let $\mathcal{F}=\tau^{*}\left(\mathcal{L} \otimes \mathcal{L}^{\prime} \otimes \mathcal{L}^{\prime \prime}\right)$, a local system on $X_{0}$. Then $\mathcal{F}^{\sharp}:=I C(X, \mathcal{F}) \in \mathcal{D}(X)$ is defined and we have $K=\sigma ! \mathcal{F}^{\sharp}$.

Let $\bar{X}\left(\right.$ resp. $\left.\bar{X}_{0}\right)$ be the the variety of all $\left(h_{1} U^{*}, h_{2} B^{*}, h_{3} B^{*}, h_{4} U^{*}\right) \in G^{0} / U^{*} \times$ $G^{0} / B^{*} \times G^{0} / B^{*} \times G^{0} / U^{*}$ that satisfy the same equations as those defining $X$ (resp. $\left.X_{0}\right)$. Note that $\bar{X}$ is irreducible and $\bar{X}_{0}$ is an open dense smooth subset of $\bar{X}$. We have a cartesian diagram

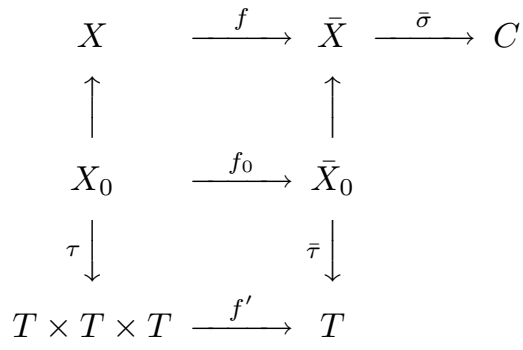

where $X_{0} \rightarrow X, \bar{X}_{0} \rightarrow \bar{X}$ are the obvious imbeddings,

$f, f_{0}$ are given by $\left(h_{1} U^{*}, h_{2} U^{*}, h_{3} U^{*}, h_{4} U^{*}\right) \mapsto\left(h_{1} U^{*}, h_{2} B^{*}, h_{3} B^{*}, h_{4} U^{*}\right)$,

$f^{\prime}$ is $\left(t, t^{\prime}, t^{\prime \prime}\right) \mapsto \operatorname{Ad}\left(\dot{w}^{\prime} \dot{w}^{\prime \prime}\right)^{-1}(t) \operatorname{Ad}\left(\dot{w}^{\prime \prime}\right)^{-1}\left(t^{\prime}\right) t^{\prime \prime}$,

$\bar{\tau}$ is $\left(h_{1} U^{*}, h_{2} B^{*}, h_{3} B^{*}, h_{4} U^{*}\right) \mapsto \operatorname{Ad}\left(\dot{w}^{\prime} \dot{w}^{\prime \prime}\right)^{-1}(t) \operatorname{Ad}\left(\dot{w}^{\prime \prime}\right)^{-1}\left(t^{\prime}\right) t^{\prime \prime}$ with $t, t^{\prime}, t^{\prime \prime}$ as in the definition of $\tau, \bar{\sigma}$ is $\left(h_{1} U^{*}, h_{2} B^{*}, h_{3} B^{*}, h_{4} U^{*}\right) \mapsto\left(h_{1} U^{*}, h_{4} U^{*}\right)$. Assume that $\mathcal{L} \cong\left(\operatorname{Ad}\left(\dot{w}^{\prime}\right)^{-1}\right)^{*} \mathcal{L}^{\prime}$ and $\mathcal{L}^{\prime} \cong\left(\operatorname{Ad}\left(\dot{w}^{\prime \prime}\right)^{-1}\right)^{*} \mathcal{L}^{\prime \prime}$. Then $\mathcal{L} \otimes \mathcal{L}^{\prime} \otimes \mathcal{L}^{\prime \prime}=f^{\prime *} \mathcal{L}^{\prime \prime}$. We have $\mathcal{F}=\tau^{*} f^{\prime *} \mathcal{L}^{\prime \prime}=f_{0}^{*} \bar{\tau}^{*} \mathcal{L}^{\prime \prime}$. Since $f$ is a principal $T \times T$-bundle and $X_{0}=f^{-1}\left(\bar{X}_{0}\right)$ it follows that $\mathcal{F}^{\sharp}=f^{*} I C\left(\bar{X}, \bar{\tau}^{*} \mathcal{L}^{\prime \prime}\right)$. Note that $f_{!} \overline{\mathbf{Q}}_{l} \approx\left\{\mathcal{H}^{e}\left(f_{!} \overline{\mathbf{Q}}_{l}\right)[-e], 2 \mathbf{r} \leq e \leq 4 \mathbf{r}\right\}$,

$$
\mathcal{H}^{e}\left(f_{!} \overline{\mathbf{Q}}_{l}\right) \approx\left\{\overline{\mathbf{Q}}_{l}(2 \mathbf{r}-e), \ldots, \overline{\mathbf{Q}}_{l}(2 \mathbf{r}-e),\left(\left(\begin{array}{c}
2 \mathbf{r} \\
4 \mathbf{r}-e
\end{array}\right) \text { copies }\right)\right\} .
$$

Hence setting $\bar{K}=\bar{\sigma}_{!}\left(I C\left(\bar{X}, \bar{\tau}^{*} \mathcal{L}^{\prime \prime}\right)\right)$ we have

(a) $K \approx\left\{\bar{K}(2 \mathbf{r}-e)[-e], \ldots, \bar{K}(2 \mathbf{r}-e)[-e],\left(\left(\begin{array}{c}2 \mathbf{r} \\ 4 \mathbf{r}-e\end{array}\right)\right.\right.$ copies $\left.), 2 \mathbf{r} \leq e \leq 4 \mathbf{r}\right\}$.

We now show that

(b) if $A \in \hat{C}$ is such that $A \dashv \bar{K}$, then $A \dashv K$. 
We may regard $\mathcal{L}, \mathcal{L}^{\prime}, \mathcal{L}^{\prime \prime}$ as mixed local systems (with respect to a rational structure over a sufficiently large finite subfield of $\mathbf{k}$ ) which are pure of weight 0 . Then $K, \bar{K}$ are naturally mixed complexes and (a) is compatible with the mixed structures. For any mixed perverse sheaf $P$, let $P_{h}$ be the subquotient of $P$ of pure weight $h$. We can find $h \in \mathbf{Z}$ such that $A \dashv{ }^{p} H^{j}(\bar{K})_{h}$ for some $j \in \mathbf{Z}$; moreover, we may assume that $h$ is maximum possible. Note that $A \dashv{ }^{p} H^{j+4 \mathbf{r}}(\bar{K}[-4 \mathbf{r}](-2 \mathbf{r}))_{h+2 \mathbf{r}}$ and $A \rtimes^{p} H^{j^{\prime}}(\bar{K}[-e](2 \mathbf{r}-e))_{h+2 \mathbf{r}}$ for $2 \mathbf{r} \leq e<4 \mathbf{r}$ and any $j^{\prime}$; hence from (a) we see that $A \dashv{ }^{p} H^{j+4 \mathbf{r}}(K)_{h+2 \mathbf{r}}$. In particular, $A \dashv K$, and (b) is proved.

40.11. Let $w, w^{\prime} \mathcal{L}, \mathcal{L}^{\prime}, X, \bar{X}, \tau$ be as in 40.6 . We set $\mathbf{L}=\underline{\mathcal{L}}_{w}^{\sharp} * \underline{\mathcal{L}}_{w^{\prime}}^{\prime} \in \mathcal{D}^{c s}(C)$. Let $A=\underline{\mathcal{L}}_{w^{\prime \prime}}^{\prime \prime} \sharp\left[d_{w^{\prime \prime}}\right]$. We show that

(a) if $A \dashv \mathbf{L}$, then $\left[w^{\prime \prime}, \lambda^{\prime \prime}\right]$ appears with nonzero coefficient in the expansion of the product $[w, \lambda] *\left[w^{\prime}, \lambda^{\prime}\right]$ in terms of the basis $([y, \nu])$ of $\mathfrak{K}(C)$.

Let

$$
\begin{aligned}
\mathbf{X}= & \left\{\left(h_{1} U^{*}, h_{2} U^{*}, h_{3} U^{*}\right) \in\left(G^{0} / U^{*}\right)^{3} ; h_{1}^{-1} h_{2} \in \overline{B^{*} \dot{w} B^{*}}, h_{2}^{-1} h_{3} \in \overline{B^{*} \dot{w}^{\prime} B^{*}}\right\}, \\
\overline{\mathbf{X}}=\left\{\left(h_{1} U^{*}, h_{2} B^{*}, h_{3} U^{*}\right) \in G^{0} / U^{*} \times G^{0} / B^{*} \times G^{0} / U^{*} ;\right. & \\
& \left.h_{1}^{-1} h_{2} \in \overline{B^{*} \dot{w} B^{*}}, h_{2}^{-1} h_{3} \in \overline{B^{*} \dot{w}^{\prime} B^{*}}\right\} .
\end{aligned}
$$

Note that $X$ (resp. $\bar{X}$ ) is naturally an open dense subset of $\mathbf{X}$ (resp. $\overline{\mathbf{X}}$ ). Define $\sigma^{\prime}: \mathbf{X} \rightarrow C$ by $\left(h_{1} U^{*}, h_{2} U^{*}, h_{3} U^{*}\right) \mapsto\left(h_{1} U^{*}, h_{3} U^{*}\right)$. Define $\bar{\sigma}^{\prime}: \overline{\mathbf{X}} \rightarrow C$ by $\left(h_{1} U^{*}, h_{2} B^{*}, h_{3} U^{*}\right) \mapsto\left(h_{1} U^{*}, h_{3} U^{*}\right)$. Let $\mathcal{F}=\tau^{*}\left(\mathcal{L} \otimes \mathcal{L}^{\prime}\right)$, a local system on $X$. Then $\mathcal{F}^{\sharp}:=\operatorname{IC}(\mathbf{X}, \mathcal{F}) \in \mathcal{D}(\mathbf{X})$ is defined and we have $\mathbf{L}=\sigma_{!}^{\prime} \mathcal{F}^{\sharp}$. We have a cartesian diagram

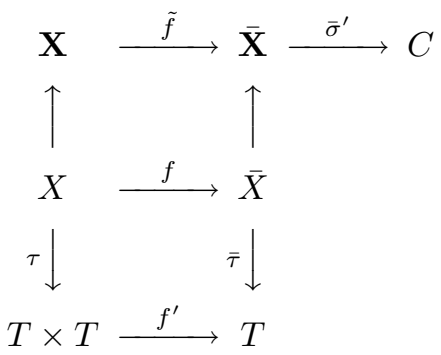

where $X \rightarrow \mathbf{X}, \bar{X} \rightarrow \overline{\mathbf{X}}$ are the obvious imbeddings, $f, f^{\prime}, \bar{\tau}$ are as in 40.6 and $\tilde{f}$ is the obvious map.

Assume first that 40.6(i) holds. Let $m^{\prime}: T \times \mathbf{X} \rightarrow \mathbf{X}$ be the free $T$-action $t_{1}:\left(h_{1} U^{*}, h_{2} U^{*}, h_{3} U^{*}\right) \mapsto\left(h_{1} U^{*}, h_{2} t_{1}^{-1} U^{*}, h_{3} U^{*}\right)$. This restricts to a free $T$-action $m: T \times X \rightarrow X$. Define a free $T$ action $m_{0}: T \times(T \times T) \rightarrow T \times T$ by $t_{1}:\left(t, t^{\prime}\right) \mapsto$ $\left(t_{1}^{-1} t, \operatorname{Ad}\left(\dot{w}^{\prime}\right)^{-1}\left(t_{1}\right) t^{\prime}\right.$. Then $m, m_{0}$ are compatible with $\tau$. By our assumption we have $m_{0}^{*}\left(\mathcal{L} \otimes \mathcal{L}^{\prime}\right)=\mathcal{L}_{0} \otimes \mathcal{L} \otimes \mathcal{L}^{\prime}$ where $\mathcal{L}_{0} \in \mathfrak{s}(T), \mathcal{L}_{0} \neq \overline{\mathbf{Q}}_{l}$. It follows that $m^{*}(\mathcal{F}) \cong \mathcal{L}_{0} \otimes \mathcal{F}$. From the properties of intersection cohomology we then have $m^{\prime *}\left(\mathcal{F}^{\sharp}\right) \cong \mathcal{L}_{0} \otimes \mathcal{F}^{\sharp}$. Let $r: T \times \mathbf{X} \rightarrow \mathbf{X}$ be the second projection. Since $\mathcal{L}_{0} \in \mathfrak{s}(T)$, $\mathcal{L}_{0} \nsucceq \overline{\mathbf{Q}}_{l}$, we have $r !\left(\mathcal{L}_{0} \otimes \mathcal{F}^{\sharp}\right)=0$. Hence $r ! m^{\prime *}\left(\mathcal{F}^{\sharp}\right)=0$. Since $m^{\prime}, f^{\prime}, r, f^{\prime}$ form a cartesian diagram we must have $f^{\prime *} f^{\prime}\left(\mathcal{F}^{\sharp}\right)=0$. Since $f^{\prime}$ is a principal $T$-bundle we deduce that $f_{!}^{\prime}\left(\mathcal{F}^{\sharp}\right)=0$. We have $\mathbf{L}=\bar{\sigma}_{!}^{\prime} f_{!}^{\prime}\left(\mathcal{F}^{\sharp}\right)$ hence $\mathbf{L}=0$. In this case (a) is clear.

Assume next that 40.6(ii) holds. Then $\mathcal{L} \otimes \mathcal{L}^{\prime}=f^{\prime *} \mathcal{L}^{\prime}$ and $\mathcal{F}=\tau^{*} f^{\prime *} \mathcal{L}^{\prime}=$ $f^{*} \bar{\tau}^{*} \mathcal{L}^{\prime}$. Since $f^{\prime}$ is a principal $T$-bundle and $X=f^{\prime-1}(\bar{X})$ it follows that $\mathcal{F}^{\sharp}=$ 
$f^{\prime *} I C\left(\overline{\mathbf{X}}, \bar{\tau}^{*} \mathcal{L}^{\prime}\right)$. Note that $f_{!}^{\prime} \overline{\mathbf{Q}}_{l} \approx\left\{\mathcal{H}^{e}\left(f_{!}^{\prime} \overline{\mathbf{Q}}_{l}\right)[-e], \mathbf{r} \leq e \leq 2 \mathbf{r}\right\}$

$$
\mathcal{H}^{e}\left(f_{!}^{\prime} \overline{\mathbf{Q}}_{l}\right) \approx\left\{\overline{\mathbf{Q}}_{l}(\mathbf{r}-e), \ldots, \overline{\mathbf{Q}}_{l}(\mathbf{r}-e),\left(\left(\begin{array}{c}
\mathbf{r} \\
2 \mathbf{r}-e
\end{array}\right) \text { copies }\right)\right\} .
$$

Hence setting $\overline{\mathbf{L}}=\bar{\sigma}_{!}^{\prime}\left(I C\left(\overline{\mathbf{X}}, \bar{\tau}^{*} \mathcal{L}^{\prime}\right)\right)$ we have

$$
\begin{aligned}
\mathbf{L} & =\sigma_{!}^{\prime} f^{\prime *} I C\left(\overline{\mathbf{X}}, \bar{\tau}^{*} \mathcal{L}^{\prime}\right)=\bar{\sigma}_{!}^{\prime} f_{!}^{\prime} f^{\prime *} I C\left(\overline{\mathbf{X}}, \bar{\tau}^{*} \mathcal{L}^{\prime}\right)=\bar{\sigma}_{!}^{\prime}\left(I C\left(\overline{\mathbf{X}}, \bar{\tau}^{*} \mathcal{L}^{\prime}\right) \otimes f_{!}^{\prime} \overline{\mathbf{Q}}_{l}\right), \\
\mathbf{L} & \approx\left\{\overline{\mathbf{L}}(\mathbf{r}-e)[-e], \ldots, \overline{\mathbf{L}}(\mathbf{r}-e)[-e],\left(\left(\begin{array}{c}
\mathbf{r} \\
2 \mathbf{r}-e
\end{array}\right) \text { copies }\right), \mathbf{r} \leq e \leq 2 \mathbf{r}\right\} .
\end{aligned}
$$

Since $A \dashv \mathbf{L}$, this shows that $A \dashv \overline{\mathbf{L}}$. We regard $\mathcal{L}^{\prime}$ as a pure local system of weight 0 . Then $\overline{\mathbf{L}}=\bar{\sigma}_{!}^{\prime}\left(I C\left(\overline{\mathbf{X}}, \bar{\tau}^{*} \mathcal{L}^{\prime}\right)\right)$ is again pure of weight 0 , since $\bar{\sigma}^{\prime}$ is proper (see (BBD). Hence the coefficient with which $A$ appears in the expansion of $\operatorname{gr}(\overline{\mathbf{L}})$ is a polynomial in $-v$ with coefficients given by the multiplicities of $A$ in the various ${ }^{p} H^{j}(\overline{\mathbf{L}})$; in particular, $A$ appears with coefficient $\neq 0$ in $\operatorname{gr}(\overline{\mathbf{L}})$. On the other hand, the arguments above show that $[w, \lambda] *\left[w^{\prime}, \lambda^{\prime}\right]=\left(v^{2}-1\right)^{\mathbf{r}} g r(\overline{\mathbf{L}})$. It follows that $A$ appears with coefficient $\neq 0$ in $[w, \lambda] *\left[w^{\prime}, \lambda^{\prime}\right]$. This proves (a).

\section{Character sheaves And TwO-Sided Cells}

41.1. In this section we preserve the notation of 40.3. We fix a connected component $D$ of $G$ and we pick $\delta \in N_{D} B^{*} \cap N_{D} T$. We write $\epsilon$ instead of $\epsilon_{D}: \mathbf{W} \rightarrow \mathbf{W}$. For $w \in \mathbf{W}$ we set

$$
Z_{\emptyset, D}^{w}=\left\{\left(B, B^{\prime}, x U_{B}\right) \in Z_{\emptyset, D} ; \operatorname{pos}\left(B, B^{\prime}\right)=w\right\} .
$$

(This is the same as ${ }^{w^{-1}} Z_{\emptyset, D}$ in 36.2.) Define $\xi_{D}: C \rightarrow Z_{\emptyset, D}$ by $\left(h U^{*}, h^{\prime} U^{*}\right) \mapsto$ $\left(h B^{*} h^{-1}, h^{\prime} B^{*} h^{-1}, h^{\prime} \delta h^{-1} U_{h B^{*} h^{-1}}\right)$, a principal $T$-bundle for the free $T$-action on $C$ given by $t:\left(h U^{*}, h^{\prime} U^{*}\right) \rightarrow\left(h t U^{*}, h^{\prime}\left(\delta t \delta^{-1}\right) U^{*}\right)$.

Since $\xi_{D}^{-1}\left(Z_{\emptyset, D}^{w}\right)=C_{w}, \xi_{D}$ restricts to a principal $T$-bundle $\xi_{D, w}: C_{w} \rightarrow Z_{\emptyset, D}^{w}$. We have a commutative diagram

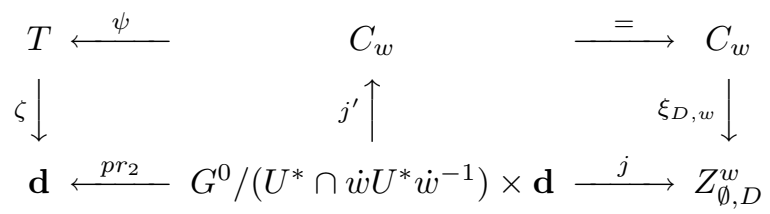

where $\psi$ is as in 40.3 ,

$$
\begin{aligned}
& \mathbf{d}=\dot{w} \delta T \\
& j\left(f\left(U^{*} \cap \dot{w} U^{*} \dot{w}^{-1}\right), s\right)=\left(f B^{*} f^{-1}, f \dot{w} B^{*} \dot{w}^{-1} f^{-1}, f s f^{-1} U_{f B^{*}-1}\right), \\
& j^{\prime}\left(f\left(U^{*} \cap \dot{w} U^{*} \dot{w}^{-1}\right), s\right)=\left(f U^{*}, f s \delta^{-1} U^{*}\right), \\
& \zeta(t)=\dot{w} \delta\left(\delta^{-1} t \delta\right) .
\end{aligned}
$$

Note that the lower row in the diagram is as in $36.2(\mathrm{a})$.

Define $\iota: \mathbf{d} \rightarrow T$ by $\iota(\dot{w} \delta t)=t$ where $t \in T$. If $\mathcal{L} \in \mathfrak{s}$ is such that $\operatorname{Ad}\left((\dot{w} d)^{-1}\right)^{*} \mathcal{L}$ $\cong \mathcal{L}$, then $\operatorname{pr}_{2}^{*} \iota^{*}(\mathcal{L})$ is a local system on $G^{0} /\left(U^{*} \cap \dot{w} U^{*} \dot{w}^{-1}\right) \times \mathbf{d}$, equivariant for the $T$-action $t_{0}:\left(f\left(U^{*} \cap \dot{w} U^{*} \dot{w}^{-1}\right), s\right)=\left(f t_{0}^{-1}\left(U^{*} \cap \dot{w} U^{*} \dot{w}^{-1}\right), t_{0} s t_{0}^{-1}\right)$ on $G^{0} /\left(U^{*} \cap\right.$ $\left.\dot{w} U^{*} \dot{w}^{-1}\right) \times \mathbf{d}$, which makes $j$ a principal $T$-bundle. It follows that there is a welldefined local system $\dot{\mathcal{L}}_{w}$ (of rank 1 ) on $Z_{\emptyset, D}^{w}$ such that $j^{*} \dot{\mathcal{L}}_{w}=p r_{2}^{*} \iota^{*}(\mathcal{L})$. We show that

(a) $\xi_{D, w}^{*}\left(\dot{\mathcal{L}}_{w}\right)=\left(\operatorname{Ad}\left(\delta^{-1}\right)^{*} \mathcal{L}\right)_{w}$. 
Since $j^{\prime}$ is an isomorphism, it is enough to show that $j^{\prime *} \xi_{D, w}^{*}\left(\dot{\mathcal{L}}_{w}\right)=j^{\prime *}\left(\operatorname{Ad}\left(\delta^{-1}\right)^{*} \mathcal{L}\right)_{w}$ or that $j^{*} \dot{\mathcal{L}}_{w}=j^{\prime *}\left(\operatorname{Ad}\left(\delta^{-1}\right)^{*} \mathcal{L}\right)_{w}$ or that $\left.p r_{2}^{*} \iota^{*} \mathcal{L}=j^{\prime *} \psi^{*}\left(\operatorname{Ad}\left(\delta^{-1}\right)^{*}\right) \mathcal{L}\right)$ or that $\left.j^{*} \psi^{*} \zeta^{*} \iota^{*} \mathcal{L}=j^{\prime *} \psi^{*}\left(\operatorname{Ad}\left(\delta^{-1}\right)^{*}\right) \mathcal{L}\right)$. It is enough to show that $\zeta^{*} \iota^{*} \mathcal{L}=\operatorname{Ad}\left(\delta^{-1}\right)^{*} \mathcal{L}$. This follows from $\operatorname{Ad}\left(\delta^{-1}\right)=\iota \zeta: T \rightarrow T$.

Let $h_{w}: Z_{\emptyset, D}^{w} \rightarrow Z_{\emptyset, D}, \bar{h}_{w}: \bar{Z}_{\emptyset, D}^{w} \rightarrow Z_{\emptyset, D}$ be the inclusions $\left(\bar{Z}_{\emptyset, D}^{w}=\bigcup_{w^{\prime} ; w^{\prime} \leq w} Z_{\emptyset, D}^{w^{\prime}}\right.$ is the closure of $Z_{\emptyset, D}^{w}$ in $\left.Z_{\emptyset, D}\right)$. Let $\underline{\mathcal{L}}_{w}=h_{w !} \dot{\mathcal{L}}_{w}, \underline{\mathcal{L}}_{w}^{\sharp}=\bar{h}_{w !} \dot{\mathcal{L}}_{w}^{\sharp}$. Using (a) and the fact that $\xi_{D}$ is a principal $T$-bundle we deduce

(b) $\xi_{D}^{*}\left(\underline{\dot{\mathcal{L}}}_{w}\right)=\underline{\left(\operatorname{Ad}\left(\delta^{-1}\right)^{*} \mathcal{L}\right)} w$,

(c) $\xi_{D}^{*}\left(\underline{\dot{\mathcal{L}}}_{w}^{\sharp}\right)=\underline{\left(\operatorname{Ad}\left(\delta^{-1}\right)^{*} \mathcal{L}\right)^{\sharp}} w$.

Now let $D^{\prime}$ be another connected component of $G$. We pick $\delta^{\prime} \in N_{D^{\prime}} B^{*} \cap N_{D^{\prime}} T$. We have a commutative diagram with a cartesian right square

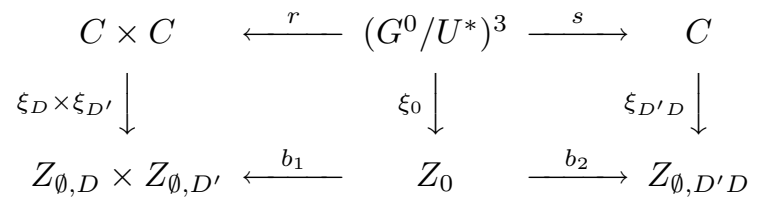

where $r, s$ are as in $40.4, Z_{0}, b_{1}, b_{2}$ are as in 32.5 (with $J=\emptyset$ ) and

$$
\begin{aligned}
& \xi_{0}\left(h_{1} U^{*}, h_{2} U^{*}, h_{3} U^{*}\right) \\
& =\left(h_{1} B^{*} h_{1}^{-1}, h_{2} B^{*} h_{2}^{-1}, h_{3} B^{*} h_{3}^{-1}, h_{2} \delta h_{1}^{-1} U_{h_{1} B^{*} h_{1}^{-1}}, h_{3} \delta^{\prime} h_{2}^{-1} U_{h_{2} B^{*} h_{2}^{-1}}\right) .
\end{aligned}
$$

Hence, if $A \in \mathcal{D}\left(Z_{\emptyset, D}\right), A^{\prime} \in \mathcal{D}\left(Z_{\emptyset, D^{\prime}}\right)$, then $\xi_{D^{\prime} D}^{*} b_{2 !} b_{1}^{*}\left(A \otimes A^{\prime}\right)=s_{!} r^{*}\left(\xi_{D}^{*} A \otimes \xi_{D^{\prime}}^{*} A^{\prime}\right)$, or equivalently

(d) $\xi_{D^{\prime} D}^{*}\left(A * A^{\prime}\right)=\left(\xi_{D}^{*} A\right) *\left(\xi_{D^{\prime}}^{*} A^{\prime}\right)$.

41.2. Let $u \in \mathbf{W}$. Let

$$
\begin{aligned}
\Upsilon_{u}= & \left\{\left(B, B^{\prime}, g\left(U_{B} \cap U_{B^{\prime}}\right) ;\right.\right. \\
& \left.B \in \mathcal{B}, B^{\prime} \in \mathcal{B}, g\left(U_{B} \cap U_{B^{\prime}}\right) \in D /\left(U_{B} \cap U_{B^{\prime}}\right), \operatorname{pos}\left(B, B^{\prime}\right)=u\right\}
\end{aligned}
$$

and let $\Phi_{u}: \mathcal{D}\left(Z_{\emptyset, D}\right) \rightarrow \mathcal{D}\left(Z_{\emptyset, D}\right)$ be the composition $\mathfrak{h}_{!} \mathfrak{j}^{*}$ where $\mathfrak{j}: \Upsilon_{u} \rightarrow Z_{\emptyset, D}$ is $\left(B, B^{\prime}, g\left(U_{B} \cap U_{B^{\prime}}\right) \mapsto\left(B, g B g^{-1}, g U_{B}\right)\right.$ and $\mathfrak{h}: \Upsilon_{u} \rightarrow Z_{\emptyset, D}$ is

$$
\left(B, B^{\prime}, g\left(U_{B} \cap U_{B^{\prime}}\right) \mapsto\left(B^{\prime}, g B^{\prime} g^{-1}, g U_{B^{\prime}}\right) .\right.
$$

(A special case of definitions in 37.1.) Let

$$
\begin{gathered}
\Upsilon^{\prime}=\left\{\left(B^{\prime}, B, \tilde{B}, \tilde{B}^{\prime}, g U_{B^{\prime}}\right) ; B^{\prime} \in \mathcal{B}, B \in \mathcal{B}, \tilde{B} \in \mathcal{B}, \tilde{B}^{\prime} \in \mathcal{B},\right. \\
\left.g U_{B^{\prime}} \in D / U_{B^{\prime}}, \operatorname{pos}\left(B^{\prime}, B\right)=u^{-1}, \operatorname{pos}\left(\tilde{B}, \tilde{B}^{\prime}\right)=\epsilon(u), g B^{\prime} g^{-1}=\tilde{B}^{\prime}\right\}, \\
s: \Upsilon_{u} \rightarrow \Upsilon^{\prime},\left(B, B^{\prime}, g\left(U_{B} \cap U_{B^{\prime}}\right) \mapsto\left(B^{\prime}, B, g B g^{-1}, g B^{\prime} g^{-1}, g U_{B^{\prime}}\right) .\right.
\end{gathered}
$$

Note that $s$ is an isomorphism. (We show this only at the level of sets. Define $s^{\prime}: \Upsilon^{\prime} \rightarrow \Upsilon_{u}$ by $\left(B^{\prime}, B, \tilde{B}, \tilde{B}^{\prime}, g U_{B^{\prime}}\right) \mapsto\left(B, B^{\prime}, x\left(U_{B} \cap U_{B^{\prime}}\right)\right)$ where $x \in D$ is such that $x B x^{-1}=\tilde{B}, x U_{B^{\prime}}=g U_{B^{\prime}}$. This is well defined and clearly an inverse of $s$.) It follows that $\mathfrak{h}_{:} j^{*}=\mathfrak{h}_{!}^{\prime} \mathfrak{j}^{\prime *}$ where

$$
\begin{aligned}
& \mathfrak{h}^{\prime}=\mathfrak{h} s^{\prime}: \Upsilon^{\prime} \rightarrow Z_{\emptyset, D} \text { is }\left(B^{\prime}, B, \tilde{B}, \tilde{B}^{\prime}, g U_{B^{\prime}}\right) \mapsto\left(B^{\prime}, \tilde{B}^{\prime}, g U_{B^{\prime}}\right), \\
& \mathfrak{j}^{\prime}=\mathfrak{j} s^{\prime}: \Upsilon^{\prime} \rightarrow Z_{\emptyset, D} \text { is }\left(B^{\prime}, B, \tilde{B}, \tilde{B}^{\prime}, g U_{B^{\prime}}\right) \mapsto\left(B, \tilde{B}, x U_{B}\right)
\end{aligned}
$$


and $x \in D$ is such that $x B x^{-1}=\tilde{B}, x U_{B^{\prime}}=g U_{B^{\prime}}\left(\right.$ then $x\left(U_{B} \cap U_{B^{\prime}}\right)$ is well defined). We have a commutative diagram with a cartesian right square

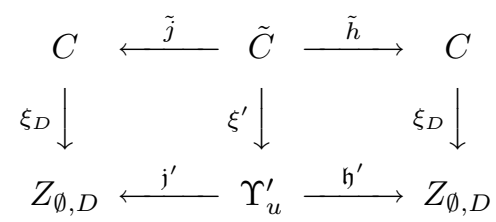

where $\xi_{D}$ is as in 41.1 ,

$$
\begin{aligned}
\tilde{C}=\{ & \left(h_{1} U^{*}, h_{2} B^{*}, h_{3} B^{*}, h_{4} U^{*}\right) \in\left(G^{0} / U^{*}\right)^{4} ; \\
& \left.h_{1}^{-1} h_{2} \in B^{*} \dot{u}^{-1} B^{*}, h_{3}^{-1} h_{4} \in B^{*} \delta \dot{u} \delta^{-1} B^{*}\right\},
\end{aligned}
$$

$\tilde{h}$ is $\left(h_{1} U^{*}, h_{2} B^{*}, h_{3} B^{*}, h_{4} U^{*}\right) \mapsto\left(h_{1} U^{*}, h_{4} U^{*}\right), \xi^{\prime}$ is

$$
\begin{aligned}
& \left(h_{1} U^{*}, h_{2} B^{*}, h_{3} B^{*}, h_{4} U^{*}\right) \\
& \mapsto\left(h_{1} B^{*} h_{1}^{-1}, h_{2} B^{*} h_{2}^{-1}, h_{3} B^{*} h_{3}^{-1}, h_{4} B^{*} h_{4}^{-1}, h_{4} \delta h_{1}^{-1} U_{h_{1} B^{*} h_{1}^{-1}}\right),
\end{aligned}
$$

$\tilde{j}$ is $\left(h_{1} U^{*}, h_{2} B^{*}, h_{3} B^{*}, h_{4} U^{*}\right) \mapsto\left(h_{2} t^{-1} U^{*}, h_{3} \tilde{t} U^{*}\right)$ where $t, \tilde{t} \in T$ are given by $h_{1}^{-1} h_{2} \in U^{*} \dot{u}^{-1} t U^{*}, h_{3}^{-1} h_{4} \in U^{*} \tilde{t} \delta \dot{u} \delta^{-1} U^{*}$.

We see that for $A \in \mathcal{D}\left(Z_{\emptyset, D}\right)$ we have

$$
\xi_{D}^{*} \Phi_{u}(A)=\xi_{D} \mathfrak{h}_{!} \mathfrak{j}^{*} A=\xi_{D}^{*} \mathfrak{h}_{\mathfrak{j}}^{\prime} \mathfrak{j}^{\prime *} A=\tilde{h}_{!} \xi^{\prime *} \mathfrak{j}^{\prime *} A=\tilde{h}_{!} \tilde{j}^{*} \xi_{D}^{*} A
$$

Taking here $A=\underline{\mathcal{L}}_{w}^{\sharp}$ (with $w \in \mathbf{W}, \lambda \in \underline{\mathfrak{s}}, \mathcal{L} \in \lambda$ with $w \underline{D} \lambda=\lambda$ ) and using 41.1(c) we obtain $\xi_{D}^{*} \Phi_{u}\left(\underline{\mathcal{L}}_{w}^{\sharp}\right)=\tilde{h}_{!} \tilde{j}^{*}\left({\underline{\left(\operatorname{Ad}\left(\delta^{-1}\right)^{*} \mathcal{L}\right)^{\sharp}}}_{w}\right)$ or equivalently $\xi_{D}^{*} \Phi_{u}\left(\underline{\dot{\mathcal{L}}}_{w}^{\sharp}\right)=$ $\bar{\sigma}_{!} \tilde{j}^{\prime *}\left(\left(\operatorname{Ad}\left(\delta^{-1}\right) * \mathcal{L}\right)_{w}^{\sharp}\right)$ where

$$
\bar{X}=\left\{\left(h_{1} U^{*}, h_{2} B^{*}, h_{3} B^{*}, h_{4} U^{*}\right) \in \tilde{C} ; h_{2}^{-1} h_{3} \in \overline{B^{*} \dot{w} B^{*}}\right\}
$$

and $\tilde{j}^{\prime}: \bar{X} \rightarrow \bar{C}_{w}, \bar{\sigma}: \bar{X} \rightarrow C$ are the restrictions of $\tilde{j}, \tilde{h}$. Let

$$
\bar{X}_{0}=\left\{\left(h_{1} U^{*}, h_{2} B^{*}, h_{3} B^{*}, h_{4} U^{*}\right) \in \tilde{C} ; h_{2}^{-1} h_{3} \in B^{*} \dot{w} B^{*}\right\}
$$

and let $\tilde{j}_{0}^{\prime}: \bar{X}_{0} \rightarrow C_{w}$ be the restriction of $\tilde{j}$. Let $\mathcal{F}_{0}=\tilde{j}_{0}^{\prime *}\left(\operatorname{Ad}\left(\delta^{-1}\right)^{*} \mathcal{L}\right)$, a local system on $\bar{X}_{0}$. Since $\tilde{j}^{\prime}$ is a fibration with smooth connected fibres, we have $\tilde{j}^{\prime *}\left(\left(\operatorname{Ad}\left(\delta^{-1}\right)^{*} \mathcal{L}\right)_{w}^{\sharp}\right)=I C\left(\bar{X}, \mathcal{F}_{0}\right)$. Thus, $\xi_{D}^{*} \Phi_{u}\left(\underline{\underline{\mathcal{L}}}_{w}^{\sharp}\right)=\bar{\sigma}_{!}\left(I C\left(\bar{X}, \mathcal{F}_{0}\right)\right)$. From the definitions we see that $\mathcal{F}_{0}=\bar{\tau}^{*} \mathcal{L}^{\prime \prime}$, hence $\bar{\sigma}_{!}\left(\operatorname{IC}\left(\bar{X}, \mathcal{F}_{0}\right)\right)=\bar{K}$ and

$$
\xi_{D}^{*} \Phi_{u}\left(\underline{\mathcal{L}}_{w}^{\sharp}\right)=\bar{K}
$$

where $\bar{\tau}^{*} \mathcal{L}^{\prime}, \bar{K}$ are given as in 40.10 in terms of

$$
\left(u^{-1}, \mathcal{L}\right),\left(w, \operatorname{Ad}\left(\delta^{-1}\right)^{*} \mathcal{L}\right),\left(\epsilon(u), \operatorname{Ad}\left(\delta \dot{u} \delta^{-1}\right)^{*} \operatorname{Ad}\left(\delta^{-1}\right)^{*} \mathcal{L}\right)
$$

instead of $(w, \mathcal{L}),\left(w^{\prime}, \mathcal{L}^{\prime}\right),\left(w^{\prime \prime}, \mathcal{L}^{\prime \prime}\right)$.

41.3. For $J \subset \mathbf{I}$ let $\mathcal{D}_{J}^{c s}(C)$ be the subcategory of $\mathcal{D}^{c s}(C)$ whose objects are those $K \in \mathcal{D}(C)$ such that for any $j$, any simple subquotient of ${ }^{p} H^{j} K$ is isomorphic to $\underline{\mathcal{L}}_{w}^{\sharp}$ for some $\mathcal{L} \in \mathfrak{s}$ and some $w \in \mathbf{W}_{J}$.

Let $J, J^{\prime} \subset \mathbf{I}$. Let $K \in \mathcal{D}_{J}^{c s}(C), K^{\prime} \in \mathcal{D}_{J^{\prime}}^{c s}(C)$, and let $w^{\prime}, w^{\prime \prime} \in \mathbf{W}, \lambda^{\prime}, \lambda^{\prime \prime} \in \mathfrak{s}$, $\mathcal{L}^{\prime} \in \lambda^{\prime}, \mathcal{L}^{\prime \prime} \in \lambda^{\prime \prime}$. Let $A=\underline{\mathcal{L}}_{w^{\prime \prime}}^{\prime \prime} \sharp\left[d_{w^{\prime \prime}}\right]$. We show that

(a) if (i) $A \dashv K * \underline{\mathcal{L}}_{w^{\prime}}^{\prime} \sharp\left[d_{w^{\prime}}\right]$ or (ii) $A \dashv \underline{\mathcal{L}}_{w^{\prime}}^{\prime} \sharp\left[d_{w^{\prime}}\right] * K^{\prime}$ or (iii) $A \dashv K * \underline{\mathcal{L}}_{w^{\prime}}^{\prime} \sharp\left[d_{w^{\prime}}\right] * K^{\prime}$, then $\left(w^{\prime \prime}, \lambda^{\prime \prime}\right) \preceq J_{J}\left(w^{\prime}, \lambda^{\prime}\right)$. 
For the proof we may assume that $\mathbf{k}$ is an algebraic closure of a finite field. Then the results in 40.7 are applicable. We first consider the case (i). In this case we can

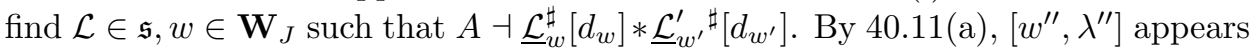
with nonzero coefficient in the expansion of the product $[w, \lambda] *\left[w^{\prime}, \lambda^{\prime}\right]$ in terms of the basis $([y, \nu])$ of $\mathfrak{K}(C)$. Applying $\omega$ (see $40.7(\mathrm{~b})$ ) we see that $c_{w^{\prime \prime}, \lambda^{\prime \prime}}$ appears with nonzero coefficient in the expansion of the product $c_{w, \lambda} c_{w^{\prime}, \lambda^{\prime}}$ in terms of the basis $\left(c_{y, \nu}\right)$ of $H$ and the desired result follows. Case (ii) is treated in an entirely similar way. We now consider case (iii). In this case we must have $A \dashv A^{\prime} * K^{\prime}$ for some simple perverse sheaf $A^{\prime}$ such that $A^{\prime} \dashv K * \underline{\mathcal{L}}_{w^{\prime}}^{\prime} \sharp\left[d_{w^{\prime}}\right]$. We have $A^{\prime}=\underline{\mathcal{M}}_{y}^{\sharp}\left[d_{y}\right]$ where $y \in \mathbf{W}, \mathcal{M} \in \mathfrak{s}$. Let $\nu$ be the isomorphism class of $\mathcal{M}$. From case (ii) applied to $A \dashv A^{\prime} * K^{\prime}$ we see that $\left(w^{\prime \prime}, \lambda^{\prime \prime}\right) \preceq_{J, J^{\prime}}(y, \nu)$. From case (i) applied to $A^{\prime} \dashv K * \underline{\mathcal{L}}_{w^{\prime}}^{\prime} \sharp\left[d_{w^{\prime}}\right]$ we see that $(y, \nu) \preceq J, J^{\prime}\left(w^{\prime}, \lambda^{\prime}\right)$. Combining these two inequalities we obtain $\left(w^{\prime \prime}, \lambda^{\prime \prime}\right) \preceq_{J, J^{\prime}}\left(w^{\prime}, \lambda^{\prime}\right)$, as desired.

41.4. Let $J \subset \mathbf{I}$. In the remainder of this section we write $\mathfrak{f}, \mathfrak{e}$ instead of $\mathfrak{f}_{\emptyset, J}$ : $\mathcal{D}\left(Z_{\emptyset, D} \rightarrow \mathcal{D}\left(Z_{J, D}\right), \mathfrak{e}_{\emptyset, J}: \mathcal{D}\left(Z_{J, D} \rightarrow \mathcal{D}\left(Z_{\emptyset, D}\right)\right.\right.$. We note that

(a) if $A \in \mathcal{D}\left(Z_{J, D}\right)$, then $\mathfrak{f e}(A) \cong A[m] \oplus A^{\prime}$ for some $m \in \mathbf{Z}$ and some $A^{\prime} \in$ $\mathcal{D}\left(Z_{J, D}\right)$.

See $[\mathrm{G}$, $\mathrm{MV}]$ for the special case $D=G^{0}, J=\mathbf{I}$ and $[\mathrm{L} 10,6.6]$ for the general case. Now:

(b) Let $A$ be a simple perverse sheaf on $Z_{J, D}$. Then $A \dashv \mathfrak{f}\left({ }^{p} H^{j}(\mathfrak{e}(A))\right)$ for some $j \in \mathbf{Z}$.

Assume that this is not true. As in BBD, p. 142], for any $n \in \mathbf{Z}$ we have a distinguished triangle $\left({ }^{p} \tau_{\leq n-1} \mathfrak{e} A,{ }^{p} \tau_{\leq n} \mathfrak{e} A,{ }^{p} H^{n}(\mathfrak{e} A)[-n]\right)$, hence a distinguished triangle

$$
\left(\mathfrak{f}\left({ }^{p} \tau_{\leq n-1} \mathfrak{e} A\right), \mathfrak{f}\left({ }^{p} \tau_{\leq n} \mathfrak{e} A\right), \mathfrak{f}\left({ }^{p} H^{n}(\mathfrak{e} A)\right)[-n]\right) .
$$

Using our assumption, we see that $A \dashv \mathfrak{f}\left({ }^{p} \tau_{\leq n-1} \mathfrak{e} A\right)$ if and only if $A \dashv \mathfrak{f}\left({ }^{p} \tau_{\leq n} \mathfrak{e} A\right)$. Thus we have $A \dashv \mathfrak{f}\left({ }^{p} \tau_{\leq n} \mathfrak{e} A\right)$ for some $n$ if and only if $A \dashv \mathfrak{f}\left({ }^{p} \tau_{\leq n} \mathfrak{e} A\right)$ for any $n$. Since ${ }^{p} \tau_{\leq n} \mathfrak{e} A=0$ for some $n$, we see that $A \rtimes \mathfrak{f}\left({ }^{p} \tau_{\leq n} \mathfrak{e} A\right)$ for any $n$. Since ${ }^{p} \tau_{\leq n} \mathfrak{e} A=\mathfrak{e} A$ for some $n$, we deduce that $A \nrightarrow \mathfrak{f} \mathfrak{e} A$. This contradicts (a); (b) is proved.

We show that

(c) if $A$ is a simple perverse sheaf on $Z_{J, D}$, then there exists a simple perverse sheaf $A^{\prime}$ on $Z_{\emptyset, D}$ such that $A \dashv \mathfrak{f}\left(A^{\prime}\right), A^{\prime} \dashv \mathfrak{e}(A)$.

By (b) we can find $i, j \in \mathbf{Z}$ such that $A \dashv{ }^{p} H^{i}(\mathfrak{f}(P))$ where $P={ }^{p} H^{j}(\mathfrak{e}(A))$.

Assume that $A \rtimes^{p} H^{i}\left(\mathfrak{f}\left(A^{\prime}\right)\right)$ for any simple subquotient $A^{\prime}$ of $P$. We claim that $A \rtimes^{p} H^{i}\left(\mathfrak{f}\left(P^{\prime}\right)\right)$ for any subobject $P^{\prime}$ of $P$. We argue by induction on the length of $P^{\prime}$. If $P^{\prime}$ has length 1 , the claim holds by assumption. If $P^{\prime}$ has length $\geq 2$, we can find a simple subobject $P^{\prime \prime}$ of $P^{\prime}$. We have a distinguished triangle $\left(\mathfrak{f}\left(P^{\prime \prime}\right), \mathfrak{f}\left(P^{\prime}\right), \mathfrak{f}\left(P^{\prime} / P^{\prime \prime}\right)\right)$. Hence we have an exact sequence ${ }^{p} H^{i}\left(\mathfrak{f}\left(P^{\prime \prime}\right)\right) \rightarrow$ ${ }^{p} H^{i}\left(\mathfrak{f}\left(P^{\prime}\right)\right) \rightarrow{ }^{p} H^{i}\left(\mathfrak{f}\left(P^{\prime} / P^{\prime \prime}\right)\right)$. By the induction hypothesis, we have $A \bigwedge^{p} H^{i}\left(\mathfrak{f}\left(P^{\prime \prime}\right)\right)$, $A \rtimes^{p} H^{i}\left(\mathfrak{f}\left(P^{\prime} / P^{\prime \prime}\right)\right)$. Hence $A \rtimes^{p} H^{i}\left(\mathfrak{f}\left(P^{\prime}\right)\right)$. This proves the claim. In particular, $A \rtimes^{p} H^{i}(\mathfrak{f}(P))$, contradicting the definition of $i, P$.

We see that there exists a simple subquotient $A^{\prime}$ of $P$ such that $A \dashv{ }^{p} H^{i}\left(\mathfrak{f}\left(A^{\prime}\right)\right)$. Then $A^{\prime}$ is as required by (c).

Let $\bar{d}_{w}=\operatorname{dim} Z_{\emptyset, D}^{w}$. Let

(d) $A^{\prime}=\underline{\dot{\mathcal{L}}}_{w}^{\sharp}\left[\bar{d}_{w}\right], A^{\prime \prime}=\underline{\dot{\mathcal{M}}}_{y}^{\sharp}\left[\bar{d}_{y}\right] \in \hat{Z}_{\emptyset, D}, \mathcal{L} \in \lambda, \mathcal{M} \in \nu$. 
Here $w \underline{D} \lambda=\lambda, y \underline{D} \nu=\nu$. Now:

(e) Let $A$ be a character sheaf on $Z_{J, D}$ such that $A \dashv \mathfrak{f}\left(A^{\prime}\right), A^{\prime \prime} \dashv \mathfrak{e}(A)$. Then $(y, \underline{D} \nu) \preceq_{J, J^{\prime}}(w, \underline{D} \lambda)$.

Since $\mathfrak{f}$ is proper, $\mathfrak{f}\left(A^{\prime}\right)$ is a semisimple complex (see [BBD]). Hence $\mathfrak{f}\left(A^{\prime}\right) \cong A[m] \oplus$ $A_{1}$ for some $m \in \mathbf{Z}, A^{\prime} \in \mathcal{D}\left(Z_{J, D}\right)$ and $\mathfrak{e f}\left(A^{\prime}\right) \cong \mathfrak{e}(A)[m] \oplus \mathfrak{e}\left(A_{1}\right)$. Hence from $A^{\prime \prime} \dashv$ $\mathfrak{e}(A)$ we can deduce $A^{\prime \prime} \dashv \mathfrak{e f}\left(A^{\prime}\right)$. By 37.2 we have $\mathfrak{e f}\left(A^{\prime}\right) \approx\left\{\Phi_{u}\left(A^{\prime}\right)\left[\left[-m_{u}\right]\right] ; u \in\right.$ $\left.\mathbf{W}_{J}\right\}$ where $m_{u}$ are certain integers. Hence for some $u \in \mathbf{W}_{J}$ we have $A^{\prime \prime} \dashv$ $\Phi_{u}\left(A^{\prime}\right)\left[\left[-m_{u}\right]\right]$, that is, $A^{\prime \prime} \dashv \Phi_{u}\left(A^{\prime}\right)$ and $\xi_{D}^{*} A^{\prime \prime}[\mathbf{r}] \dashv \xi_{D}^{*} \Phi_{u}\left(A^{\prime}\right)[\mathbf{r}]$. Hence using 41.2(a) we have $\xi_{D}^{*} A^{\prime \prime}[\mathbf{r}] \dashv \bar{K}$ where $\bar{K}$ is as in the end of 41.2. Thus, $\underline{\mathcal{M}}_{y}^{\sharp}\left[d_{y}\right] \dashv \bar{K}$. Using 40.10(b) we deduce that

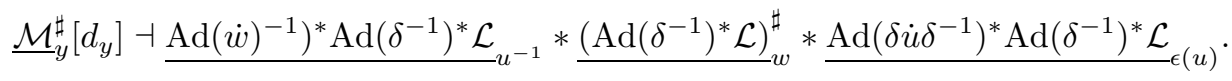

Using this and 41.3(a) we see that (e) holds.

Now:

(f) Let $A$ be a character sheaf on $Z_{J, D}$. In the setup of (d) assume that $A \dashv \mathfrak{f}\left(A^{\prime}\right)$, $A^{\prime} \dashv \mathfrak{e}(A), A \dashv \mathfrak{f}\left(A^{\prime \prime}\right), A^{\prime \prime} \dashv \mathfrak{e}(A)$. Then $(y, \underline{D} \nu) \sim_{J, J^{\prime}}(w, \underline{D} \lambda)$.

Applying (e) to $A^{\prime}, A^{\prime \prime}$ we see that $(y, \underline{D} \nu) \preceq_{J, J^{\prime}}(w, \underline{D} \lambda)$. Applying (e) to $A^{\prime \prime}, A^{\prime}$ (instead of $\left.A^{\prime}, A^{\prime \prime}\right)$ we see that $(w, \underline{D} \lambda) \preceq_{J, J^{\prime}}(y, \underline{D} \nu)$. Hence (f) holds.

From (c),(f) we see that there is a well-defined map $A \mapsto \mathbf{c}_{A}$ from the set of character sheaves on $Z_{J, D}$ (up to isomorphism) to the set of $\left(J, J^{\prime}\right)$-two-sided cells in $\mathbf{W} \times \underline{\mathcal{F}}$ where $\mathbf{c}_{A}$ is the unique $\left(J, J^{\prime}\right)$-two-sided cell that contains

$$
\left\{(w, \underline{D} \lambda) \in \mathbf{W} \times \underline{\mathfrak{s}} ; w \underline{D} \lambda=\lambda, A \dashv \mathfrak{f}\left(\dot{\mathcal{L}}_{w}^{\sharp}\left[\bar{d}_{w}\right]\right), \underline{\mathcal{L}}_{w}^{\sharp}\left[\bar{d}_{w}\right] \dashv A\right\}
$$

(a nonempty set); here $\mathcal{L} \in \lambda$.

41.5. In the setup of 41.4 , let $A$ be a character sheaf on $Z_{J, D}$. We show that:

(a) There exists $(w, \underline{D} \lambda) \in \mathbf{c}_{A}$ such that $w \underline{D} \lambda=\lambda, A \dashv \mathfrak{f}\left(\underline{\dot{\mathcal{L}}}_{w}^{\sharp}\left[\bar{d}_{w}\right]\right)$. If $\left(w^{\prime}, \underline{D} \lambda^{\prime}\right) \in$ $\mathbf{W} \times \underline{\mathfrak{s}}$ is such that $w^{\prime} \underline{D} \lambda^{\prime}=\lambda^{\prime}, A \dashv \mathfrak{f}\left(\underline{\dot{\mathcal{L}}}_{w^{\prime}}^{\prime} \sharp\left[\bar{d}_{w^{\prime}}\right]\right)$, then $(w, \underline{D} \lambda) \preceq{ }_{J, J^{\prime}}\left(w^{\prime}, \underline{D} \lambda^{\prime}\right)$. Here $\mathcal{L} \in \lambda, \mathcal{L}^{\prime} \in \lambda^{\prime}$.

(b) There exists $(w, \underline{D} \lambda) \in \mathbf{c}_{A}$ such that $w \underline{D} \lambda=\lambda, \underline{\dot{\mathcal{L}}}_{w}^{\sharp}\left[\bar{d}_{w}\right] \dashv \mathfrak{e}(A)$. If $\left(w^{\prime}, \underline{D} \lambda^{\prime}\right) \in$ $\mathbf{W} \times \underline{\mathfrak{s}}$ is such that $w^{\prime} \underline{D} \lambda^{\prime}=\lambda^{\prime}, \underline{\dot{\mathcal{L}}}_{w^{\prime}}^{\prime}{ }^{\sharp}\left[\bar{d}_{w^{\prime}}\right] \dashv \mathfrak{e}(A)$, then $\left(w^{\prime}, \underline{D} \lambda^{\prime}\right) \preceq_{J, J^{\prime}}(w, \underline{D} \lambda)$. Here $\overline{\mathcal{L}} \in \lambda, \mathcal{L}^{\prime} \in \lambda^{\prime}$.

Note that (a) follows immediately from $41.4(\mathrm{c})$,(e) and the definition of $\mathbf{c}_{A}$. Similarly, (b) follows from 41.4(c),(e) and the definition of $\mathbf{c}_{A}$.

41.6. In this subsection we assume that $J=\mathbf{I}$. The $\mathcal{A}$ linear map $H \rightarrow H$ given by

(a) $\tilde{T}_{w} 1_{\lambda} \mapsto \tilde{T}_{\epsilon(w)} 1_{\underline{D} \lambda}$ for $w \in \mathbf{W}, \lambda \in \underline{\mathfrak{s}}$

is an $\mathcal{A}$-algebra isomorphism. It carries $c_{w, \lambda}$ to $c_{\epsilon(w), \underline{D} \lambda}$ for any $w \in \mathbf{W}, \lambda \in \underline{\mathfrak{s}}$. It induces a bijection $\mathbf{c} \mapsto \mathbf{c}^{\prime}$ from the set of two-sided cells in $\mathbf{W} \times \mathfrak{s}$ onto itself. We show that

(b) if $A$ is a character sheaf on $D$, then $\left(\mathbf{c}_{A}\right)^{\prime}=\mathbf{c}_{A}$.

Consider the automorphism $\operatorname{Ad}(\delta): D \rightarrow D$. From the definitions we see that for $(w, \lambda) \in \mathbf{W} \times \underline{\mathfrak{s}}$ such that $w \underline{D} \lambda=\lambda$ we have $A \dashv \mathfrak{f}\left(\underline{\mathcal{L}}_{w}^{\sharp}\left[\bar{d}_{w}\right]\right)$ if and only if 
$\operatorname{Ad}\left(\delta^{-1}\right)^{*} A \dashv \mathfrak{f}\left(\underline{\operatorname{Ad}\left(\underline{D}^{-1}\right) * \mathcal{L}_{\epsilon(w)}^{\sharp}}\left[\bar{d}_{w}\right]\right)$. Using this and 41.5(a) we see that

$$
\mathbf{c}_{\mathrm{Ad}\left(\delta^{-1}\right)^{*} A}=\left(\mathbf{c}_{A}\right)^{\prime} \text {. }
$$

It is then enough to show that $\operatorname{Ad}\left(\delta^{-1}\right)^{*} A \cong A$. By the $G^{0}$-equivariance of $A$ we have $m^{*} A \cong q^{*} A$ where $m: G^{0} \times D \rightarrow D$ is $(x, g) \mapsto x g x^{-1}$ and $q: G^{0} \times D \rightarrow D$ is $(x, g) \mapsto g$. Define $r: D \rightarrow G^{0} \times D$ by $r(g)=\left(\delta g^{-1}, g\right)$. Then $r^{*} m^{*} A \cong r^{*} q^{*} A$ that is, $(m r)^{*} A \cong(q r)^{*} A$. We have $m r=\operatorname{Ad}(\delta), q r=1$, hence $\operatorname{Ad}(\delta)^{*} A \cong A$ and $\operatorname{Ad}\left(\delta^{-1}\right)^{*} A \cong A$, as required.

Note also that for $(w, \lambda)$ as above we have

$$
\text { (c) } \mathfrak{f}\left({\underline{\operatorname{Ad}\left(\underline{D}^{-1}\right) * \mathcal{L}_{\epsilon(w)}^{\sharp}}}_{\epsilon}\left[\bar{d}_{w}\right]\right) \cong \mathfrak{f}\left(\underline{\dot{\mathcal{L}}}_{w}^{\sharp}\left[\bar{d}_{w}\right]\right) \text {. }
$$

Indeed, let $K=\mathfrak{f}\left(\underline{\dot{\mathcal{L}}}_{w}^{\sharp}\left[\bar{d}_{w}\right]\right)$. Clearly, we have $m^{*} K \cong q^{*} K$ with $m, q$ as above. Then as in the proof of (b) we see that $\operatorname{Ad}(\delta)^{*} K \cong K$. From the definitions we see that $\mathfrak{f}\left(\underline{\operatorname{Ad}\left(\underline{D}^{-1}\right)^{*} \mathcal{L}_{\epsilon(w)}^{\sharp}}\left[\bar{d}_{w}\right]\right)=\operatorname{Ad}\left(\delta^{-1}\right)^{*} K$. Since $\operatorname{Ad}\left(\delta^{-1}\right)^{*} K \cong K$, (c) follows.

41.7. In this and the next subsection we assume that $\mathbf{k}$ is an algebraic closure of a finite field. From $41.1(\mathrm{c})$ we see that $\xi_{D}^{*}: \mathcal{D}\left(Z_{\emptyset, D}\right) \rightarrow \mathcal{D}(C)$ restricts to a functor $\mathcal{D}^{c s}\left(Z_{\emptyset, D}\right) \rightarrow \mathcal{D}^{c s}(C)$, hence, as in 36.8 , the $\mathcal{A}$-linear map $\operatorname{gr}\left(\xi_{D}^{*}\right): \mathfrak{K}\left(Z_{\emptyset, D}\right) \rightarrow \mathfrak{K}(C)$ is well defined; from $41.1(\mathrm{c})$ we see also that

(a) $\operatorname{gr}\left(\xi_{D}^{*}\right)\left(\dot{\mathcal{L}}_{w}^{\sharp}\left[\bar{d}_{w}\right]\right)=(-v)^{\mathbf{r}}[w ; \underline{D} \lambda]$

for $w \in \mathbf{W}, \lambda \in \underline{\mathfrak{s}}$ such that $w \underline{D} \lambda=\lambda$ and $\mathcal{L} \in \lambda$. From (a) we see that $\operatorname{gr}\left(\xi_{D}^{*}\right)$ is injective with image equal to $\mathfrak{K}(C)^{D}$, the $\mathcal{A}$-submodule of $\mathfrak{K}(C)$ spanned by $\{[w ; \underline{D} \lambda] ; w \in \mathbf{W}, \lambda \in \underline{\mathfrak{s}}, w \underline{D} \lambda=\lambda\}$ or equivalently by $\left\{[w ; \underline{D} \lambda]^{\prime} ; w \in \mathbf{W}, \lambda \in\right.$ $\underline{\mathfrak{s}}, w \underline{D} \lambda=\lambda$. Thus, $\operatorname{gr}\left(\xi_{D}^{*}\right)$ defines an isomorphism $\eta^{\prime}: \mathfrak{K}\left(Z_{\emptyset, D}\right) \stackrel{\sim}{\longrightarrow} \mathfrak{K}(C)^{D}$. Let $\eta=\eta^{\prime-1}$.

Let $n \in \mathbf{N}_{\mathbf{k}}^{*}$. Let $\mathfrak{K}(C)_{n}^{D}$ be the $\mathcal{A}$-submodule of $\mathfrak{K}(C)$ spanned by $\{[w ; \underline{D} \lambda] ; w \in$ $\left.\mathbf{W}, \lambda \in \underline{\mathfrak{s}}_{n}, w \underline{D} \lambda=\lambda\right\}$ or equivalently by $\left\{[w ; \underline{D} \lambda]^{\prime} ; w \in \mathbf{W}, \lambda \in \underline{\mathfrak{s}}_{n}, w \underline{D} \lambda=\lambda\right\}$.

Let $u, w \in \mathbf{W}, \lambda \in \underline{\mathfrak{s}}_{n}$ be such that $w \underline{D} \lambda=\lambda$ and let $\mathcal{L} \in \lambda$. From 37.3(c) we see that the $\mathcal{A}$-linear map $\operatorname{gr}\left(\Phi_{u}\right): \mathfrak{K}\left(Z_{\emptyset, D}\right) \rightarrow \mathfrak{K}\left(Z_{\emptyset, D}\right)$ is well defined; we denote it again by $\Phi_{u}$. From $40.10(a), 41.2($ a) we have

$$
\left[u^{-1} ; \lambda\right]^{\prime} *[w ; \underline{D} \lambda]^{\prime \sharp} *\left[\epsilon_{D}(u) ; \underline{D}\left(u^{-1} \lambda\right)\right]^{\prime}=\left(v^{2}-1\right)^{2 \mathbf{r}} \eta^{\prime} \Phi_{u} \eta\left([w ; \underline{D} \lambda]^{\prime \sharp}\right),
$$

equality in $\mathfrak{K}(C)$. If $\lambda^{\prime} \in \underline{\mathfrak{s}}_{n}, \lambda^{\prime} \neq \lambda$, we have (from 40.7) that $\left[u^{-1} ; \lambda^{\prime}\right]^{\prime} *[w ; \underline{D} \lambda]^{\prime \sharp} *$ $\left[\epsilon_{D}(u) ; \underline{D}\left(u^{-1} \lambda^{\prime}\right)\right]^{\prime}=0$. It follows that

$$
\left.\left(v^{2}-1\right)^{2 \mathbf{r}} \eta^{\prime} \Phi_{u} \eta\left([w, \underline{D} \lambda]^{\prime \sharp}\right)\right)=\sum_{\lambda^{\prime} \in \underline{\mathfrak{s}}_{n}}\left[u^{-1} ; \lambda^{\prime}\right]^{\prime} *[w ; \underline{D} \lambda]^{\prime \sharp} *\left[\epsilon_{D}(u) ; \underline{D}\left(u^{-1} \lambda^{\prime}\right)\right]^{\prime} .
$$

Using this and the definition of $\mathfrak{K}(C)_{n}^{D}$ we see that

$$
\left(v^{2}-1\right)^{2 \mathbf{r}} \eta^{\prime} \Phi_{u} \eta(x)=\sum_{\lambda^{\prime} \in \underline{\underline{s}}_{n}}\left[u^{-1} ; \lambda^{\prime}\right]^{\prime} * x *\left[\epsilon_{D}(u) ; \underline{D}\left(u^{-1} \lambda^{\prime}\right)\right]^{\prime}
$$

for any $x \in \mathfrak{K}(C)_{n}^{D}$. Applying $\eta$ to both sides we obtain

$$
\left(v^{2}-1\right)^{2 \mathbf{r}} \Phi_{u} \eta^{\prime}(x)=\sum_{\lambda^{\prime} \in \underline{\mathfrak{s}}_{n}} \eta\left(\left[u^{-1} ; \lambda^{\prime}\right]^{\prime} * x *\left[\epsilon_{D}(u) ; \underline{D}\left(u^{-1} \lambda^{\prime}\right)\right]^{\prime}\right)
$$

for any $x \in \mathfrak{K}(C)_{n}^{D}$. 
41.8. In the setup of 41.4, let $A$ be a character sheaf on $Z_{J, D}$. From 36.9 (b) we see that the condition that, if $\left(w^{\prime}, \underline{D} \lambda^{\prime}\right) \in \mathbf{W} \times \underline{\mathfrak{s}}$ is such that $w^{\prime} \underline{D} \lambda^{\prime}=\lambda^{\prime}$, then we have $A \dashv \mathfrak{f}\left(\underline{\dot{\mathcal{L}}}_{w^{\prime}}^{\prime}{ }^{\prime}\left[\bar{d}_{w^{\prime}}\right]\right)$ if and only if $A$ appears with coefficient $\neq 0$ in the expansion of $\mathfrak{f}\left(\underline{\mathcal{L}}_{w^{\prime}}^{\prime} \sharp\left[\bar{d}_{w^{\prime}}\right]\right) \in \mathfrak{K}\left(Z_{J, D}\right)$ as a linear combination of the canonical basis of $\mathfrak{K}\left(Z_{J, D}\right)$. Hence from $41.5($ a) we deduce:

(a) There exists $(w, \underline{D} \lambda) \in \mathbf{c}_{A}$ such that $w \underline{D} \lambda=\lambda$ and $A$ appears with nonzero coefficient in $\mathfrak{f}\left(\underline{\mathcal{L}}_{w}^{\sharp}\left[\bar{d}_{w}\right]\right) \in \mathfrak{K}\left(Z_{J, D}\right)$. If $\left(w^{\prime}, \underline{D} \lambda^{\prime}\right) \in \mathbf{W} \times \underline{\mathfrak{s}}$ is such that $w^{\prime} \underline{D} \lambda^{\prime}=\lambda^{\prime}$ and $A$ appears with nonzero coefficient in $\mathfrak{f}\left(\underline{\dot{\mathcal{L}}}_{w^{\prime}}^{\prime}{ }^{\sharp}\left[\bar{d}_{w^{\prime}}\right]\right) \in \mathfrak{K}\left(Z_{J, D}\right)$, then $(w, \underline{D} \lambda) \preceq_{J, J^{\prime}}$ $\left(w^{\prime}, \underline{D} \lambda^{\prime}\right)$. Here $\mathcal{L} \in \lambda, \mathcal{L}^{\prime} \in \lambda^{\prime}$.

Clearly, property (a) characterizes $\mathbf{c}_{A}$.

41.9. Let $J \subset J^{\prime} \subset \mathbf{I}$ and let $D^{\prime}$ be another connected component of $G$. Let $A_{0} \in \mathcal{D}\left(Z_{J, D}\right), A^{\prime} \in \mathcal{D}\left(Z_{\epsilon_{D}\left(J^{\prime}\right), D^{\prime}}\right)$. We show that

(a) $\mathfrak{f}_{J, J^{\prime}}\left(A_{0}\right) * A^{\prime} \cong \mathfrak{f}_{J, J^{\prime}}\left(A_{0} * \mathfrak{e}_{\epsilon_{D}(J), \epsilon_{D}\left(J^{\prime}\right)} A^{\prime}\right)$ in $\mathcal{D}\left(Z_{J^{\prime}, D^{\prime} D}\right)$.

Indeed, from the definitions we see that both sides of (a) can be identified with $b_{!} c^{*}\left(A_{0} \otimes A^{\prime}\right)$ where $b, c$ are as in the diagram

$$
Z_{J, D} \times Z_{\epsilon_{D}\left(J^{\prime}\right), D^{\prime}} \stackrel{c}{\leftarrow} Y \stackrel{b}{\rightarrow} Z_{J^{\prime}, D^{\prime} D}
$$

where

$$
\begin{aligned}
Y=\{ & \left(P, R, R^{\prime}, g U_{R}, g^{\prime} U_{R^{\prime}}\right) ; P \in \mathcal{P}_{J}, R \in \mathcal{P}_{J^{\prime}}, R^{\prime} \in \mathcal{P}_{\epsilon_{D}\left(J^{\prime}\right)}, \\
& \left.g U_{R} \in D / U_{R}, g^{\prime} U_{R^{\prime}} \in D^{\prime} / U_{R^{\prime}}, g R g^{-1}=R^{\prime}, P \subset R\right\},
\end{aligned}
$$

$c$ is $\left(P, R, R^{\prime}, g U_{R}, g^{\prime} U_{R^{\prime}}\right) \mapsto\left(\left(P, g U_{P}\right),\left(R^{\prime}, g^{\prime} U_{R^{\prime}}\right), b\right.$ is $\left(P, R, R^{\prime}, g U_{R}, g^{\prime} U_{R^{\prime}}\right) \mapsto$ $\left(R, g^{\prime} g U_{R}\right)$.

An entirely similar proof shows that, if $A \in \mathcal{D}\left(Z_{J^{\prime}, D}\right), A_{0}^{\prime} \in \mathcal{D}\left(Z_{\epsilon_{D}(J), D^{\prime}}\right)$, then

(b) $A * \mathfrak{f}_{\epsilon_{D}(J), \epsilon_{D}\left(J^{\prime}\right)}\left(A_{0}^{\prime}\right) \cong \mathfrak{f}_{J, J^{\prime}}\left(\mathfrak{e}_{J, J^{\prime}} A * A_{0}^{\prime}\right)$ in $\mathcal{D}\left(Z_{J^{\prime}, D^{\prime} D}\right)$.

41.10. Let $\mathbf{c}$ be a two-sided cell in $\mathbf{W} \times \underline{\mathfrak{s}}$. Let $\overline{\mathbf{c}}$ be the set of all $(w, \lambda) \in \mathbf{W} \times \underline{\mathfrak{s}}$ such that $(w, \lambda) \preceq_{\mathbf{I}, \mathbf{I}}(y, \nu)$ for some/any $(y, \nu) \in \mathbf{c}$.

If $K \in \mathcal{D}\left(Z_{\emptyset, D}\right)$, we say that $K \in \mathcal{D}_{\overline{\mathbf{c}}}^{c s}\left(Z_{\emptyset, D}\right)$ if for any $j \in \mathbf{Z}$ and simple subquotient $A$ of ${ }^{p} H^{j}(K)$ satisfies $\mathbf{c}_{A} \subset \overline{\mathbf{c}}$.

Let $D^{\prime}$ be another connected component of $G$. We show that

(a) if $K \in \mathcal{D}_{\overline{\mathbf{c}}}^{c s}\left(Z_{\emptyset, D}\right), K^{\prime} \in \mathcal{D}^{c s}\left(Z_{\epsilon_{D}\left(J^{\prime}\right), D^{\prime}}\right)$, then $K * K^{\prime} \in \mathcal{D}_{\overline{\mathbf{c}}}^{c s}\left(Z_{\emptyset, D^{\prime} D}\right)$.

We may assume that $\mathbf{k}$ is an algebraic closure of a finite field. We may assume that $K \in \hat{Z}_{\emptyset, D}$ and $\mathbf{c}_{K} \subset \overline{\mathbf{c}}$. Then there exists $(w, \underline{D} \lambda) \in \mathbf{c}_{K}$ such that $w \underline{D} \lambda=\lambda$, $K \dashv \mathfrak{f}\left(\underline{\mathcal{L}}_{w}^{\sharp}\left[\bar{d}_{w}\right]\right), \mathcal{L} \in \lambda$. It is enough to show that, if $\tilde{A} \in \hat{Z}_{\emptyset, D^{\prime} D}$ is such that $\tilde{A} \dashv K * K^{\prime}$, then $\mathbf{c}_{\tilde{A}} \subset \overline{\mathbf{c}}$. Since $\mathfrak{f}\left(\underline{\mathcal{L}}_{w}^{\sharp}\left[\bar{d}_{w}\right]\right)$ is a semisimple complex (see the line after $41.4(\mathrm{e}))$ we have $\mathfrak{f}\left(\underline{\mathcal{L}}_{w}^{\sharp}\left[\bar{d}_{w}\right]\right) \cong K[m] \oplus \tilde{K}$ for some $m \in \mathbf{Z}, \tilde{K} \in \mathcal{D}\left(Z_{\emptyset, D^{\prime} D}\right)$. It follows that $\mathfrak{f}\left(\underline{\dot{\mathcal{L}}}_{w}^{\sharp}\left[\bar{d}_{w}\right]\right) * K^{\prime} \cong K * K^{\prime}[m] \oplus \tilde{K} * K^{\prime}$ hence $\tilde{A} \dashv \mathfrak{f}\left(\underline{\mathcal{L}}_{w}^{\sharp}\left[\bar{d}_{w}\right]\right) * K^{\prime}$. By 41.9 (a) we have $\mathfrak{f}\left(\underline{\mathcal{L}}_{w}^{\sharp}\left[\bar{d}_{w}\right]\right) * K^{\prime} \cong \mathfrak{f}\left(\dot{\mathcal{L}}_{w}^{\sharp}\left[\bar{d}_{w}\right] * \mathfrak{e}\left(K^{\prime}\right)\right)$ hence $\tilde{A} \dashv \mathfrak{f}\left(\underline{\mathcal{L}}_{w}^{\sharp}\left[\bar{d}_{w}\right] * \mathfrak{e}\left(K^{\prime}\right)\right)$. We deduce that there exists $K_{0}^{\prime} \in \hat{Z}_{\emptyset, D^{\prime}}$ such that $\tilde{A} \dashv \mathfrak{f}\left(\underline{\dot{\mathcal{L}}}_{w}^{\sharp}\left[\bar{d}_{w}\right] * K_{0}^{\prime}\right)$ and $K_{0}^{\prime \prime} \in$ $\hat{Z}_{\emptyset, D^{\prime} D}$ such that $K_{0}^{\prime \prime} \dashv \underline{\mathcal{L}}_{w}^{\sharp}\left[\bar{d}_{w}\right] * K_{0}^{\prime}, \tilde{A} \dashv \mathfrak{f}\left(K_{0}^{\prime \prime}\right)$. We then have $\xi_{D^{\prime} D}^{*} K_{0}^{\prime \prime}[\mathbf{r}] \dashv$ $\xi_{D^{\prime} D}^{*}\left(\underline{\mathcal{L}}_{w}^{\sharp}\left[\bar{d}_{w}\right] * K_{0}^{\prime}\right)[\mathbf{r}]$, hence, using 41.1(d), $\left.\xi_{D^{\prime} D}^{*} K_{0}^{\prime \prime}[\mathbf{r}] \dashv\left(\xi_{D}^{*} \dot{\mathcal{L}}_{w}^{\sharp}\left[\bar{d}_{w}\right]\right) * \xi_{D^{\prime}} K_{0}^{\prime}\right)$. Setting $g r\left(\xi_{D^{\prime} D}^{*} K_{0}^{\prime \prime}[\mathbf{r}]\right)=\left[w_{1}, \underline{D^{\prime}} \underline{D} \lambda_{1}\right] \in \mathfrak{K}(C)$ with $\left(w_{1}, \lambda_{1}\right) \in \mathbf{W} \times \underline{\mathfrak{s}}$ we see, using 41.3(a) that $\left(w_{1}, \underline{D^{\prime}} \underline{D} \lambda_{1}\right) \preceq_{\mathbf{I}, \mathbf{I}}(w, \underline{D} \lambda)$. From $\tilde{A} \dashv \mathfrak{f}\left(K_{0}^{\prime \prime}\right)$ we see using 41.5 (a) that 
$\mathbf{c}_{\tilde{A}} \preceq_{\mathbf{I}, \mathbf{I}}\left(w_{1}, \underline{D^{\prime}} \underline{D} \lambda_{1}\right)$ (that is, some/any element of $\mathbf{c}_{\tilde{A}}$ is $\preceq_{\mathbf{I}, \mathbf{I}}\left(w_{1}, \underline{D^{\prime}} \underline{D} \lambda_{1}\right)$ ). Using the transitivity of $\preceq_{\mathbf{I}, \mathbf{I}}$ we see that $\mathbf{c}_{\tilde{A}} \preceq_{\mathbf{I}, \mathbf{I}}(w, \underline{D} \lambda)$. This proves (a).

An entirely similar argument shows that

(b) if $K \in \mathcal{D}^{c s}\left(Z_{\emptyset, D}\right), K^{\prime} \in \mathcal{D}_{\overline{\mathbf{c}}}^{c s}\left(Z_{\epsilon_{D}\left(J^{\prime}\right), D^{\prime}}\right)$, then $K * K^{\prime} \in \mathcal{D}_{\overline{\mathbf{c}}}^{c s}\left(Z_{\emptyset, D^{\prime} D}\right)$.

\section{Duality and the Functor $\mathfrak{f}_{\emptyset, \mathbf{I}}$}

42.1. In this section we fix a connected component $D$ of $G$. We write $\epsilon$ instead of $\epsilon_{D}: \mathbf{W} \rightarrow \mathbf{W}$. We write $\mathfrak{f}$ instead of $\mathfrak{f}_{\emptyset, \mathbf{I}}: \mathcal{D}\left(Z_{\emptyset, D} \rightarrow \mathcal{D}\left(Z_{\mathbf{I}, D}\right)\right.$. We assume that $\mathbf{k}$ is an algebraic closure of a finite field.

Let $J \subset \mathbf{I}$ be such that $\epsilon(J)=J$. Recall from 30.3 that $V_{J, D}=\left\{\left(P, g U_{P}\right) ; P \in\right.$ $\left.\mathcal{P}_{J}, g U_{P} \in N_{D} P / U_{P}\right\}$. As in 30.4 (with $J^{\prime}=\mathbf{I}$ ) we consider the diagram $V_{J, D} \stackrel{c}{\leftarrow}$ $V_{J, \mathbf{I}, D} \stackrel{d}{\rightarrow} D$ where $V_{J, \mathbf{I}, D}=\left\{(P, g) ; P \in \mathcal{P}_{J}, g \in N_{D} P\right\}, c$ is $(P, g) \mapsto\left(P, g U_{P}\right)$ and $d$ is $(P, g) \mapsto g$. Define $\tilde{f}_{J}: \mathcal{D}\left(V_{J, D}\right) \rightarrow \mathcal{D}(D), \tilde{e}_{J}: \mathcal{D}(D) \rightarrow \mathcal{D}\left(V_{J, D}\right)$ by $\tilde{f}_{J} A=d_{!} c^{*} A, \tilde{e}_{J} A^{\prime}=c_{!} d^{*} A^{\prime}$. (In the notation of 30.4 we have $\tilde{f}_{J}=\tilde{f}_{J, \mathbf{I}}, \tilde{e}_{J}=\tilde{e}_{J, \mathbf{I}}$.) Define $f_{J}: \mathcal{D}\left(V_{J, D}\right) \rightarrow \mathcal{D}(D), e_{J}: \mathcal{D}(D) \rightarrow \mathcal{D}\left(V_{J, D}\right)$ by $f_{J} A=\tilde{f}_{J} A\left[\left[\alpha_{J} / 2\right]\right]$, $e_{J} A=\tilde{e}_{J} A\left[\left[\alpha_{J} / 2\right]\right]$ where $\alpha_{J}=\operatorname{dim} \mathcal{P}_{J}$. (In the notation of 30.4 we have $f_{J} A=$ $f_{J, \mathbf{I}} A\left(\alpha_{J} / 2\right), e_{J} A=e_{J, \mathbf{I}} A\left(-\alpha_{J} / 2\right)$. Thus, $f_{J}, e_{J}$ are the same, up to a twist, as $\left.f_{J, \mathbf{I}}, e_{J, \mathbf{I} \cdot}\right)$

From 30.5 (with $J^{\prime}=\mathbf{I}$ ) we see that for $A \in \mathcal{D}\left(V_{J, D}\right), A^{\prime} \in \mathcal{D}(D)$ we have canonically

(a) $\operatorname{Hom}_{\mathcal{D}\left(V_{J, D}\right)}\left(e_{J} A^{\prime}, A\right)=\operatorname{Hom}_{\mathcal{D}(D)}\left(A^{\prime}, f_{J} A\right)$.

Let $C S\left(V_{J, D}\right), C S(D)$ be as in 38.1. From 38.2, 38.3 we see that

(b) $f_{J}, e_{J}$ restrict to functors $C S\left(V_{J, D}\right) \rightarrow C S(D), C S(D) \rightarrow C S\left(V_{J, D}\right)$ denoted again by $f_{J}, e_{J}$.

We show that

(c) if $A \in C S\left(V_{J, D}\right)$ comes from a pure complex of weight 0 with respect to a rational structure over a finite subfield of $\mathbf{k}$, then $f_{J} A$ (naturally regarded as a mixed complex) is pure of weight 0.

Indeed, the functor $c^{*}$ preserves pure complexes of weight 0 since $c$ is smooth with connected fibres; the functor $d_{\text {! }}$ preserves pure complexes of weight 0 since $d$ is proper (see [De, 6.2.6]) and $\left[\left[\alpha_{J} / 2\right]\right]$ also preserves pure complexes of weight 0.

We show that

(d) if $A^{\prime} \in C S(D)$ comes from a pure complex of weight 0 with respect to a rational structure over a finite subfield of $\mathbf{k}$, then $e_{J} A^{\prime}$ (naturally regarded as a mixed complex) is pure of weight 0.

Using (b), it is enough to show that for any simple $A$ as in (c), the natural action of Frobenius on the vector space $\operatorname{Hom}_{\mathcal{D}\left(V_{J, D}\right)}\left(e_{J} A^{\prime}, A\right)$ has weight 0 . Using (a) we see that it is enough to show that the natural action of Frobenius on the vector space $\operatorname{Hom}_{\mathcal{D}(D)}\left(A^{\prime}, f_{J} A\right)$ has weight 0 . This follows from (c) using (b).

Define an imbedding $s: V_{J, D} \rightarrow Z_{J, D}$ by $\left(P, g U_{P}\right) \mapsto\left(P, P, g U_{P}\right)$. From the definitions we see that

(e) $\tilde{f}_{J}: \mathcal{D}\left(V_{J, D}\right) \rightarrow \mathcal{D}(D)$ is the composition $\mathcal{D}\left(V_{J, D}\right) \stackrel{s !}{\longrightarrow} \mathcal{D}\left(Z_{J, D}\right) \stackrel{\mathfrak{f}_{J, \mathbf{I}}}{\longrightarrow} \mathcal{D}(D)$,

(f) $\tilde{e}_{J}: \mathcal{D}(D) \rightarrow \mathcal{D}\left(V_{J, D}\right)$ is the composition $\mathcal{D}(D) \stackrel{\mathfrak{e}_{J, \mathbf{I}}}{\longrightarrow} \mathcal{D}\left(Z_{J, D}\right) \stackrel{s^{*}}{\longrightarrow} \mathcal{D}\left(V_{J, D}\right)$.

Let $Y=\left\{\left(B, B^{\prime}, g U_{B}\right) \in Z_{\emptyset, D} ; \operatorname{pos}\left(B, B^{\prime}\right) \in \mathbf{W}_{J}\right\}$ and let $r: Y \rightarrow Z_{\emptyset, D}$ be the inclusion. From the definitions we have

(g) $s ! s^{*} \mathfrak{f}_{\emptyset, J}=\mathfrak{f}_{\emptyset, J} r_{!} r^{*}: \mathcal{D}\left(Z_{\emptyset, D}\right) \rightarrow \mathcal{D}\left(Z_{J, D}\right)$. 
Note that $V_{J, D}={ }^{1} Z_{J, D}$ (see 36.2); hence the "character sheaves" on $V_{J, D}={ }^{1} Z_{J, D}$ are defined as in 36.8 and $\mathcal{D}^{c s}\left(V_{J, D}=\mathcal{D}^{c s}\left({ }^{1} Z_{J, D}\right)\right)$ is defined as 36.8. In particular, $\mathfrak{K}\left(V_{J, D}\right)=\mathfrak{K}\left({ }^{1} Z_{J, D}\right)$ is defined. Let $\mathfrak{K}_{0}\left(V_{J, D}\right)=\bigoplus_{A} \mathbf{Z} A \subset \mathfrak{K}\left(V_{J, D}\right)$ where $A$ runs through the character sheaves on $V_{J, D}$ (up to isomorphism).

From (b) we see that $\tilde{f}_{J}, \tilde{e}_{J}$ restrict to functors $\mathcal{D}^{c s}\left(V_{J, D}\right) \rightarrow \mathcal{D}^{c s}(D), \mathcal{D}^{c s}(D) \rightarrow$ $\mathcal{D}^{c s}\left(V_{J, D}\right)$, hence the $\mathcal{A}$-linear maps $\operatorname{gr}\left(\tilde{f}_{J}\right): \mathfrak{K}\left(V_{J, D}\right) \rightarrow \mathfrak{K}(D), \operatorname{gr}\left(\tilde{e}_{J}\right): \mathfrak{K}(D) \rightarrow$ $\mathfrak{K}\left(V_{J, D}\right)$ are well defined; we denote them by $\tilde{f}_{J}, \tilde{e}_{J}$. Define $f_{J}: \mathfrak{K}\left(V_{J, D}\right) \rightarrow \mathfrak{K}(D)$ by $f_{J}=(-v)^{-\alpha_{J}} \tilde{f}_{J}$ and $e_{J}: \mathfrak{K}(D) \rightarrow \mathfrak{K}\left(V_{J, D}\right)$ by $e_{J}=(-v)^{-\alpha_{J}} \tilde{e}_{J}$. We show that

$(\mathrm{h}) f_{J}: \mathfrak{K}\left(V_{J, D}\right) \rightarrow \mathfrak{K}(D), e_{J}: \mathfrak{K}(D) \rightarrow \mathfrak{K}\left(V_{J, D}\right)$ restrict to group homomorphisms $\mathfrak{K}_{0}\left(V_{J, D}\right) \rightarrow \mathfrak{K}_{0}(D), \mathfrak{K}_{0}(D) \rightarrow \mathfrak{K}_{0}\left(V_{J, D}\right)$ denoted again by $f_{J}, e_{J}$.

It is enough to prove the following statement. If $x$ is a canonical basis element of $\mathfrak{K}\left(V_{J, D}\right)$ (resp. $\mathfrak{K}(D)$ ), then $f_{J}(x)$ (resp. $e_{J}(x)$ ) is an $\mathbf{N}$-linear combination of canonical basis elements of $\mathfrak{K}(D)$ (resp. $\mathfrak{K}\left(V_{J, D}\right)$ ). This is immediate from (c), (d).

Now, one checks easily that $r ! r^{*}: \mathcal{D}\left(Z_{\emptyset, D}\right) \rightarrow \mathcal{D}\left(Z_{\emptyset, D}\right)$ restricts to a functor $\mathcal{D}^{c s}\left(Z_{\emptyset, D}\right) \rightarrow \mathcal{D}^{c s}\left(Z_{\emptyset, D}\right)$. (Note that, if $w \in \mathbf{W}, \lambda \in \underline{\mathfrak{s}}, \mathcal{L} \in \lambda$ and $w \underline{D} \lambda=\lambda$, then $r ! r^{*}\left(\underline{\mathcal{L}}_{w}\right)=\dot{\mathcal{L}}_{w}$ for $w \in \mathbf{W}_{J}$ and $r ! r^{*}\left(\underline{\mathcal{L}}_{w}\right)=0$ for $\left.w \in \mathbf{W}-\mathbf{W}_{J}.\right)$ It follows that the $\mathcal{A}$-linear map $\operatorname{gr}\left(r_{!} r^{*}\right): \mathfrak{K}\left(Z_{\emptyset, D}\right) \rightarrow \mathfrak{K}\left(Z_{\emptyset, D}\right)$ (denoted by $\rho_{J}$ ) is well defined.

Let $\mathfrak{K}(C)^{D}, \eta$ be as in 41.7. Define an $\mathcal{A}$-linear map $\tilde{\rho}_{J}: \mathfrak{K}(C)^{D} \rightarrow \mathfrak{K}(C)^{D}$ by $[w ; \underline{D} \lambda]^{\prime} \mapsto[w ; \underline{D} \lambda]^{\prime}$ if $w \in \mathbf{W}_{J}, \lambda \in \underline{\mathfrak{s}}, w \underline{D} \lambda=\lambda$ and $[w ; \underline{D} \lambda]^{\prime} \mapsto 0$ if $w \in$ $\mathbf{W}-\mathbf{W}_{J}, \lambda \in \underline{\mathfrak{s}}, w \underline{D} \lambda=\lambda$. From the definitions we see that

(i) $\rho_{J} \eta(x)=\eta \tilde{\rho}_{J}(x)$ for all $x \in \mathfrak{K}(C)^{D}$.

42.2. We define an $\mathcal{A}$-linear map $\mathbf{d}: \mathfrak{K}(D) \rightarrow \mathfrak{K}(D)$ by

$$
\mathbf{d}(x)=\sum_{J ; J \subset \mathbf{I} ; \epsilon(J)=J}(-1)^{\left|J_{\epsilon}\right|} f_{J} e_{J}(x)
$$

where $f_{J}, e_{J}$ are as in $42.1(\mathrm{~h})$ and $J_{\epsilon}$ is as in 38.1. Now:

(a) Let $A$ be a character sheaf on $D$. Then $\mathbf{d}(A)= \pm A^{\prime}$ where $A^{\prime}$ is a character sheaf on D. Moreover, \pm and $A^{\prime}$ are the same as in 38.11(a).

For any $J \subset \mathbf{I}$ such that $\epsilon(J)=J$ let $\mathcal{K}\left(V_{J, D}\right)$ be as in 38.9. We shall identify $\mathfrak{K}\left(V_{J, D}\right) /(v-1) \mathfrak{K}\left(V_{J, D}\right)=\mathcal{K}\left(V_{J, D}\right)$ as abelian groups in such a way that the image of $A_{1}$ (a character sheaf on $\left.V_{J, D}\right)$ in $\mathfrak{K}\left(V_{J, D}\right) /(v-1) \mathfrak{K}\left(V_{J, D}\right)$ is identified with the basis element $A_{1}$ of $\mathcal{K}\left(V_{J, D}\right)$. From the definitions we see that the homomorphisms

$$
\mathfrak{K}(D) /(v-1) \mathfrak{K}(D) \rightarrow \mathfrak{K}\left(V_{J, D}\right) /(v-1) \mathfrak{K}\left(V_{J, D}\right) \rightarrow \mathfrak{K}(D) /(v-1) \mathfrak{K}(D)
$$

induced by $e_{J}, f_{J}$ in $42.1(\mathrm{~h})$ are then identified with the homomorphisms

$$
e_{J, \mathbf{I}}: \mathcal{K}(D) \rightarrow \mathcal{K}\left(V_{J, D}\right), f_{J, \mathbf{I}}: \mathcal{K}\left(V_{J, D}\right) \rightarrow \mathcal{K}(D)
$$

in 38.2, 38.3. It follows that the endomorphism of $\mathfrak{K}(D) /(v-1) \mathfrak{K}(D)$ induced by $\mathbf{d}: \mathfrak{K}(D) \rightarrow \mathfrak{K}(D)$ is identified with the homomorphism $\mathcal{K}(D) \rightarrow \mathcal{K}(D)$ denoted in 38.10 (a), 38.11 again by $\mathbf{d}$. Hence we have $\mathbf{d}(A)= \pm A^{\prime}+(v-1) x$ (in $\left.\mathfrak{K}(D)\right)$ where , $\pm A^{\prime}$ are as in 38.11(a) and $x \in \mathfrak{K}(D)$. From $42.1(\mathrm{~h})$ we see that $\mathbf{d}(A) \in \mathfrak{K}_{0}(D)$. Since $\pm A^{\prime} \in \mathfrak{K}_{0}(D)$, we see that $(v-1) x \in \mathfrak{K}_{0}(D)$. Since $\mathfrak{K}_{0}(D) \cap(v-1) \mathfrak{K}(D)=0$, we have $(v-1) x=0$ and $x=0$. This proves (a). 
42.3. We have $H=H_{D} \oplus H_{D}^{\prime}$ where $H_{D}$ (resp. $H_{D}^{\prime}$ ) is the $\mathcal{A}$-submodule of $H_{n}$ spanned by $\left\{\tilde{T}_{w} 1_{\underline{D} \lambda} ; w \in \mathbf{W}, \lambda \in \underline{\mathfrak{s}}, w \underline{D} \lambda=\lambda\right\}$ (resp. by $\left\{\tilde{T}_{w} 1_{\underline{D} \lambda} ; w \in \mathbf{W}, \lambda \in\right.$ $\underline{\mathfrak{s}}, w \underline{D} \lambda \neq \lambda\})$. Equivalently,

$$
H_{D}=\sum_{\lambda \in \underline{\mathfrak{s}}} 1_{\lambda} H 1_{\underline{D} \lambda} \subset H, H_{D}^{\prime}=\sum_{\lambda, \lambda^{\prime} \in \underline{\mathfrak{s}} ; \lambda \neq \lambda^{\prime}} 1_{\lambda^{\prime}} H 1_{\underline{D} \lambda} \subset H .
$$

Recall that $\omega: \mathfrak{K}(C) \stackrel{\sim}{\longrightarrow} H$ is defined in 40.7 (b). Define an $\mathcal{A}$-linear map $\tilde{\omega}: H \rightarrow \mathfrak{K}(C)^{D}$ by

$$
\begin{aligned}
& \tilde{\omega}(y)=\omega^{-1}(y) \text { if } y \in H_{D}, \\
& \tilde{\omega}(y)=0 \text { if } y \in H_{D}^{\prime} .
\end{aligned}
$$

Then $\eta \tilde{\omega}(y) \in \mathfrak{K}\left(Z_{\emptyset, D}\right)$ is well defined for any $y \in H$. Here $\eta$ is as in 41.7.

Let $n \in \mathbf{N}_{\mathbf{k}}^{*}$. Let $H_{n, D}=H_{D} \cap H_{n}$. Note that $H_{n, D}$ is the $\mathcal{A}$-submodule of $H_{n}$ spanned by $\left\{\tilde{T}_{w} 1_{\underline{D} \lambda} ; w \in \mathbf{W}, \lambda \in \underline{\mathfrak{s}}_{n}, w \underline{D} \lambda=\lambda\right\}$.

For $J \subset \mathbf{I}$ such that $\epsilon(J)=J$ we define an $\mathcal{A}$-linear map $\rho_{J, n}: H_{n, D} \rightarrow H_{n, D}$ by

$$
\begin{aligned}
& \tilde{T}_{w} 1_{\underline{D} \lambda} \mapsto \tilde{T}_{w} 1_{\underline{D} \lambda} \text { if } w \in \mathbf{W}_{J}, \lambda \in \underline{\mathfrak{s}}_{n}, w \underline{D} \lambda=\lambda, \\
& \tilde{T}_{w} 1_{\underline{D} \lambda} \mapsto 0 \text { if } w \in \mathbf{W}-\mathbf{W}_{J}, \lambda \in \underline{\mathfrak{s}}_{n}, w \underline{D} \lambda=\lambda .
\end{aligned}
$$

We have the following result.

Lemma 42.4. For any $y \in H_{n, D}$ we have $\mathbf{d}(\mathfrak{f} \eta \tilde{\omega}(y))=\mathfrak{f} \eta \tilde{\omega}(\delta(y))$ where

$$
\delta=\sum_{J \subset \mathbf{I} ; \epsilon(J)=J}(-1)^{\left|J_{\epsilon}\right|} \delta_{J}
$$

with $\delta_{J}: H_{n, D} \rightarrow H_{n, D}$ given by

$$
\delta_{J}(y)=\rho_{J, n}\left(\sum_{u \in \mathbf{W}^{J}} \tilde{T}_{u^{-1}} y \tilde{T}_{\epsilon_{D}(u)}\right)
$$

(the sum in the right-hand side is computed in $H_{n}$ but it belongs to $H_{n, D}$ ).

Applying 37.2 with $K, K^{\prime}, J$ repaced by $\emptyset, J, \mathbf{I}$ and with $A^{\prime} \in \mathcal{D}^{c s}\left(Z_{\emptyset, D}\right)$ we obtain

$$
\mathfrak{e}_{J, \mathbf{I}} \mathfrak{f} A^{\prime} \approx\left\{\mathfrak{f}_{\emptyset, J} \Phi_{u} A^{\prime}\left[\left[-m_{u}\right]\right] ; u \in \mathbf{W}^{J}\right\}
$$

(in $\mathcal{D}\left(Z_{J, D}\right)$, with $\Phi_{u}: \mathcal{D}\left(Z_{\emptyset, D}\right) \rightarrow \mathcal{D}\left(Z_{\emptyset, D}\right)$ as in 37.1 and $m_{u}=\alpha_{J}-\lambda(u)$ where $\alpha_{J}=\operatorname{dim} \mathcal{P}_{J}$. Applying here $s^{*}$ we obtain

$$
s^{*} \mathfrak{e}_{J, \mathbf{I}} \mathfrak{f} A^{\prime} \approx\left\{s^{*} \mathfrak{f}_{\emptyset, J} \Phi_{u} A^{\prime}\left[\left[-m_{u}\right]\right] ; u \in \mathbf{W}^{J}\right\} .
$$

We replace $s^{*} \mathfrak{e}_{J, \mathbf{I}}$ by $\tilde{e}_{J}$ (see $42.1(\mathrm{f})$ ) and we apply $\tilde{f}_{J}=\mathfrak{f}_{J, \mathbf{I}} s_{!}$(see $42.1(\mathrm{e})$ ); we obtain

$$
\tilde{f}_{J} \tilde{e}_{J} \mathfrak{f} A^{\prime} \approx\left\{\mathfrak{f}_{J, \mathbf{I}} s_{!} s^{*} \mathfrak{f}_{\emptyset, J} \Phi_{u} A^{\prime}\left[\left[-m_{u}\right]\right] ; u \in \mathbf{W}^{J}\right\} .
$$

Using now $42.1(\mathrm{~g})$ we obtain

$$
\tilde{f}_{J} \tilde{e}_{J} \mathfrak{f} A^{\prime} \approx\left\{\mathfrak{f}_{J, \mathbf{I}} \mathfrak{f}_{\emptyset, J} r_{!} r^{*} \Phi_{u} A^{\prime}\left[\left[-m_{u}\right]\right] ; u \in \mathbf{W}^{J}\right\} .
$$

Here we replace $\mathfrak{f}_{J, \mathbf{I}} \mathfrak{f}_{\emptyset, J}$ by $\mathfrak{f}$ (see $36.4(\mathrm{~b})$ ). This (or rather its mixed analogue) gives rise to the following equality in $\mathfrak{K}(D)$ :

$$
\tilde{f}_{J} \tilde{e}_{J} \mathfrak{f}\left(x^{\prime}\right)=\sum_{u \in \mathbf{W}^{J}} v^{2 m_{u}} \mathfrak{f} \rho_{J} \Phi_{u}\left(x^{\prime}\right)
$$


for any $x^{\prime} \in \mathfrak{K}\left(Z_{\emptyset, D}\right)$, or equivalently

$$
f_{J} e_{J} \mathfrak{f}\left(x^{\prime}\right)=\sum_{u \in \mathbf{W}^{J}} v^{2 m_{u}-2 \alpha_{J}} \mathfrak{f} \rho_{J} \Phi_{u}\left(x^{\prime}\right) .
$$

Taking $x^{\prime}=\eta(x)$ where $x \in \mathfrak{K}(C)_{n}^{D}$ (see 41.7) and using 41.7(b) we obtain

$$
\begin{aligned}
& \left(v^{2}-1\right)^{2 \mathbf{r}} f_{J} e_{J} \mathfrak{f} \eta(x) \\
& =\sum_{u \in \mathbf{W}^{J}} \sum_{\lambda \in \underline{\mathfrak{s}}_{n}} v^{-2 l(u)} \mathfrak{f} \rho_{J} \eta\left(\left[u^{-1} ; \lambda\right]^{\prime} * x *\left[\epsilon_{D}(u) ; \underline{D}\left(u^{-1} \lambda\right)\right]^{\prime}\right)
\end{aligned}
$$

and using 42.1(i),

$$
\begin{aligned}
& \left(v^{2}-1\right)^{2 \mathbf{r}} f_{J} e_{J} \mathfrak{f} \eta(x) \\
& =\sum_{u \in \mathbf{W}^{J}} \sum_{\lambda \in \underline{\mathfrak{s}}_{n}} v^{-2 l(u)} \mathfrak{f} \eta \tilde{\rho}_{J}\left(\left[u^{-1} ; \lambda\right]^{\prime} * x *\left[\epsilon_{D}(u) ; \underline{D}\left(u^{-1} \lambda\right)\right]^{\prime}\right)
\end{aligned}
$$

for any $x \in \mathfrak{K}(C)_{n}^{D}$. Here we replace $x$ by $\tilde{\omega}(y)$ where $y \in H_{n, D}$ and $\left.\tilde{\rho}_{J}\right|_{\mathfrak{K}(C)_{n}^{D}}$ by $\left.\tilde{\omega}\right|_{H_{n, D}} \rho_{J, n} \omega_{\mathfrak{K}(C)_{n}^{D}}$; using 40.7(b) we obtain:

$$
\begin{aligned}
& f_{J} e_{J} \mathfrak{f} \eta \tilde{\omega}(y)=\sum_{u \in \mathbf{W}^{J}} \sum_{\lambda \in \underline{\mathfrak{s}}_{n}} \mathfrak{f} \eta \tilde{\omega} \rho_{J, n}\left(\tilde{T}_{u^{-1}} 1_{\lambda} y \tilde{T}_{\epsilon_{D}(u)} 1_{\underline{D}\left(u^{-1} \lambda\right)}\right) \\
& =\mathfrak{f} \eta \tilde{\omega} \rho_{J, n}\left(\sum_{u \in \mathbf{W}^{J}} \tilde{T}_{u^{-1}} y \tilde{T}_{\epsilon_{D}(u)}\right) .
\end{aligned}
$$

The lemma is proved.

42.5. As in 34.12 let $\mathfrak{U}$ be the subfield of $\overline{\mathbf{Q}}_{l}$ generated by the roots of 1 . Let $\Phi: H_{n}^{D} \rightarrow \mathcal{A} \otimes_{\mathbf{z}} H_{n}^{D, \infty}$ be as in 34.12 (a special case of a definition in 34.1) and let $\Phi^{1}: H_{n}^{D, 1} \rightarrow \mathfrak{U} \otimes \mathbf{z} H_{n}^{D, \infty}$ be the specialization of $\Phi$ for $v=1$ (see 34.12(b)). Let $\tilde{\mathcal{A}}=\mathfrak{U}\left[v, v^{-1}\right]$, let $H_{n}^{D, \tilde{\mathcal{A}}}=\tilde{\mathcal{A}} \otimes \mathcal{A} H_{n}^{D}$ and let $\Phi^{\tilde{\mathcal{A}}}: H_{n}^{D, \tilde{\mathcal{A}}} \rightarrow \tilde{\mathcal{A}} \otimes_{\mathbf{z}} H_{n}^{D, \infty}$ be the homomorphism obtained from $\Phi$ by extending the scalars from $\mathcal{A}$ to $\tilde{\mathcal{A}}$.

Let $E$ be an $H_{n}^{D, 1}$-module of finite dimension over $\mathfrak{U}$. Since $\Phi^{1}$ is an isomorphism of $\mathfrak{U}$-algebras (see $34.12(\mathrm{~b})$ ) we may regard $E$ as an $\mathfrak{U} \otimes_{\mathbf{z}} H_{n}^{D, \infty}$-module $E^{\infty}$ via $\left(\Phi^{1}\right)^{-1}$. By extension of scalars, $\tilde{\mathcal{A}} \otimes \mathfrak{U} E^{\infty}$ is naturally a module over

$$
\tilde{\mathcal{A}} \otimes_{\mathfrak{U}}\left(\mathfrak{U} \otimes_{\mathbf{Z}} H_{n}^{D, i y}\right)=\tilde{\mathcal{A}} \otimes_{\mathbf{Z}} H_{n}^{D, i y}
$$

and this can be regarded as an $H_{n}^{D, \tilde{\mathcal{A}}}$-module $E^{\tilde{\mathcal{A}}}$ via $\Phi^{\tilde{\mathcal{A}}}$.

Let $J \subset \mathbf{I}$ be such that $\epsilon(J)=J$. Let $H_{J, n}^{D}$ be the $\mathcal{A}$-algebra of $H_{n}^{D}$ generated by $1_{\lambda}, \lambda \in \underline{\mathfrak{s}}_{n}$ by $\tilde{T}_{w}, w \in \mathbf{W}_{J}$ and by $\tilde{T}_{\underline{D}}$. Note that $\left\{\tilde{T}_{w \underline{D}^{\prime}} 1_{\lambda} ; w \in \mathbf{W}_{J}, \underline{D}^{\prime}=\right.$ power of $\underline{D}\}$ is an $\mathcal{A}$-basis of $H_{J, n}^{D}$. Let $H_{J, n}^{D, 1}=\mathfrak{U} \otimes_{\mathcal{A}} H_{J, n}^{D}$ where $\mathfrak{U}$ is regarded as an $\mathcal{A}$-algebra via $v \mapsto 1$. Let $H_{J, n}^{D, \tilde{\mathcal{A}}}=\tilde{\mathcal{A}} \otimes_{\mathcal{A}} H_{J, n}^{D}$. Note that $H_{J, n}^{D, \tilde{\mathcal{A}}}$ is naturally a subalgebra of $H_{n}^{D, \tilde{\mathcal{A}}}$. Hence $E^{\tilde{\mathcal{A}}}$ may be regarded as an $H_{J, n}^{D, \tilde{\mathcal{A}}}$-module $\left(E^{\tilde{\mathcal{A}}}\right)_{J}$. This $H_{J, n}^{D, \tilde{\mathcal{A}}}$-module may be induced to an $H_{n}^{D, \tilde{\mathcal{A}}}$-module

$$
\operatorname{IND}\left(\left(E^{\tilde{\mathcal{A}}}\right)_{J}\right):=H_{n}^{D, \tilde{\mathcal{A}}} \otimes_{H_{J, n}^{D, \tilde{\mathcal{A}}}} E_{J}^{\tilde{\mathcal{A}}}
$$

Next, $H_{J, n}^{D, 1}$ is naturally a subalgebra of $H_{n}^{D, 1}$. Hence $E$ may be regarded as an $H_{J, n}^{D, 1}$-module $E_{J}$. This $H_{J, n}^{D, 1}$-module may be induced to an $H_{n}^{D, 1}$-module $\operatorname{ind}\left(E_{J}\right):=H_{n}^{D, 1} \otimes_{H_{J, n}^{D, 1}} E_{J}$. Define an $H_{J, n}^{D, \tilde{\mathcal{A}}}$-module $\left(\operatorname{ind}\left(E_{J}\right)\right)^{\tilde{\mathcal{A}}}$ in terms of $\operatorname{ind}\left(E_{J}\right)$ 
in the same way as $E^{\tilde{\mathcal{A}}}$ was defined in terms of $E$. By extension of scalars from $\tilde{\mathcal{A}}$ to $\mathfrak{U}(v)$ (the quotient field of $\tilde{\mathcal{A}}), \operatorname{IND}\left(\left(E^{\tilde{\mathcal{A}}}\right)_{J}\right)$, (ind $\left.\left(E_{J}\right)\right)^{\tilde{\mathcal{A}}}$ give rise to $\mathfrak{U}(v) \otimes_{\mathcal{A}} H_{n}^{D}$ modules $\mathfrak{U}(v) \otimes_{\tilde{\mathcal{A}}} \operatorname{IND}\left(\left(E^{\tilde{\mathcal{A}}}\right)_{J}\right), \mathfrak{U}(v) \otimes_{\tilde{\mathcal{A}}}\left(\operatorname{ind}\left(E_{J}\right)\right)^{\tilde{\mathcal{A}}}$. We show that

(a) these two $\mathfrak{U}(v) \otimes_{\mathcal{A}} H_{n}^{D}$-modules are isomorphic.

Since $\mathfrak{U}(v) \otimes_{\mathcal{A}} H_{n}^{D}, H_{n}^{D, 1}$ are (finite dimensional) semisimple algebras (see 34.12) it follows by standard arguments that it is enough to show that $\operatorname{IND}\left(\left(E^{\tilde{\mathcal{A}}}\right)_{J}\right)$, $\left(\operatorname{ind}\left(E_{J}\right)\right)^{\tilde{\mathcal{A}}}$ become isomorphic $H_{n}^{D, 1}$-modules under the specialization $v=1$. First we note that under the specialization $v=1, E^{\tilde{\mathcal{A}}}$ becomes the $H_{n}^{D, 1}$-module $E$. (This is because the specialization of $\Phi^{\tilde{\mathcal{A}}}$ at $v=1$ cancels $\left(\Phi_{1}\right)^{-1}$.) In particular, the specialization of $\left(\operatorname{ind}\left(E_{J}\right)\right)^{\tilde{\mathcal{A}}}$ for $v=1$ is $\operatorname{ind}\left(E_{J}\right)$. Moreover, from the definition of induction, the specialization of $\operatorname{IND}\left(\left(E^{\tilde{\mathcal{A}}}\right)_{J}\right)$ for $v=1$ is the same as $\operatorname{ind}\left(E_{J}^{\prime}\right)$ where $E^{\prime}$ is the specialization of $E^{\tilde{\mathcal{A}}}$ for $v=1$, that is, $E^{\prime}=E$. This proves (a).

Lemma 42.6. We preserve the setup of 42.5. Let $\mathfrak{U}(v) \otimes_{\tilde{\mathcal{A}}} E^{\tilde{\mathcal{A}}}, \mathfrak{U}(v) \otimes_{\tilde{\mathcal{A}}}\left(\operatorname{ind}\left(E_{J}\right)\right)^{\tilde{\mathcal{A}}}$ be the $\mathfrak{U}(v) \otimes_{\mathcal{A}} H_{n}^{D}$-module obtained from $E^{\tilde{\mathcal{A}}}$, $\left(\operatorname{ind}\left(E_{J}\right)\right)^{\tilde{\mathcal{A}}}$ by extension of scalars from $\tilde{\mathcal{A}}$ to $\mathfrak{U}(v)$. Let $y \in H_{n, D}$. We have

$$
\operatorname{tr}\left(\delta_{J}(y) \tilde{T}_{\underline{D}}, \mathfrak{U}(v) \otimes_{\tilde{\mathcal{A}}} E^{\tilde{\mathcal{A}}}\right)=\operatorname{tr}\left(y \tilde{T}_{\underline{D}}, \mathfrak{U}(v) \otimes_{\tilde{\mathcal{A}}}\left(\operatorname{ind}\left(E_{J}\right)\right)^{\tilde{\mathcal{A}}}\right) .
$$

Let $H_{J, n}$ be the $\mathcal{A}$-subalgebra of $H_{n}$ defined in 31.8. Define an $\mathcal{A}$-linear map $p_{J}: H_{n} \rightarrow H_{J, n}$ by $\left.p_{J}\left(\tilde{T}_{z} 1_{\lambda}\right)\right)=\tilde{T}_{z} 1_{\lambda}$ if $z \in \mathbf{W}_{J}, \lambda \in \underline{\mathfrak{s}}_{n}, p_{J}\left(\tilde{T}_{z} 1_{\lambda}\right)=0$ if $z \in$ $\mathbf{W}-\mathbf{W}_{J}, \lambda \in \underline{\mathfrak{s}}_{n}$. We show that

(a) $p_{J}\left(\tilde{T}_{u} h^{\prime}\right)=\delta_{u, 1} h^{\prime}$ if $u \in \mathbf{W}^{J}, h^{\prime} \in H_{J, n}$.

We may assume that $h^{\prime}=\tilde{T}_{b} 1_{\lambda}, b \in \mathbf{W}_{J}, \lambda \in \underline{\mathfrak{s}}_{n}$. Then $p_{J}\left(\tilde{T}_{u} \tilde{T}_{b} 1_{\lambda}\right)=p_{J}\left(\tilde{T}_{u b} 1_{\lambda}\right)=$ $\delta_{u, 1} \tilde{T}_{u b} 1_{\lambda}=\delta_{u, 1} \tilde{T}_{b} 1_{\lambda}$, as required.

We show that

(b) $p_{J}\left(h h^{\prime}\right)=p_{J}(h) h^{\prime}$ for any $h \in H_{n}, h^{\prime} \in H_{J, n}$.

We may assume $h=\tilde{T}_{u} \tilde{T}_{b} 1_{\nu}, h^{\prime}=\tilde{T}_{a} 1_{\lambda}, u \in \mathbf{W}^{J}, a, b \in \mathbf{W}_{J}, \lambda, \nu \in \underline{\mathfrak{s}}_{n}$. We must show that $p_{J}\left(\tilde{T}_{u} \tilde{T}_{b} 1_{\nu} \tilde{T}_{a} 1_{\lambda}\right)=p_{J}\left(\tilde{T}_{u} \tilde{T}_{b} 1_{\nu}\right) \tilde{T}_{a} 1_{\lambda}$. If $u \neq 1$, both sides are zero by (a). If $u=1$, both sides are $\tilde{T}_{b} 1_{\nu} \tilde{T}_{a} 1_{\lambda}$. This proves (b).

By 34.13(a) we have

(c) $p_{\emptyset}\left(\tilde{T}_{x} \tilde{T}_{x^{\prime}} 1_{\lambda}\right)=\delta_{x x^{\prime}, 1}$ for $x, x^{\prime} \in \mathbf{W}, \lambda \in \underline{\mathfrak{s}}_{n}$.

For $u, u^{\prime} \in \mathbf{W}^{J}, \lambda \in \underline{\mathfrak{g}}_{n}$ we write $\tilde{T}_{u^{-1}} \tilde{T}_{u^{\prime}} 1_{\lambda}=\sum_{a \in \mathbf{W}} f_{a} \tilde{T}_{a} 1_{\lambda}$ where $f_{a} \in \mathcal{A}$. For $a^{\prime} \in \mathbf{W}_{J}$ we have

$$
\tilde{T}_{a^{\prime-1} u^{-1}} \tilde{T}_{u^{\prime}} 1_{\lambda}=\tilde{T}_{a^{\prime-1}} \tilde{T}_{u^{-1}} \tilde{T}_{u^{\prime}} 1_{\lambda}=\sum_{a \in \mathbf{W}} f_{a} \tilde{T}_{a^{\prime-1}} \tilde{T}_{a} 1_{\lambda} .
$$

Applying $p_{\emptyset}$ to this and using (c) gives $f_{a^{\prime}}=\delta_{u^{\prime}, u a^{\prime}}=\delta_{a^{\prime}, 1} \delta_{u, u^{\prime}}$ so that

$$
p_{J}\left(\tilde{T}_{u^{-1}} \tilde{T}_{u^{\prime}} 1_{\lambda}\right)=\sum_{a \in \mathbf{W}_{J}} f_{a} \tilde{T}_{a} 1_{\lambda}=\delta_{u, u^{\prime}} \tilde{T}_{1} 1_{\lambda} .
$$

Since this holds for any $\lambda \in \underline{\mathfrak{s}}_{n}$ we have

(d) $p_{J}\left(\tilde{T}_{u^{-1}} \tilde{T}_{u^{\prime}}\right)=\delta_{u, u^{\prime}} \tilde{T}_{1}$.

Let $w \in \mathbf{W}, \lambda \in \underline{\mathfrak{s}}_{n}, u \in \mathbf{W}^{J}$. We have

$$
\tilde{T}_{w} 1_{\lambda} \tilde{T}_{u}=\sum_{u^{\prime} \in \mathbf{W}^{J}, a \in \mathbf{W}_{J}} c_{w, u, u^{\prime}, a, \lambda} \tilde{T}_{u^{\prime}} \tilde{T}_{a} 1_{u^{-1} \lambda}
$$


where $c_{w, u, u^{\prime}, a, \lambda} \in \mathcal{A}$ are uniquely determined. It follows that

$$
\tilde{T}_{u^{-1}} \tilde{T}_{w} 1_{\lambda} \tilde{T}_{\epsilon(u)}=\sum_{u^{\prime} \in \mathbf{W}^{J}, a \in \mathbf{W}_{J}} c_{w, \epsilon(u), u^{\prime}, a, \lambda} \tilde{T}_{u^{-1}} \tilde{T}_{u^{\prime}} \tilde{T}_{a} 1_{\epsilon(u)^{-1} \lambda} .
$$

Applying $p_{J}$ and using (b),(d) we obtain

$$
\begin{gathered}
p_{J}\left(\tilde{T}_{u^{-1}} \tilde{T}_{w} 1_{\lambda} \tilde{T}_{\epsilon(u)}\right)=\sum_{u^{\prime} \in \mathbf{W}^{J}, a \in \mathbf{W}_{J}} c_{w, \epsilon(u), u^{\prime}, a, \lambda} p_{J}\left(\tilde{T}_{u^{-1}} \tilde{T}_{u^{\prime}}\right) \tilde{T}_{a} 1_{\epsilon(u)^{-1} \lambda} \\
(\mathrm{e})=\sum_{u^{\prime} \in \mathbf{W}^{J}, a \in \mathbf{W}_{J}} c_{w, \epsilon(u), u^{\prime}, a, \lambda} \delta_{u, u^{\prime}} \tilde{T}_{a} 1_{\epsilon(u)^{-1} \lambda}=\sum_{a \in \mathbf{W}_{J}} c_{w, \epsilon(u), u, a, \lambda} \tilde{T}_{a} 1_{\epsilon(u)^{-1} \lambda .} .
\end{gathered}
$$

Let $\left(e_{i}\right)_{i \in X}$ be a basis of the free $\tilde{\mathcal{A}}$-module $E^{\tilde{\mathcal{A}}}$. For $a \in \mathbf{W}_{J}, \lambda \in \underline{\mathfrak{s}}_{n}$ we have $\tilde{T}_{a} 1_{\lambda} \tilde{T}_{D} e_{i}=\sum_{i^{\prime} \in X} \tilde{c}_{a, \lambda, i, i^{\prime}} e_{i^{\prime}}$ where $\tilde{c}_{a, \lambda, i, i^{\prime}} \in \tilde{\mathcal{A}}$.

Since $H_{n}^{D, \tilde{\mathcal{A}}}$ is a free right $H_{J, n}^{D, \tilde{\mathcal{A}}}$-module with basis $\left\{\tilde{T}_{u} ; u \in \mathbf{W}^{J}\right\}$, we see that $\left\{\tilde{T}_{u} \otimes e_{i} ; u \in \mathbf{W}^{J}, i \in X\right\}$ is a basis of the free $\tilde{\mathcal{A}}$-module $\operatorname{ind}\left(\left(E^{\tilde{\mathcal{A}}}\right)_{J}\right)$.

Let $w \in \mathbf{W}, \lambda \in \underline{\mathfrak{s}}_{n}, u \in \mathbf{W}^{J}$ be such that $w \underline{D} \lambda=\lambda$. In $\operatorname{IND}\left(\left(E^{\tilde{\mathcal{A}}}\right)_{J}\right)$ we have

$$
\begin{aligned}
& \tilde{T}_{w} 1_{\lambda} \tilde{T}_{\underline{D}}\left(\tilde{T}_{u} \otimes e_{i}\right)=\left(\tilde{T}_{w} 1_{\lambda} \tilde{T}_{\epsilon(u)} \tilde{T}_{\underline{D}}\right) \otimes e_{i} \\
& =\sum_{u^{\prime} \in \mathbf{W}^{J}, a \in \mathbf{W}_{J}} c_{w, \epsilon(u), u^{\prime}, a, \lambda}\left(\tilde{T}_{u^{\prime}} \tilde{T}_{a} 1_{\epsilon(u)^{-1} \lambda} \tilde{T}_{\underline{D}}\right) \otimes e_{i} \\
& =\sum_{u^{\prime} \in \mathbf{W}^{J}, a \in \mathbf{W}_{J}} c_{w, \epsilon(u), u^{\prime}, a, \lambda} \tilde{T}_{u^{\prime}} \otimes\left(\tilde{T}_{a} 1_{\epsilon(u)^{-1} \lambda} \tilde{T}_{D} e_{i}\right) \\
& =\sum_{u^{\prime} \in \mathbf{W}^{J}, a \in \mathbf{W}_{J}, i^{\prime} \in X} c_{w, \epsilon(u), u^{\prime}, a, \lambda} \tilde{c}_{a, \epsilon(u)^{-1} \lambda, i, i^{\prime}} \tilde{T}_{u^{\prime}} \otimes e_{i^{\prime}} .
\end{aligned}
$$

Hence, using (e),

$$
\begin{aligned}
& \operatorname{tr}\left(\tilde{T}_{w} 1_{\lambda} \tilde{T}_{\underline{D}}, \operatorname{IND}\left(\left(E^{\tilde{\mathcal{A}}}\right)_{J}\right)\right)=\sum_{u \in \mathbf{W}^{J}, a \in \mathbf{W}_{J}, i \in X} c_{w, \epsilon(u), u, a, \lambda} \tilde{c}_{a, \epsilon(u)^{-1} \lambda, i, i} \\
& =\sum_{u \in \mathbf{W}^{J}, a \in \mathbf{W}_{J}} c_{w, \epsilon(u), u, a, \lambda} \operatorname{tr}\left(\tilde{T}_{a} 1_{\epsilon(u)^{-1} \lambda} \tilde{T}_{\underline{D}}, E^{\tilde{\mathcal{A}}}\right) \\
& =\sum_{u \in \mathbf{W}^{J}} \operatorname{tr}\left(\sum_{a \in \mathbf{W}_{J}} c_{w, \epsilon(u), u, a, \lambda} \tilde{T}_{a} 1_{\epsilon(u)^{-1} \lambda} \tilde{T}_{\underline{D}}, E^{\tilde{\mathcal{A}}}\right) \\
& =\operatorname{tr}\left(p_{J}\left(\sum_{u \in \mathbf{W}^{J}} \tilde{T}_{u^{-1}} \tilde{T}_{w} 1_{\lambda} \tilde{T}_{\epsilon(u)}\right) \tilde{T}_{\underline{D}}, E^{\tilde{\mathcal{A}}}\right) \\
& =\operatorname{tr}\left(\rho_{J, n}\left(\sum_{u \in \mathbf{W}^{J}} \tilde{T}_{u^{-1}} \tilde{T}_{w} 1_{\lambda} \tilde{T}_{\epsilon(u)}\right) \tilde{T}_{\underline{D}}, E^{\tilde{\mathcal{A}}}\right)=\operatorname{tr}\left(\delta_{J}\left(\tilde{T}_{w} 1_{\lambda}\right) \tilde{T}_{\underline{D}}, E^{\tilde{\mathcal{A}}}\right) .
\end{aligned}
$$

Thus we have

$$
\operatorname{tr}\left(\delta_{J}\left(\tilde{T}_{w} 1_{\lambda}\right) \tilde{T}_{\underline{D}}, E^{\tilde{\mathcal{A}}}\right)=\operatorname{tr}\left(\tilde{T}_{w} 1_{\lambda} \tilde{T}_{\underline{D}}, \operatorname{IND}\left(\left(E^{\tilde{\mathcal{A}}}\right)_{J}\right)\right)=\operatorname{tr}\left(\tilde{T}_{w} 1_{\lambda} \tilde{T}_{\underline{D}},\left(\operatorname{ind}\left(E_{J}\right)\right)^{\tilde{\mathcal{A}}}\right)
$$

where the second equality follows from 42.5 (a). Since the elements $\tilde{T}_{w} 1_{\lambda}$ as above generate the $\mathcal{A}$-module $H_{n, D}$, the lemma follows.

42.7. Let $\mathcal{V}$ be the $\mathbf{Q}$-vector subspace of $\mathbf{Q} \otimes \operatorname{Hom}\left(\mathbf{k}^{*}, \mathbf{T}\right)$ spanned by the coroots. Let $\mathcal{V}_{\mathbf{R}}=\mathbf{R} \otimes_{\mathbf{Q}} \mathcal{V}$. The kernels of the roots $\mathcal{V}_{\mathbf{R}} \rightarrow \mathbf{R}$ a hyperplane arrangement which defines a partition of $\mathcal{V}_{\mathbf{R}}$ into facets in a standard way. Let $\mathcal{F}$ be the set of facets. Now the orbits of $\mathbf{W}$ on $\mathcal{F}$ are naturally indexed by the various subsets $J$ 
of I. This gives a partition $\mathcal{F}=\bigsqcup_{J \subset \mathbf{I}} \mathcal{F}_{J}$. For example, $\mathcal{F}_{\emptyset}$ consists of all Weyl chambers. If $F \in \mathcal{F}_{J}$, then $F$ is homeomorphic to a real affine space of dimension $|\mathbf{I}-J|$ hence we have $H_{c}^{i}(F)=0$ if $i \neq|\mathbf{I}-J|$ and $H_{c}^{|\mathbf{I}-J|}(F)=\Lambda^{|\mathbf{I}-J|}[F]$; here we write $H_{c}^{i}(?)$ instead of $H_{c}^{i}(?, \mathbf{R}),[F]$ denotes the vector subspace of $\mathcal{V}_{\mathbf{R}}$ in which $F$ is open dense and $\Lambda^{|\mathbf{I}-J|}[F]$ is the top exterior power of $[F]$. Note that $[F]=\mathbf{R} \otimes_{\mathbf{Q}}\left([F]_{\mathbf{Q}}\right)$ for a well-defined $\mathbf{Q}$-subspace $[F]_{\mathbf{Q}}$ of $\mathcal{V}$. For any $\underline{D}$-orbit $\eta$ on the set of subsets of $\mathbf{I}$ let $\mathcal{V}_{\mathbf{R}}^{\eta}=\bigcup_{J \in \eta} \bigcup_{F \in \mathcal{F}_{J}} F \subset \mathcal{V}_{\mathbf{R}}$ and let $r_{\eta}=|\mathbf{I}-J|$ for some/any $J \in \eta$. We have $H_{c}^{i}\left(\mathcal{V}_{\mathbf{R}}^{\eta}\right)=0$ if $i \neq r_{\eta}, H_{c}^{r_{\eta}}\left(\mathcal{V}_{\mathbf{R}}^{\eta}\right)=\bigoplus_{J \in \eta} \bigoplus_{F \in \mathcal{F}_{J}} \Lambda^{r_{\eta}}[F]$. Note also that $H_{c}^{i}\left(\mathcal{V}_{\mathbf{R}}\right)=0$ if $i \neq|\mathbf{I}|$ and $H_{c}^{|\mathbf{I}|}\left(\mathcal{V}_{\mathbf{R}}\right)=\Lambda^{|\mathbf{I}|} \mathcal{V}_{\mathbf{R}}$. The $\mathbf{W}^{D}$-action on $\mathbf{T}$ induces a linear action of $\mathbf{W}^{D}$ on $\mathcal{V}_{\mathbf{R}}$. This action restricts for any $\eta$ to a $\mathbf{W}^{D}$ action on $\mathcal{V}_{\mathbf{R}}^{\eta}$ and this induces a $\mathbf{W}^{D}$-action on $H_{c}^{r_{\eta}}\left(\mathcal{V}_{\mathbf{R}}^{\eta}\right)$. It also induces a natural $\mathbf{W}^{D}$-action on $H_{c}^{|\mathbf{I}|}\left(\mathcal{V}_{\mathbf{R}}\right)=\Lambda^{|\mathbf{I}|} \mathcal{V}_{\mathbf{R}}$. The long cohomology exact sequences attached to the partition $\mathcal{V}_{\mathbf{R}}=\bigcup_{\eta} \mathcal{V}_{\mathbf{R}}^{\eta}$ show that $(-1)^{|\mathbf{I}|} H_{c}^{|\mathbf{I}|}\left(\mathcal{V}_{\mathbf{R}}\right)=\sum_{\eta}(-1)^{r_{\eta}} H_{c}^{r_{\eta}}\left(\mathcal{V}_{\mathbf{R}}^{\eta}\right)$ in the Grothendieck group of representations of $\mathbf{W}^{D}$ over $\mathbf{R}$, that is,

$$
\begin{aligned}
& \Lambda^{|\mathbf{I}|} \mathcal{V}_{\mathbf{R}} \oplus \bigoplus_{\eta ; r_{\eta}=|\mathbf{I}|+1} \bigoplus_{\bmod 2}\left(\bigoplus_{J \in \eta} \bigoplus_{F \in \mathcal{F}_{J}} \Lambda^{r_{\eta}}[F]\right) \\
& \cong \bigoplus_{\eta ; r_{\eta}=|\mathbf{I}|}\left(\bigoplus_{\bmod 2} \bigoplus_{J \in \eta} \Lambda_{F \in \mathcal{F}_{J}}^{r_{\eta}}[F]\right)
\end{aligned}
$$

as representations of $\mathbf{W}^{D}$ over $\mathbf{R}$. All real representations in this formula come naturally from representations of $\mathbf{W}^{D}$ over $\mathbf{Q}$. Hence the previous formula remains valid (as representations of $\mathbf{W}^{D}$ over $\mathbf{Q}$ ) if $\mathcal{V}_{\mathbf{R}},[F]$ are replaced by $\mathcal{V},[F]_{\mathbf{Q}}$ and the exterior powers are taken over $\mathbf{Q}$. Tensoring both sides (over $\mathbf{Q}$ ) by $\mathfrak{U}$ (as in 42.5 ) we obtain

$$
\begin{aligned}
& \Lambda^{|\mathbf{I}|} \mathcal{V}_{\mathfrak{U}} \oplus \bigoplus_{\eta ; r_{\eta}=|\mathbf{I}|+1} \bigoplus_{\eta \bmod 2}\left(\bigoplus_{J \in \eta} \bigoplus_{F \in \mathcal{F}_{J}} \Lambda^{r_{\eta}}[F]_{\mathfrak{U}}\right) \\
& \cong \bigoplus_{\eta ; r_{\eta}=|\mathbf{I}|}\left(\bigoplus_{\bmod 2} \bigoplus_{J \in \eta} \Lambda_{F \in \mathcal{F}_{J}}^{r_{\eta}}[F]_{\mathfrak{U}}\right)
\end{aligned}
$$

as representations of $\mathbf{W}^{D}$ over $\mathfrak{U}$; here $\mathcal{V}_{\mathfrak{U}}=\mathfrak{U} \otimes_{\mathbf{Q}} \mathcal{V},[F]_{\mathfrak{U}}=\mathfrak{U} \otimes_{\mathbf{Q}}[F]_{\mathbf{Q}}$ and the exterior powers are taken over $\mathfrak{U}$. We may view (a) as an isomorphism of $H_{n}^{D, 1_{-}}$ modules: the $\mathbf{W}^{D}$-modules in (a) may be viewed as $H_{n}^{D, 1}$-modules via the algebra homomorphism $H_{n}^{D, 1} \rightarrow \mathfrak{U}\left[\mathbf{W}^{D}\right]$ given by $\tilde{T}_{w} \mapsto w$ for $w \in \mathbf{W}^{D}, 1_{\lambda} \mapsto 0$ for $\lambda \neq \lambda_{0}$, $1_{\lambda_{0}} \mapsto 1$ (here $\lambda_{0}$ is the neutral element of the abelian group $\underline{\mathfrak{s}}_{n}$; see 28.1).

We define an $\mathfrak{U}$-linear map $\Delta: H_{n}^{D, 1} \rightarrow H_{n}^{D, 1} \otimes H_{n}^{D, 1}$ by $\Delta\left(\tilde{T}_{w}\right)=\tilde{T}_{w} \otimes \tilde{T}_{w}$ for $w \in \mathbf{W}^{D}$ and $\Delta\left(1_{\lambda}\right)=\sum_{\lambda_{1}, \lambda_{2} \in \underline{\mathfrak{s}}_{n} ; \lambda_{1} \lambda_{2}=\lambda} 1_{\lambda_{1}} \otimes 1_{\lambda_{2}}$ for $\lambda \in \underline{\mathfrak{s}}_{n}$. (Here we use the abelian group structure on $\underline{\mathfrak{s}}_{n}$, a subgroup of $\underline{\mathfrak{s}}$; see 28.1.) This makes $H_{n}^{D, 1}$ into a Hopf algebra. (Note that the analogous formulas do not make $H_{n}^{D}$ into a Hopf algebra.) It follows that for any two $H_{n}^{D, 1}$-modules $E_{1}, E_{2}$, the $\mathfrak{U}$-vector space $E_{1} \otimes E_{2}$ is naturally an $H_{n}^{D, 1}$-module.

Now let $E$ be an $H_{n}^{D, 1}$-module of finite dimension over $\mathfrak{U}$. Then we can take the tensor product of each $H_{n}^{D, 1}$-module in (a) with $E$ and we obtain an isomorphism of $H_{n}^{D, 1}$-modules

$$
E \otimes \Lambda^{|\mathbf{I}|} \mathcal{V}_{\mathfrak{U}} \oplus \bigoplus_{\eta ; r_{\eta}=|\mathbf{I}|+1} X_{\bmod 2} \cong \bigoplus_{\eta ; r_{\eta}=|\mathbf{I}| \bmod 2} X_{\eta}
$$


where $X_{\eta}=E \otimes \bigoplus_{J \in \eta} \bigoplus_{F \in \mathcal{F}_{J}} \Lambda^{r_{\eta}}[F]_{\mathfrak{U}}$. Applying to this the functor $E \mapsto E^{\tilde{\mathcal{A}}}$ (see 42.5), we deduce an isomorphism of $H_{n}^{D, \tilde{\mathcal{A}} \text {-modules }}$

$$
\left(E \otimes \Lambda^{|\mathbf{I}|} \mathcal{V}_{\mathfrak{U}}\right)^{\tilde{\mathcal{A}}} \oplus \bigoplus_{\eta ; r_{\eta}=|\mathbf{I}|+1} X_{\bmod 2}^{\tilde{\mathcal{A}}} \cong \bigoplus_{\eta ; r_{\eta}=|\mathbf{I}|} X_{\bmod 2}^{\tilde{\mathcal{A}}} .
$$

We deduce that for $y \in H_{n, D}$ we have

$$
\operatorname{tr}\left(y \tilde{T}_{\underline{D}},\left(E \otimes \Lambda^{|\mathbf{I}|} \mathcal{V}_{\mathfrak{U}}\right)^{\tilde{\mathcal{A}}}\right)=\sum_{\eta}(-1)^{r_{\eta}+|\mathbf{I}|} \operatorname{tr}\left(y \tilde{T}_{\underline{D}}, X_{\eta}^{\tilde{\mathcal{A}}}\right) .
$$

We have $X_{\eta}=\bigoplus_{J \in \eta} X^{J}$ where $X^{J}=E \otimes\left(\bigoplus_{F \in \mathcal{F}_{J}} \Lambda^{r_{\eta}}[F]_{\mathfrak{L}}\right)$.

Assume first that $\eta$ consists of at least two subsets of $\mathbf{I}$. Then each $X_{J}$ is stable under $H_{n}^{D, 1}$ and is mapped by $\tilde{T}_{\underline{D}}$ into $X_{J^{\prime}}$ with $J \neq J^{\prime}$. From the definitions we have $X_{\eta}^{\tilde{\mathcal{A}}}=\bigoplus_{J \in \eta} \tilde{\mathcal{A}} \otimes_{\mathfrak{U}} X_{J}$ as an $\tilde{\mathcal{A}}$-module and each summand $\tilde{\mathcal{A}} \otimes_{\mathfrak{U}} X_{J}$ is stable under $H_{n}$ and is mapped by $\tilde{T}_{\underline{D}}$ into a summand $\tilde{\mathcal{A}} \otimes_{\mathfrak{U}} X_{J^{\prime}}$ with $J \neq J^{\prime}$. It follows that for our $\eta$ we have

$$
\text { (c) } \operatorname{tr}\left(y \tilde{T}_{\underline{D}}, X_{\eta}^{\tilde{\mathcal{A}}}\right)=0 \text {. }
$$

Next assume that $\eta$ consists of a single subset $J$ of $\mathbf{I}$. We have $\underline{D}(J)=J$. Let $F_{J}$ be the unique facet in $\mathcal{F}_{J}$ such that $F_{J}$ is contained in the closure of the dominant Weyl chamber. Then $F_{J}$ is stable under the the subgroup $\mathbf{W}_{J}^{D}$ of $\mathbf{W}^{D}$ generated by $\mathbf{W}_{J}$ and $\underline{D}$ and $X_{\eta}$ may be identified with $E \otimes\left(H_{n}^{D, 1} \otimes_{H_{J, n}^{D, 1}}\left(\Lambda^{|\mathbf{I}-J|}\left[F_{J}\right]_{\mathfrak{U}}\right)\right)$. Here $\Lambda^{|\mathbf{I}-J|}\left[F_{J}\right]_{\mathfrak{U}}$ is regarded as a $W W_{J}^{D}$-module and then is viewed as a $H_{J, n}^{D, 1}$ module via the canonical algebra homomorphism $H_{J, n}^{D, 1} \rightarrow \mathfrak{U}\left[\mathbf{W}_{J}^{D}\right]$; thus $1_{\lambda}$ acts on it as 1 if $\lambda=\lambda_{0}$ and as 0 if $\lambda \neq \lambda_{0}$. Note that in the $\mathbf{W}_{J}^{D}$-module $\Lambda^{|\mathbf{I}-J|}\left[F_{J}\right]_{\mathfrak{U}}$, $\mathbf{W}_{J}$ acts trivially (since $\mathbf{W}_{J}$ acts trivially on $\left[F_{J}\right]_{\mathfrak{U}}$ ) and $\underline{D}$ acts as multiplication by $\left.(-1)^{|\mathbf{I}-J|-\left|(\mathbf{I}-J)_{\epsilon}\right|}\right)$. Let $X_{\eta}^{\prime}=E \otimes\left(H_{n}^{D, 1} \otimes_{H_{J, n}^{D, 1}} \mathfrak{U}\right)$ where $\mathfrak{U}$ is regarded as a $H_{J, n}^{D, 1}$-module coming from the trivial representation of $\mathbf{W}_{J}^{D}$. We see that we may identify $X_{\eta}, X_{\eta}^{\prime}$ in a way compatible with the $H_{n}^{1}$-module structures and so that the action of $\tilde{T}_{\underline{D}}$ on $X_{\eta}$ corresponds to $(-1)^{|\mathbf{I}-J|-\left|(\mathbf{I}-J)_{\epsilon}\right|}$ times the action of $\tilde{T}_{\underline{D}}$ on $X_{\eta}^{\prime}$. Using the definitions we see that we may identify $X_{\eta}^{\tilde{\mathcal{A}}}, X_{\eta}^{\prime} \tilde{\mathcal{A}}$ in a way compatible with the $H_{n}$-module structures and so that the action of $\tilde{T}_{\underline{D}}$ on $X_{\eta}^{\tilde{\mathcal{A}}}$ corresponds to $(-1)^{|\mathbf{I}-J|-\left|(\mathbf{I}-J)_{\epsilon}\right|}$ times the action of $\tilde{T}_{\underline{D}}$ on $X_{\eta}^{\prime} \tilde{\mathcal{A}}$. From the definitions we have $X_{\eta}^{\prime}=\operatorname{ind}\left(E_{J}\right)$ (notation of 42.5). We see that for our $\eta$ we have

$$
\operatorname{tr}\left(y \tilde{T}_{\underline{D}}, X_{\eta}^{\tilde{\mathcal{A}}}\right)=(-1)^{|\mathbf{I}-J|-\left|(\mathbf{I}-J)_{\epsilon}\right|} \operatorname{tr}\left(y \tilde{T}_{\underline{D}},\left(\operatorname{ind}\left(E_{J}\right)\right)^{\tilde{\mathcal{A}}}\right) .
$$

From the definitions (34.4) we see that there is a unique $\tilde{\mathcal{A}}$-algebra homomorphism $\vartheta: H_{n}^{D, \tilde{\mathcal{A}}} D \rightarrow H_{n}^{D, \tilde{\mathcal{A}}}$ such that

$\vartheta\left(1_{\lambda}\right)=1_{\lambda}$ for any $\lambda \in \underline{\mathfrak{s}}_{n}$,

$\vartheta\left(\tilde{T}_{w}\right)=(-1)^{l(w)} \tilde{T}_{w^{-1}}^{-1}$ for any $w \in \mathbf{W}$,

$\vartheta\left(\tilde{T}_{\underline{D}}\right)=(-1)^{|\mathbf{I}|-\left|\mathbf{I}_{\epsilon}\right|} \tilde{T}_{\underline{D}}$.

We have $\vartheta^{2}=1$.

Using $\vartheta$ and $E^{\tilde{\mathcal{A}}}$ we can define a new $H_{n}^{D, \tilde{\mathcal{A}}}$-module $E^{\tilde{\mathcal{A}}, \vartheta}$ with the same underlying $\tilde{\mathcal{A}}$-module as $E^{\tilde{\mathcal{A}}}$ but with $x \in H_{n}^{D, \tilde{\mathcal{A}}}$ acting on $E^{\tilde{\mathcal{A}}, \vartheta}$ in the same way that $\vartheta(x)$ acts on $E^{\tilde{\mathcal{A}}}$. We show that 
(e) under extension of scalars from $\tilde{\mathcal{A}}$ to $\mathfrak{U}(v)$, the $H_{n}^{D, \tilde{\mathcal{A}}}$-modules $E^{\tilde{\mathcal{A}}, \vartheta}$ and $\left(E \otimes \Lambda^{|\mathbf{I}|} \mathcal{V}_{\mathfrak{U}}\right)^{\tilde{\mathcal{A}}}$ become isomorphic $\mathfrak{U}(v) \otimes_{\mathcal{A}} H_{n}^{D}$-modules.

As in the proof of 42.5 (a) it is enough to show that these $H_{n}^{D, \tilde{\mathcal{A}}}$-modules become isomorphic $H_{n}^{D, 1}$-modules under the specialization $v=1$. Thus it is enough to show that $\left.E^{\tilde{\mathcal{A}}, \vartheta}\right|_{v=1} \cong E \otimes \Lambda^{|\mathbf{I}|} \mathcal{V}_{\mathfrak{U}}$ as $H_{n}^{D, 1}$-modules. Now the underlying $\mathfrak{U}$-vector space of $\left.E^{\tilde{\mathcal{A}}, \vartheta}\right|_{v=1}$ is $E$ but the action of $x \in H_{n}^{D, 1}$ on $\left.E^{\tilde{\mathcal{A}}, \vartheta}\right|_{v=1}$ is the same as the action of $\vartheta_{1}(x)$ on $E$. Here $\vartheta_{1}: H_{n}^{D, 1} \rightarrow H_{n}^{D, 1}$ is the specialization of $\vartheta_{1}$ for $v=1$. Note that $\vartheta_{1}\left(1_{\lambda}\right)=1_{\lambda}$ for any $\lambda \in \underline{\mathfrak{s}}_{n}$ and $\vartheta_{1}\left(\tilde{T}_{w}\right)=\gamma_{w} \tilde{T}_{w}$ for any $w \in \mathbf{W}^{D}$, where $\gamma_{w}= \pm 1$ is the scalar by which $w$ acts in the $\mathbf{W}^{D}$-module $\Lambda^{|\mathbf{I}|} \mathcal{V}_{\mathfrak{U}}$. The desired result follows.

Combining (b),(c),(d),(e) we see that for any $y \in H_{n, D}$ we have

$$
(-1)^{|\mathbf{I}|+\left|\mathbf{I}_{\epsilon}\right|} \operatorname{tr}\left(\vartheta\left(y \tilde{T}_{\underline{D}}\right), E^{\tilde{\mathcal{A}}}\right)=\sum_{J \subset \mathbf{I} ; \epsilon(J)=J}(-1)^{\left|J_{\epsilon}\right|} \operatorname{tr}\left(y \tilde{T}_{\underline{D}},\left(\operatorname{ind}\left(E_{J}\right)\right)^{\tilde{\mathcal{A}}}\right) .
$$

Replacing here $(-1)^{|\mathbf{I}|+\left|\mathbf{I}_{\epsilon}\right|} \vartheta\left(y \tilde{T}_{\underline{D}}\right)$ by $\vartheta(y) \tilde{T}_{\underline{D}}$ and using Lemma 42.6 we may rewrite this as

$$
\operatorname{tr}\left(\vartheta(y) \tilde{T}_{\underline{D}}, E^{\tilde{\mathcal{A}}}\right)=\sum_{J \subset \mathbf{I} ; \epsilon(J)=J}(-1)^{\left|J_{\epsilon}\right|} \operatorname{tr}\left(\delta_{J}(y) \tilde{T}_{\underline{D}}, E^{\tilde{\mathcal{A}}}\right)
$$

or equivalently (see 42.4) $\operatorname{tr}\left(\vartheta(y) \tilde{T}_{\underline{D}}, E^{\tilde{\mathcal{A}}}\right)=\operatorname{tr}\left(\delta(y) \tilde{T}_{\underline{D}}, E^{\tilde{\mathcal{A}}}\right)$. Since any simple $\mathfrak{U}(v) \otimes_{\mathcal{A}} H_{n}^{D}$-module can be obtained by extension of scalars (from $\tilde{\mathcal{A}}$ to $\mathfrak{U}(v)$ ) from some $E^{\tilde{\mathcal{A}}}$ as above, we deduce that

$$
\operatorname{tr}\left((\delta(y)-\vartheta(y)) \tilde{T}_{\underline{D}}, \mathbf{E}\right)=0
$$

for any simple $\mathfrak{U}(v) \otimes_{\mathcal{A}} H_{n}^{D}$-module E. Since $\mathfrak{U}(v) \otimes_{\mathcal{A}} H_{n}^{D}$ is a semisimple algebra, it follows that $(\delta(y)-\vartheta(y)) \tilde{T}_{\underline{D}}$ belongs to the $\mathfrak{U}(v)$-subspace of $\mathfrak{U}(v) \otimes_{\mathcal{A}} H_{n}^{D}$ spanned by commutators $x x^{\prime}-x^{\prime} x$ with $x, x^{\prime} \in \mathfrak{U}(v) \otimes_{\mathcal{A}} H_{n}^{D}$. Hence we have

$$
g(\delta(y)-\vartheta(y)) \tilde{T}_{\underline{D}}=\sum_{i=1}^{m} g_{i}\left(x_{i} \tilde{T}_{\underline{D}}^{s_{i}} x_{i}^{\prime} \tilde{T}_{\underline{D}}^{1-s_{i}}-x_{i}^{\prime} \tilde{T}_{\underline{D}}^{1-s_{i}} x_{i} \tilde{T}_{\underline{D}}^{s_{i}}\right)
$$

with $g \in \mathcal{A}-\{0\}, g_{i} \in \mathcal{A}, x_{i} \in H_{n}, x_{i}^{\prime} \in H_{n}, s_{i} \in \mathbf{Z}$, that is,

$$
g(\delta(y)-v t(y))=\sum_{i=1}^{m} g_{i}\left(x_{i} \tilde{T}_{\underline{D}}^{s_{i}} x_{i}^{\prime} \tilde{T}_{\underline{D}}^{-s_{i}}-x_{i}^{\prime} \tilde{T}_{\underline{D}}^{1-s_{i}} x_{i} \tilde{T}_{\underline{D}}^{s_{i}-1}\right) .
$$

42.8. We show that for any $y, y^{\prime} \in H_{n}$ we have

(a) $\mathfrak{f} \eta \tilde{\omega}\left(y y^{\prime}-y^{\prime} \tilde{T}_{\underline{D}} y \tilde{T}_{\underline{D}}^{-1}\right)=0$.

Let $w \in \mathbf{W}, \lambda \in \underline{\mathfrak{s}}_{n}$. Let $\mathcal{L} \in \lambda$. If $w \underline{D} \lambda=\lambda$, using notation and results in 31.6, 31.7, we have

$$
\begin{aligned}
\mathfrak{f} \eta \tilde{\omega}\left(v^{l(w)} \tilde{T}_{w} 1_{\underline{D} \lambda}\right) & =\operatorname{gr}\left(K_{\mathbf{I}, D}^{w, \mathcal{L}}\right) \\
=\sum_{A} \chi_{v}^{A}\left(K_{\mathbf{I}, D}^{w, \mathcal{L}}\right) & =\sum_{A} \tilde{\zeta}^{A}\left(v^{l(w)} \tilde{T}_{w} 1_{\underline{D} \lambda} \tilde{T}_{\underline{D}}\right)=\sum_{A} \zeta^{A}\left(v^{l(w)} \tilde{T}_{w} 1_{\underline{D} \lambda} \tilde{T}_{\underline{D}}\right)
\end{aligned}
$$

(the last equation comes from $31.7($ e); $A$ runs over the objects in $\hat{D}$ up to isomorphism such that $\zeta^{A} \neq 0$.) The equation

$$
\mathfrak{f} \eta \tilde{\omega}\left(v^{l(w)} \tilde{T}_{w} 1_{\underline{D} \lambda}\right)=\sum_{A} \zeta^{A}\left(v^{l(w)} \tilde{T}_{w} 1_{\underline{D} \lambda} \tilde{T}_{\underline{D}}\right)
$$


holds also if $w \underline{D} \lambda \neq \lambda$ (in this case both sides are 0 ). It follows that

$$
\mathfrak{f} \eta \tilde{\omega}(x)=\sum_{A} \zeta^{A}\left(x \tilde{T}_{\underline{D}}\right) \text { for any } x \in H_{n} .
$$

We deduce

$$
\mathfrak{f} \eta \tilde{\omega}\left(y y^{\prime}-y^{\prime} \tilde{T}_{\underline{D}} y \tilde{T}_{\underline{D}}^{-1}\right)=\sum_{A}\left(\zeta^{A}\left(y y^{\prime} \tilde{T}_{\underline{D}}\right)-\zeta^{A}\left(y \epsilon(y) \tilde{T}_{\underline{D}}\right)\right)=0
$$

where the last equality follows from 31.8. This proves (a).

Proposition 42.9. Let $y \in H$. We have $\mathbf{d}(\mathfrak{f} \eta \tilde{\omega}(y))=\mathfrak{f} \eta \tilde{\omega}(\vartheta(y)) \in \mathfrak{K}(D)$ with $\mathbf{d}: \mathfrak{K}(D) \rightarrow \mathfrak{K}(\Delta)$ as in 42.2.

If $y \in H_{D}^{\prime}$ (see 42.3), both sides of the desired equality are 0 . (Note that $\vartheta$ maps $H_{D}$ into itself and $H_{D}^{\prime}$ into itself.) Hence we may assume that $y \in H_{D}$. We can assume that $y \in H_{n}$ where $n \in \mathbf{N}_{\mathbf{k}}^{*}$. Then $y \in H_{n, D}$. By 42.4 it is enough to show that $\mathfrak{f} \eta \tilde{\omega}(\delta(y)-\vartheta(y))=0$. Let $g, g_{i}, x_{i}, x_{i}^{\prime}, s_{i}$ be as in $42.7(\mathrm{f})$. Since $g \neq 0$, it is enough to show that $g \mathfrak{f} \eta \tilde{\omega}(\delta(y)-\vartheta(y))=0$ or that $\mathfrak{f} \eta \tilde{\omega}(g(\delta(y)-\vartheta(y)))=0$. Using 42.7 it is enough to show that

$$
\mathfrak{f} \eta \tilde{\omega}\left(\sum_{i=1}^{m} g_{i}\left(x_{i} \tilde{T}_{\underline{D}}^{s_{i}} x_{i}^{\prime} \tilde{T}_{\underline{D}}^{-s_{i}}-x_{i}^{\prime} \tilde{T}_{\underline{D}}^{1-s_{i}} x_{i} \tilde{T}_{\underline{D}}^{s_{i}-1}\right)=0 .\right.
$$

Hence it is enough to show that

$$
\mathfrak{f} \eta \tilde{\omega}\left(x \tilde{T}_{\underline{D}}^{s} x^{\prime} \tilde{T}_{\underline{D}}^{-s}-x^{\prime} \tilde{T}_{\underline{D}}^{1-s} x \tilde{T}_{\underline{D}}^{s-1}\right)=0
$$

for any $x, x^{\prime} \in H_{n}$ and any $s \in \mathbf{Z}$. We have

$$
x \tilde{T}_{\underline{D}}^{s} x^{\prime} \tilde{T}_{\underline{D}}^{-s}-x^{\prime} \tilde{T}_{\underline{D}}^{1-s} x \tilde{T}_{\underline{D}}^{s-1}=\left(z-\tilde{T}_{\underline{D}}^{-s} z \tilde{T}_{\underline{D}}^{s}\right)+\left(z^{\prime} x^{\prime}-x^{\prime} \tilde{T}_{\underline{D}} z^{\prime} \tilde{T}_{\underline{D}}^{-1}\right)
$$

where $z=x \tilde{T}_{\underline{D}}^{s} x^{\prime} \tilde{T}_{\underline{D}}^{-s} \in H_{n}$ and $z^{\prime}=\tilde{T}_{\underline{D}}^{-s} x \tilde{T}_{\underline{D}}^{s} \in H_{n}$. Hence it is enough to show that $\mathfrak{f} \eta \tilde{\omega}\left(z^{\prime} x^{\prime}-x^{\prime} \tilde{T}_{\underline{D}} z^{\prime} \tilde{T}_{D}^{-1}\right)=0$ (see $\left.42.8(\mathrm{a})\right)$ and

(a) $\mathfrak{f} \eta \tilde{\omega}\left(z-\tilde{T}_{\underline{D}}^{-s} z \tilde{T}_{\underline{D}}^{s}\right)=0$

for any $z \in H_{n}$. This follows from 41.6(c).

\section{REFERENCES}

[BBD] A. Beilinson, J. Bernstein, P. Deligne, Faisceaux pervers, Astérisque 100 (1982). MR 0751966 (86g:32015)

[De] P. Deligne, La conjecture de Weil, II, Publ.Math. I.H.E.S. 52 (1980), 137-252. MR0601520 (83c:14017)

[G] V. Ginzburg, Admissible modules on a symmetric space, Astérisque 173-174 (1989), 199255. MR1021512 (91c:22030)

[Gr] I. Grojnowski, Character sheaves on symmetric spaces, Ph.D. thesis, MIT, 1992.

[L3] G. Lusztig, Character sheaves, I, Adv. Math. 56 (1985), 193-237; II, vol. 57, 1985, pp. 226265; III, vol. 57, 1985 , pp. 266-315; IV, vol. 59 , 1986, pp. 1-63; V, vol. 61 , 1986, pp. 103155. MR0792706 (87b:20055) MR0806210 (87m:20118a) MR0825086 (87m:20118b) MR:0866163 (87m:20118d)

[L9] G. Lusztig, Character sheaves on disconnected groups, I, Represent. Theory. (electronic) 7 (2003), 374-403; II, vol. 8, 2004, pp. 72-124; III, vol. 8, 2004, pp. 125-144; IV, vol. 8, 2004, pp. 145-178; Errata, vol. 8, 2004, pp. 179-179; V, vol. 8, 2004, pp. 346-376; VI, vol. 8, 2004, pp. 377-413; VII, vol. 9, 2005, pp. 209-266; VIII, vol. 10, 2006. MR2017063(2006d:20090a) MR.2048588 (2006d:20090b) MR2048589|(2006d:20090c) MR:2048590||(2006d:20090d) MR 2077486 (2005h:20111) MR 2084488 (2005h:20112) MR 2133758 (2006e:20089)

[L14] G. Lusztig, Characters of reductive groups over a finite field, Ann. Math. Studies, vol. 107, Princeton Univ. Press, 1984. MR0742472 (86j:20038) 
[L10] G. Lusztig, Parabolic character sheaves, I, Moscow Math. J. 4 (2004), 153-179. MR2074987 (2006d:20091a)

[MV] I. Mirković, K. Vilonen, Characteristic varieties of character sheaves, Invent. Math. 93 (1988), 405-418. MR0948107 (89i:20066)

Department of Mathematics, Massachusetts Institute of Technology, Cambridge, MASSACHUSETTS 02139 